\title{
Ranking Functions for Linear-Constraint Loops
}

\author{
Amir M. Ben-Amram*1 and Samir Genaim ${ }^{\dagger 2}$ \\ ${ }^{1}$ The Academic College of Tel-Aviv Yaffo \\ ${ }^{2}$ Complutense University of Madrid
}

July 8,2013

\begin{abstract}
In this paper we study the complexity of the problems: given a loop, described by linear constraints over a finite set of variables, is there a linear or lexicographical-linear ranking function for this loop? While existence of such functions implies termination, these problems are not equivalent to termination. When the variables range over the rationals (or reals), it is known that both problems are PTIME decidable. However, when they range over the integers, whether for single-path or multipath loops, the complexity has not yet been determined. We show that both problems are coNP-complete. However, we point out some special cases of importance of PTIME complexity. We also present complete algorithms for synthesizing linear and lexicographical-linear ranking functions, both for the general case and the special PTIME cases. Moreover, in the rational setting, our algorithm for synthesizing lexicographical-linear ranking functions extends existing ones, because our class of ranking functions is more general, yet it has polynomial time complexity.
\end{abstract}

\section{Introduction}

Termination analysis has received a considerable attention and nowadays several powerful tools for the automatic termination analysis of different programming languages and computational models exist Albert et al. 2007; Cook et al. 2006, Giesl et al. 2004, Harris et al. 2011; Kroening et al. 2010 Spoto et al.|2010. Much of the recent development in termination analysis has benefited from techniques that deal with one loop at a time, where a loop is specified by a loop guard and a (noniterative) loop body.

Very often, these loops are abstracted so that the state of the program during the loop is represented by a finite set of integer variables, the loop guard is a conjunction of linear inequalities, and the body modifies the variables in an affine linear way, as in the following example:

$$
\text { while }\left(x_{2}-x_{1} \leq 0, x_{1}+x_{2} \geq 1\right) \text { do } x_{2}^{\prime}=x_{2}-2 x_{1}+1, x_{1}^{\prime}=x_{1}
$$

where tagged variables represent the values at the completion of an iteration. When the variables are modified in the loop body so that they are not affine linear functions of the old ones, the effect

*amirben@mta.ac.il

†samir.genaim@fdi.ucm.es 
is sometimes captured (or approximated) by means of linear constraints. E.g., the $\mathrm{C}$ language loop "while $(4 * \mathrm{x}>=\mathrm{y} \& \& \mathrm{y}>=1)$ do $\mathrm{x}=(2 * \mathrm{x}+1) / 5$;", which involves integer division, can be represented by linear constraints as follows (since $2 * x+1$ is always positive)

$$
\text { while }\left(4 x_{1} \geq x_{2}, x_{2} \geq 1\right) \text { do } 5 x_{1}^{\prime} \leq 2 x_{1}+1,5 x_{1}^{\prime} \geq 2 x_{1}-3, x_{2}^{\prime}=x_{2}
$$

Linear constraints might also be used to model changes to data structures, the variables representing a size abstraction such as length of lists, depth of trees, etc. [Bruynooghe et al. 2007, Lee et al. 2001; Lindenstrauss and Sagiv 1997; Spoto et al. 2010]. For a precise definition of the loop representations we consider, see Section 2 they also include multipath loops where alternative paths in the loop body are represented.

A standard technique to prove the termination of a loop is to find a ranking function. Such a function maps a program state (a valuation of the variables) into an element of some well-founded ordered set, such that the value descends (in the appropriate order) whenever the loop completes an iteration. Since descent in a well-founded set cannot be infinite, this proves that the loop must terminate. This definition of "ranking function" is very general; in practice, researchers have often limited themselves to a convenient and tractable form of ranking function, so that an algorithm to find the function - if there is one - might be found.

A frequently used class of ranking functions is based on affine linear functions. In this case, we seek a function $\rho\left(x_{1}, \ldots, x_{n}\right)=a_{1} x_{1}+\cdots+a_{n} x_{n}+a_{0}$, with the rationals as a co-domain, such that

(i) $\rho(\bar{x}) \geq 0$ for any valuation $\bar{x}$ that satisfies the loop guard; and

(ii) $\rho(\bar{x})-\rho\left(\bar{x}^{\prime}\right) \geq 1$ for any transition (single execution of the loop body) that starts in $\bar{x}$ and leads to $\bar{x}^{\prime}$.

This automatically induces the piecewise-linear ranking function: $f(\bar{x})=\rho(\bar{x})+1$ if $\bar{x}$ satisfies the loop guard and 0 otherwise, with the non-negative rationals as a co-domain but ordered w.r.t. $a \succeq b$ if and only if $b \geq a+1$ (which is well-founded). For simplicity, we call $\rho$ itself a linear ranking function instead of referring to $f$.

An algorithm to find a linear ranking function using linear programming $(L P)$ was found by multiple researchers in different places and times and in some alternative versions Alias et al. 2010; Colón and Sipma 2001; Feautrier 1992a; Mesnard and Serebrenik|2008; Podelski and Rybalchenko 2004: Sohn and Gelder 1991. Since $L P$ has a polynomial time complexity, most of these methods yield polynomialtime algorithms. Generally speaking, they are based on the fact that $L P$ can precisely decide whether a given inequality is implied by a set of other inequalities, and can even be used to generate any implied inequality. After all, conditions (ii) and (ii) above are inequalities that should be implied by the constraints that define the loop guard and body. This approach can, in a certain sense, be sound and complete.

Soundness means that it produces a correct linear ranking function, if it succeeds; completeness means that if a linear ranking function exists, it will succeed. In other words, there are no false negatives. A completeness claim appears in some of the references, and we found it cited several times. In our opinion, it has created a false impression that the Linear Ranking problem for linear-constraint loops with integer variables was completely solved (and happily classified as polynomial time).

The fly in the ointment is the fact that these solutions are only complete when the variables range over the rationals, which means that the linear ranking function has to fulfill its requirements for any rational valuation of the variables that satisfies the loop guard. But this may lead to a false negative if the variables are, in fact, integers. The reader may turn to the two loops above and note that both 
of them do not terminate over the rationals at all (for the first, consider $x_{1}=x_{2}=\frac{1}{2}$; for the second, $x_{1}=\frac{1}{4}$ and $x_{2}=1$ ). But they have linear ranking functions valid for all integer valuations, which we derive in Section 3.4

This observation has led us to investigate the Linear Ranking problem for single-path and multipath linear constraint loops. We present several fundamental new results on this problem. We have confirmed that this problem is indeed harder in the integer setting, proving it to be coNP-complete (as a decision problem), even for loops that only manipulate integers in a finite range. On a positive note, this shows that there is a complete solution, even if exponential-time. We give such a solution both to the decision problem and to the synthesis problem. The crux of the coNP-completeness proof, and the corresponding synthesis algorithm, is that we rely on the generator representation of the transition polyhedron defined by the loop constraints. We provide sufficient and necessary conditions for the existence of a linear ranking function that use the vertices and rays of this representation.

Another positive news for the practically-minded reader is that some special cases of importance do have a PTIME solution, because they reduce (with no effort, or with a polynomial-time computation) to the rational case. We present several such cases, which include, among others, loops in which the body is a sequence of linear affine updates with integer coefficients, as in loop (1) above, and the condition is defined by either an extended form of difference constraints, a restricted form of Two Variables Per Inequality constraints, or a cone (constraints where the free constant is zero). Some cases in which the body involves linear constraints are also presented.

But linear ranking functions do not suffice for all loops, and, in particular for multipath loops, lexicographic-linear ranking functions are a natural extension. Such functions are a tuple of affine functions, such that in every iteration of the loop, the value of the tuple decreases lexicographically. Such a function will work, for example, for the following multipath loop

$$
\text { loop: }\left\{x_{1} \geq 0, x_{2} \geq 0, x_{1}^{\prime}=x_{1}-1\right\} \vee\left\{x_{1} \geq 0, x_{2} \geq 0, x_{2}^{\prime}=x_{2}-1, x_{1}^{\prime}=x_{1}\right\}
$$

where in the first path $x_{1}$ decreases towards zero and $x_{2}$ is changed unpredictably, while in the second path $x_{2}$ decreases towards zero and $x_{1}$ is unchanged. Clearly, $\left\langle x_{1}, x_{2}\right\rangle$ always decreases lexicographically, but there can be no single linear ranking function for this loop.

In Section 5 we analyze the complexity of the the decision problem: is there a lexicographic-linear ranking function for a given loop? We also give a complete synthesis algorithm. Our point of departure (corresponding to the case of linear functions) is the known polynomial-time algorithm of Alias et al. [2010], based on $L P$, that is claimed to be complete - and as explained above, is only complete when one extends the domain of the variables to the rationals. We show that the corresponding decision problem is, like the case of linear ranking function, coNP-complete when the variables are restricted to hold integers. We also give a novel complete synthesis algorithm. The algorithm is of exponential time complexity, but becomes polynomial-time in special cases corresponding to those identified in the context of linear ranking functions.

We also consider the application of the algorithm to the setting of rational data; in this setting it has polynomial time complexity and extends the one of Alias et al. [2010], because our class of ranking functions is more general. The algorithm produces a function that descends lexicographically in the rationals; for example, if it produces $\left\langle x_{1}, x_{2}\right\rangle$, it ensures that in every possible transition either $x_{1}>x_{1}^{\prime}$ and $x_{1} \geq 0$ or $x_{1}=x_{1}^{\prime}$ and $x_{2}>x_{2}^{\prime}$ and $x_{2} \geq 0$. If one is only interested in integer data, such a function proves termination, and this relaxation to the rationals is therefore sound. Over the rationals, however, this lexicographic order is not well-founded - simply because the order $(>)$ on $\mathbb{Q}_{+}$ is not (consider the sequence $x_{1}=\frac{1}{2}, \frac{1}{3}, \frac{1}{4}, \ldots$ ). Interestingly, we prove that a function that descends in the lexicographic extension of the order $(>)$ can always be turned into one that descends in the 
lexicographic extension of the order $a \succeq b$ (defined as $b \geq a+1$ ), and therefore implies termination.

We prove some properties of our synthesis algorithm, for example that the dimension (the length of the tuple) of the functions it produces is always the smallest possible.

Our results should be of interest to all users of ranking functions, and in fact their uses go beyond termination proofs. For example, they have been used to provide an upper bound on the number of iterations of a loop in program complexity analysis Albert et al. 2011, Alias et al. 2010 and to automatically parallelize computations Darte 2010; Feautrier 1992a]. We remark that in termination analysis, the distinction between integers and rationals has already been considered, both regarding ranking-function generation [Bradley et al. 2005b; Cook et al. 2010; Feautrier 1992a and the very decidability of the termination problem [Ben-Amram et al. |2012: Braverman $|2006|$ Tiwari |2004]. All these works left the integer case open. Interestingly, our results provide an insight on how to make the solution proposed by Bradley et al. 2005b, for synthesizing linear ranking functions, complete (see Section 7).

Our tool iRANKFINDER implements the algorithms mentioned above (and more) and can be tried out online (see Section 6).

This paper is organized as follows. Section 2 gives definitions and background information regarding linear-constraint loops, linear and lexicographic-linear ranking functions, and the mathematical notions involved. Section 3 proves that the decision problem "is there a linear ranking function for an integer loop", is coNP-complete, and also presents an exponential-time ranking-function synthesis algorithm. Section 4 discusses PTIME-solvable cases. Section 5 studies the complexity of the decision problem "is there a lexicographic-linear ranking function for a given loop", both for integer and rational data, and proves that it is coNP-complete and PTIME respectively. It also develops corresponding complete synthesis algorithms. Section 6 describes a prototype implementation. Section 7 surveys related previous work. Section 8 concludes. A conference version of this paper, including the results on linear ranking functions (but not lexicographic-linear ranking functions), has been presented at POPL 2013 Ben-Amram and Genaim 2013.

\section{Preliminaries}

In this section we recall some results on (integer) polyhedra on which we will rely along the paper, define the kind of loops we are interested in, and formally define the linear and lexicographic-linear ranking function problems for such loops.

\subsection{Integer Polyhedra}

We recall some useful definitions and properties, all following Schrijver [1986].

Polyhedra. A rational convex polyhedron $\mathcal{P} \subseteq \mathbb{Q}^{n}$ (polyhedron for short) is the set of solutions of a set of inequalities $A \mathbf{x} \leq \mathbf{b}$, namely $\mathcal{P}=\left\{\mathbf{x} \in \mathbb{Q}^{n} \mid A \mathbf{x} \leq \mathbf{b}\right\}$, where $A \in \mathbb{Q}^{m \times n}$ is a rational matrix of $n$ columns and $m$ rows, $\mathbf{x} \in \mathbb{Q}^{n}$ and $\mathbf{b} \in \mathbb{Q}^{m}$ are column vectors of $n$ and $m$ rational values respectively. We say that $\mathcal{P}$ is specified by $A \mathbf{x} \leq \mathbf{b}$. We use calligraphic letters, such as $\mathcal{P}$ and $\mathcal{Q}$ to denote polyhedra. The set of recession directions of a polyhedron $\mathcal{P}$ specified by $A \mathbf{x} \leq \mathbf{b}$ is the set $\mathcal{R}_{\mathcal{P}}=\left\{\mathbf{y} \in \mathbb{Q}^{n} \mid A \mathbf{y} \leq \mathbf{0}\right\}$. 
Integer Polyhedra. For a given polyhedron $\mathcal{P} \subseteq \mathbb{Q}^{n}$ we let $I(\mathcal{P})$ be $\mathcal{P} \cap \mathbb{Z}^{n}$, i.e., the set of integer points of $\mathcal{P}$. The integer hull of $\mathcal{P}$, commonly denoted by $\mathcal{P}_{I}$, is defined as the convex hull of $I(\mathcal{P})$, i.e., every rational point of $\mathcal{P}_{I}$ is a convex combination of integer points. This property is fundamental to our results. It is known that $\mathcal{P}_{I}$ is also a polyhedron. An integer polyhedron is a polyhedron $\mathcal{P}$ such that $\mathcal{P}=\mathcal{P}_{I}$. We also say that $\mathcal{P}$ is integral.

Generator representation. Polyhedra also have a generator representation in terms of vertices and rays ${ }^{1}$, written as

$$
\mathcal{P}=\operatorname{convhull}\left\{\mathbf{x}_{1}, \ldots, \mathbf{x}_{m}\right\}+\operatorname{cone}\left\{\mathbf{y}_{1}, \ldots, \mathbf{y}_{t}\right\} .
$$

This means that $\mathbf{x} \in \mathcal{P}$ if and only if $\mathbf{x}=\sum_{i=1}^{m} a_{i} \cdot \mathbf{x}_{i}+\sum_{j=1}^{t} b_{j} \cdot \mathbf{y}_{j}$ for some rationals $a_{i}, b_{j} \geq 0$, where $\sum_{i=1}^{m} a_{i}=1$. Note that $\mathbf{y}_{1}, \ldots, \mathbf{y}_{t}$ are the recession directions of $\mathcal{P}$, i.e., $\mathbf{y} \in \mathcal{R}_{\mathcal{P}}$ if and only if $\mathbf{y}=\sum_{j=1}^{t} b_{j} \cdot \mathbf{y}_{j}$ for some rational $b_{j} \geq 0$. If $\mathcal{P}$ is integral, then there is a generator representation in which all $\mathbf{x}_{i}$ and $\mathbf{y}_{j}$ are integer. An empty polyhedron is represented by an empty set of vertices and rays.

Faces. If $\mathbf{c}$ is a nonzero vector and $a=\max \{\mathbf{c} \cdot \mathbf{x} \mid \mathbf{x} \in \mathcal{P}\}$, then $\mathcal{H}=\left\{\mathbf{x} \in \mathbb{Q}^{n} \mid \mathbf{c} \cdot \mathbf{x}=a\right\}$ is called a supporting hyperplane for $\mathcal{P}$. A non-empty subset $\mathcal{F} \subseteq \mathcal{P}$ is called a face if $\mathcal{F}=\mathcal{P}$ or $\mathcal{F}$ is an intersection of $\mathcal{P}$ with a supporting hyperplane Schrijver 1986, p. 101]. In the latter case we say that $\mathcal{F}$ is a proper face of $\mathcal{P}$. Alternatively, $\mathcal{F}$ is face of $\mathcal{P}$ if and only if it can be obtained by turning some inequalities of $A \mathrm{x} \leq b$ to equalities [Schrijver 1986. Sec. 16.3, p. 231]. It is known that a polyhedron $\mathcal{P}$ is integral if and only if every face of $\mathcal{P}$ includes an integer point [Schrijver 1986, Sec. 16.3, p. 231]. This implies that the faces of an integral polyhedron $\mathcal{P}$ are integral.

Dimension of polyhedra. Let $A^{=} \mathbf{x} \leq \mathbf{b}^{=}$be the set of all implicit equalities in $A \mathbf{x} \leq \mathbf{b}\left(\mathbf{a}_{i} \cdot \mathbf{x} \leq \mathbf{b}_{i}\right.$ is an implicit inequality if $\mathbf{a}_{i} \cdot \mathbf{x}=\mathbf{b}_{i}$ holds for any $\mathbf{x} \in \mathcal{P}$ ). The affine hull of $\mathcal{P}$ is defined as aff.hull $(\mathcal{P})=\left\{\mathbf{x} \in \mathbb{Q}^{n} \mid A^{=} \mathbf{x}=\mathbf{b}^{=}\right\}$. The dimension of the affine hull is the dimension of the linear subspace $\left\{\mathbf{x} \mid A^{=} \mathbf{x}=\mathbf{0}\right\}$ (i.e, the cardinality of the bases). Alternatively, it is equal to $n$ minus the rank of the matrix $A^{=}$. The dimension of a polyhedron $\mathcal{P} \subseteq \mathbb{Q}^{n}$, denoted by $\operatorname{dim}(\mathcal{P})$, is equal to the dimension of its affine hull. The dimension of the empty polyhedron, by convention, is -1 . The dimension of a proper face of $\mathcal{P}$ is at least 1 less than that of $\mathcal{P}$. Note that when $\operatorname{dim}(\mathcal{P})=0$ then $\mathcal{P}$ is a single point.

Relative interior. The relative interior of $\mathcal{P}$ is defined as $\mathrm{ri}(\mathcal{P})=\{\mathbf{x} \mid \exists \epsilon>0 . B(\mathbf{x}, \epsilon) \cap$ aff.hull $(\mathcal{P}) \subseteq$ $\mathcal{P}\}$ where $B(\mathbf{x}, \epsilon)$ is a ball of radius $\epsilon$ centered on $\mathbf{x}$. Intuitively, it is the set of all points which are not on the "edge" of $\mathcal{P}$. Note that $\mathrm{x} \in \operatorname{ri}(\mathcal{P})$ if and only if $\mathrm{x} \in \mathcal{P}$ and $\mathbf{x}$ does not belong to any proper face of $\mathcal{P}$. When $\operatorname{dim}(\mathcal{P})=0$, the single point of $\mathcal{P}$ is in the relative interior (since $\mathcal{P}$ does not have any proper face).

Size of polyhedra. Complexity of algorithms on polyhedra is measured in this paper by running time, on a conventional computational model (polynomially equivalent to a Turing machine), as a function of the bit-size of the input. Following Schrijver 1986, Sec. 2.1], we define the bit-size of an integer $x$ as $\|x\|=1+\lceil\log (|x|+1)\rceil$; the bit-size of an $n$-dimensional vector a as $\|\mathbf{a}\|=n+\sum_{i=1}^{n}\left\|a_{i}\right\|$; and the bit-size of an inequality $\mathbf{a} \cdot \mathbf{x} \leq c$ as $1+\|c\|+\|\mathbf{a}\|$. For a polyhedron $\mathcal{P} \subseteq \mathbb{Q}^{n}$ defined by $A \mathbf{x} \leq \mathbf{b}$,

\footnotetext{
${ }^{1}$ Technically, the $\mathbf{x}_{1}, \ldots, \mathbf{x}_{n}$ are only vertices if the polyhedron is pointed.
} 
we let $\|\mathcal{P}\|_{b}$ be the bit-size of $A \mathbf{x} \leq \mathbf{b}$, which we can take as the sum of the sizes of the inequalities. The facet size, denoted by $\|\mathcal{P}\|_{f}$, is the smallest number $\phi \geq n$ such that $\mathcal{P}$ may be described by some $A \mathbf{x} \leq \mathbf{b}$ where each inequality in $A \mathbf{x} \leq \mathbf{b}$ fits in $\phi$ bits. Clearly, $\|\mathcal{P}\|_{f} \leq\|\mathcal{P}\|_{b}$. The vertex size, denoted by $\|\mathcal{P}\|_{v}$, is the smallest number $\psi \geq n$ such that $\mathcal{P}$ has a generator representation in which each of $\mathbf{x}_{i}$ and $\mathbf{y}_{j}$ fits in $\psi$ bits (the size of a vector is calculated as above). For integer polyhedra, we restrict the generators to be integer. The following theorems state some relations between the different bit-sizes defined above, they are later used to polynomially bound the bit-size of some set of integer points of $\mathcal{P}_{I}$. Both follows Schrijver 1986. Cor. 17.1a,17.1b, p. 238, and, Cor. 17.1a,17.1b, p. 238], who cites Karp and Papadimitriou [1980].

THEOREM 2.1. Let $\mathcal{P}$ be a rational polyhedron in $\mathbb{Q}^{n} ;$ then $\|\mathcal{P}\|_{v} \leq 4 n^{2}\|\mathcal{P}\|_{f}$ and $\|\mathcal{P}\|_{f} \leq 4 n^{2}\|\mathcal{P}\|_{v}$.

THEOREM 2.2. Let $\mathcal{P}$ be a rational polyhedron in $\mathbb{Q}^{n}$; then $\left\|\mathcal{P}_{I}\right\|_{v} \leq 6 n^{3}\|\mathcal{P}\|_{f}$ and $\left\|\mathcal{P}_{I}\right\|_{f} \leq$ $24 n^{5}\|\mathcal{P}\|_{f}$.

\subsection{Multipath linear-constraint loops}

A single-path linear-constraint loop ( $S L C$ for short) over $n$ variables $x_{1}, \ldots, x_{n}$ has the form

$$
\text { while }(B \mathbf{x} \leq \mathbf{b}) \text { do } A\left(\begin{array}{l}
\mathbf{x} \\
\mathbf{x}^{\prime}
\end{array}\right) \leq \mathbf{c}
$$

where $\mathbf{x}=\left(x_{1}, \ldots, x_{n}\right)^{\mathrm{T}}$ and $\mathbf{x}^{\prime}=\left(x_{1}^{\prime}, \ldots, x_{n}^{\prime}\right)^{\mathrm{T}}$ are column vectors, and for some $p, q>0, B \in \mathbb{Q}^{p \times n}$, $A \in \mathbb{Q}^{q \times 2 n}, \mathbf{b} \in \mathbb{Q}^{p}, \mathbf{c} \in \mathbb{Q}^{q}$. The constraint $B \mathbf{x} \leq \mathbf{b}$ is called the loop condition (a.k.a. the loop guard) and the other constraint is called the update. The update is called deterministic if, for a given $\mathbf{x}$ (satisfying the loop condition) there is at most one $\mathbf{x}^{\prime}$ satisfying the update constraint. The update is called linear if it can be rewritten as

$$
\mathbf{x}^{\prime}=A^{\prime} \mathbf{x}+\mathbf{c}^{\prime}
$$

for a matrix $A^{\prime}$ and vector $\mathbf{c}^{\prime}$ of appropriate dimensions. We say that the loop is a rational loop if $\mathbf{x}$ and $\mathbf{x}^{\prime}$ range over $\mathbb{Q}^{n}$, and that it is an integer loop if they range over $\mathbb{Z}^{n}$.

We say that there is a transition from a state $\mathbf{x} \in \mathbb{Q}^{n}$ to a state $\mathbf{x}^{\prime} \in \mathbb{Q}^{n}$, if $\mathbf{x}$ satisfies the condition and $\mathbf{x}$ and $\mathbf{x}^{\prime}$ satisfy the update. A transition can be seen as a point $\left(\begin{array}{c}\mathbf{x} \\ \mathbf{x}^{\prime}\end{array}\right) \in \mathbb{Q}^{2 n}$, where its first $n$ components correspond to $\mathbf{x}$ and its last $n$ components to $\mathbf{x}^{\prime}$. For ease of notation, we denote $\left(\begin{array}{l}\mathbf{x} \\ \mathbf{x}^{\prime}\end{array}\right)$ by $\mathrm{x}^{\prime \prime}$. The set of all transitions $\mathbf{x}^{\prime \prime} \in \mathbb{Q}^{2 n}$ will be denoted, as a rule, by $\mathcal{Q}$. The transition polyhedron $\mathcal{Q}$ is specified by the set of inequalities $A^{\prime \prime} \mathrm{x}^{\prime \prime} \leq \mathbf{c}^{\prime \prime}$ where

$$
A^{\prime \prime}=\left(\begin{array}{cc}
B & 0 \\
A
\end{array}\right) \quad \mathbf{c}^{\prime \prime}=\left(\begin{array}{l}
\mathbf{b} \\
\mathbf{c}
\end{array}\right)
$$

Note that we may assume that $\mathcal{Q}$ does not include the origin, for if it includes it, the loop is clearly non-terminating (this condition is easy to check). Hence, $\mathcal{Q}$ is not a cone (i.e., $m \geq 1$ in the generator representation). The polyhedron defined by the loop condition $B \mathbf{x} \leq \mathbf{b}$ will be denoted by $\mathcal{C}$ and referred to as the condition polyhedron.

A multipath linear-constraint loop ( $M L C$ for short) differs by having alternative loop conditions and updates, which are, in principle, chosen non-deterministically (though the constraints may enforce a deterministic choice):

$$
\text { loop } \bigvee_{i=1}^{k}\left[B_{i} \mathbf{x} \leq \mathbf{b}_{i} \wedge A_{i}\left(\begin{array}{l}
\mathbf{x} \\
\mathbf{x}^{\prime}
\end{array}\right) \leq \mathbf{c}_{i}\right]
$$


This means that the $i$-th update can be applied if the $i$-th condition is satisfied. Following the notation of $S L C$ loops, the transitions of an $M L C$ loop are specified by the transition polyhedra $\mathcal{Q}_{1}, \ldots, \mathcal{Q}_{k}$, where each $\mathcal{Q}_{i}$ is specified by $A_{i}^{\prime \prime} \mathbf{x}^{\prime \prime} \leq \mathbf{c}_{i}^{\prime \prime}$. The polyhedron defined by the condition $B_{i} \mathbf{x} \leq \mathbf{b}_{i}$ is denoted by $\mathcal{C}_{i}$.

For simplifying the presentation, often we write loops with explicit equalities and inequalities instead of the matrix representation. We also might refer to loops by their corresponding transition polyhedra, or the sets of inequalities that define these polyhedra.

\subsection{Linear Ranking Functions}

A linear function $\rho: \mathbb{Q}^{n} \rightarrow \mathbb{Q}$ is of the form $\rho(\mathbf{x})=\vec{\lambda} \cdot \mathbf{x}+\lambda_{0}$ where $\vec{\lambda} \in \mathbb{Q}^{n}$ is a row vector and $\lambda_{0} \in \mathbb{Q}$. For ease of notation we sometimes refer to a linear function using the row vector $\left(\lambda_{0}, \vec{\lambda}\right) \in \mathbb{Q}^{n+1}$. For a given function $\rho$, we define the function $\Delta \rho: \mathbb{Q}^{2 n} \mapsto \mathbb{Q}$ as $\Delta \rho\left(\mathbf{x}^{\prime \prime}\right)=\rho(\mathbf{x})-\rho\left(\mathbf{x}^{\prime}\right)$. Next we define when a linear function is a linear ranking function ( $L R F$ for short) for a given rational or integer $M L C$ loop.

Definition 2.3. Given a set $T \subseteq \mathbb{Q}^{2 n}$, representing transitions, we say that $\rho$ is a $L R F$ for $T$ if the following hold for every $\mathbf{x}^{\prime \prime} \in T$ :

$$
\begin{aligned}
\rho(\mathbf{x}) & \geq 0, \\
\Delta \rho\left(\mathbf{x}^{\prime \prime}\right) & \geq 1 .
\end{aligned}
$$

We say that $\rho$ is a $L R F$ for a rational loop, specified by $\mathcal{Q}_{1}, \ldots, \mathcal{Q}_{k}$, when it is a $L R F$ for all of $\mathcal{Q}_{1}, \ldots, \mathcal{Q}_{k}$ (equivalently, it is a $L R F$ for $\bigcup_{i=1}^{k} \mathcal{Q}_{i}$ ). We say that $\rho$ is a $L R F$ for an integer loop, specified by $\mathcal{Q}_{1}, \ldots, \mathcal{Q}_{k}$ polyhedra, when it is a $L R F$ for all of $I\left(\mathcal{Q}_{1}\right), \ldots, I\left(\mathcal{Q}_{k}\right)$.

Clearly, the existence of a $L R F$ implies termination of the loop. Note that in 8 ) we require $\rho$ to decrease at least by 1, whereas in the literature Podelski and Rybalchenko 2004 this 1 is sometimes replaced by $\delta>0$. It is easy to verify that these definitions are equivalent as far as the existence of a $L R F$ is concerned.

Definition 2.4. The decision problem Existence of a $L R F$ is defined by

Instance: an $M L C$ loop.

Question: does there exist a $L R F$ for this loop?

The decision problem is denoted by $\operatorname{LinRF}(\mathbb{Q})$ and $\operatorname{LinRF}(\mathbb{Z})$ for rational and integer loops respectively.

It is known that $\operatorname{LinRF}(\mathbb{Q})$ is PTIME-decidable Mesnard and Serebrenik 2008; Podelski and Rybalchenko 2004. In this paper, we focus on $\operatorname{LinRF}(\mathbb{Z})$.

\subsection{Lexicographic-Linear Ranking Functions}

A $d$-dimensional affine function $\tau: \mathbb{Q}^{n} \rightarrow \mathbb{Q}^{d}$ is a function of the form $\tau=\left\langle\rho_{1}, \ldots, \rho_{d}\right\rangle$, where each component $\rho_{i}: \mathbb{Q}^{n} \rightarrow \mathbb{Q}$ is an affine function. The number $d$ is informally called the dimension of the function (technically, it is the dimension of the co-domain). Next we define when a $d$-dimensional affine function is a lexicographic-linear ranking function ( $L L R F$ for short) for a given rational or integer $M L C$ loop. 
Definition 2.5. Let $T \subseteq \mathbb{Q}^{2 n}$ be a given set, representing transitions, and $\tau=\left\langle\rho_{1}, \ldots, \rho_{d}\right\rangle$ a $d$ dimensional affine function. We say that $\tau$ is a $L L R F$ for $T$ if and only if for every $\mathbf{x}^{\prime \prime} \in T$ there exists $i \leq d$ such that the following hold

$$
\begin{aligned}
\forall j<i . \Delta \rho_{j}\left(\mathbf{x}^{\prime \prime}\right) & \geq 0, \\
\forall j \leq i . \quad \rho_{j}(\mathbf{x}) & \geq 0, \\
\Delta \rho_{i}\left(\mathbf{x}^{\prime \prime}\right) & \geq 1 .
\end{aligned}
$$

We say that $\mathbf{x}^{\prime \prime}$ is ranked by $\rho_{i}$.

As for $L R F s$, we say that $\tau$ is a $L L R F$ for a rational loop $\mathcal{Q}_{1}, \ldots, \mathcal{Q}_{k}$ when it is a $L L R F$ for $\bigcup_{i=1}^{k} \mathcal{Q}_{i}$, and that it is a $L L R F$ for the corresponding integer loop if it is a $L L R F$ for $\bigcup_{i=1}^{k} I\left(\mathcal{Q}_{i}\right)$.

Note that in (11) we require $\rho_{i}$ to decrease at least by 1 . As for the case of $L R F s$, this 1 can be replaced by any $\delta_{i}>0$. It is easy to verify that these definitions are equivalent as far as the existence of a $L L R F$ is concerned. The existence of a $L L R F$ implies termination of the loop. This may be justified by converting the function into one that decreases in a well-founded set; such a function is

$$
\hat{\tau}\left(\mathbf{x}^{\prime \prime}\right)=\left\langle\max \left(0, \rho_{1}\left(\mathbf{x}^{\prime \prime}\right)\right), \ldots, \max \left(0, \rho_{d}\left(\mathbf{x}^{\prime \prime}\right)\right)\right\rangle,
$$

whose co-domain is $\left\langle\mathbb{Q}_{+}^{d}, \preceq_{l e x}\right\rangle$, where $\preceq_{l e x}$ is the lexicographic extension of the well-founded order: $a \preceq b$ iff $a+1 \leq b$.

Our class of $L L R F$ s differs somewhat from other classes of "lexicographic-linear ranking functions" that appeared in the literature Alias et al. 2010 Bradley et al. 2005a. Specifically, the definition in Alias et al. 2010 is more restrictive since it requires (10) to hold for all $1 \leq j \leq d$. The following example illustrates the difference.

EXAMPLE 2.6. Consider the $S L C$ loop

$$
\text { while }\left(x_{1} \geq 0, x_{2} \geq 0, x_{3} \geq-x_{1}\right) \text { do } x_{2}^{\prime}=x_{2}-x_{1}, x_{3}^{\prime}=x_{3}+x_{1}-2 .
$$

It has a $L L R F \tau=\left\langle x_{2}, x_{3}\right\rangle$ as in Definition 2.5 (over both rationals and integers), however, it does not have a $L L R F$ according to Alias et al. 2010]. Indeed, when $x_{2}$ decreases $x_{3}$ can be negative (e.g., for $x_{1}=1, x_{2}=2$ and $x_{3}=-1$ ).

Another difference from Alias et al. 2010] lies in the fact that they require the non-negativity conditions 10 to be implied by the loop guard. That is, it is not possible to use the constraints in the update part of the loop in proving this condition, when according to our definition it is possible.

The definition of Bradley et al. 2005a requires (10) to hold only for $j=i$, which adds flexibility, as we show next.

EXAMPLE 2.7. Consider the $M L C$ loop

$$
\text { loop : }\left\{x \geq 0, x^{\prime}=x-1\right\} \vee\left\{y \geq 0, y^{\prime}=y-1, x^{\prime} \leq x\right\} .
$$

It has a $L L R F \tau=\langle x, y\rangle$ according to the definition of Bradley et al. 2005a], however, it does not have one that satisfies Definition 2.5. Indeed, in transitions where $y$ decreases $x$ may be negative, but $x$ must be the first component.

Another difference is that Bradley et al. 2005a require a fixed association of ranking-function components with the paths of the loop. So, for example, they cannot have a 2-dimensional LLRF for an $S L C$ loop, as in Example 2.6 . 
Definition 2.8. The decision problem Existence of a $L L R F$ is defined by

Instance: an $M L C$ loop.

Question: does there exist a $L L R F$ for this loop?

The decision problem is denoted by $\operatorname{LexLinRF}(\mathbb{Q})$ and $\operatorname{LexLinRF}(\mathbb{Z})$ for rational and integer loops respectively.

\section{$3 \quad \operatorname{LinRF}(\mathbb{Z})$ is coNP-complete}

In this section we show that the $\operatorname{LiNRF}(\mathbb{Z})$ problem is coNP-complete; it is coNP-hard already for $S L C$ loops that restrict the variables to a finite range. We also show that $L R F s$ can be synthesized in deterministic exponential time.

This section is organized as follows: in Section 3.1 we show that $\operatorname{LinRF}(\mathbb{Z})$ is coNP-hard; in Section 3.2 we show that it is in coNP for $S L C$ loops, and in Section 3.3 for $M L C$ loops; finally, in Section 3.4 we describe an algorithm for synthesizing LRFs.

\section{1 coNP-hardness}

We prove coNP-hardness in a strong form. Recall that a number problem (a problem whose instance is a matrix of integers) Prob is strongly hard for a complexity class, if there are polynomial reductions from all problems in that class to Prob such that the values of all numbers created by the reduction are polynomially bounded by the input bit-size. Assuming NP $\neq \mathrm{P}$, strongly NP-hard (or coNP-hard) problems cannot even have pseudo-polynomial algorithms Garey and Johnson 1979.

THEOREM 3.1. The $\operatorname{LinRF}(\mathbb{Z})$ problem is strongly coNP-hard, even for deterministic SLC loops.

Proof. The problem of deciding whether a polyhedron given by $B \mathbf{x} \leq \mathbf{b}$ contains no integer point is a well-known coNP-hard problem (an easy reduction from SAT Karp 1972]). We reduce this problem to $\operatorname{LinRF}(\mathbb{Z})$.

Given $B \in \mathbb{Z}^{m \times n}$ and $\mathbf{b} \in \mathbb{Z}^{m}$, we construct the following integer $S L C$ loop

$$
\text { while }\left(\begin{array}{cc}
B & -I \\
0 & -I
\end{array}\right)\left(\begin{array}{l}
\mathbf{x} \\
\mathbf{z}
\end{array}\right) \leq\left(\begin{array}{l}
\mathbf{b} \\
\mathbf{0}
\end{array}\right) \text { do }\left(\begin{array}{l}
\mathbf{x}^{\prime} \\
\mathbf{z}^{\prime}
\end{array}\right)=\left(\begin{array}{c}
\mathbf{x} \\
\mathbf{0}
\end{array}\right)
$$

where $\mathbf{x}=\left(x_{1}, \ldots, x_{n}\right)^{\mathrm{T}}, \mathbf{z}=\left(z_{1}, \ldots, z_{m}\right)^{\mathrm{T}}$ are integer variables, and $I$ is an identity matrix of size $m \times m$.

Suppose $B \mathbf{x} \leq \mathbf{b}$ has an integer solution $\mathbf{x}$. Then, it is easy to see that the loop does not terminate when starting from this $\mathbf{x}$ and $\mathbf{z}$ set to $\mathbf{0}$, since the guard is satisfied and the update does not change the values. Thus, it does not have any ranking function, let along a $L R F$.

Next, suppose $B \mathbf{x} \leq \mathbf{b}$ does not have an integer solution. Then, for any initial state for which the loop guard is enabled it must hold that $z_{1}+\cdots+z_{m}>0$, for otherwise $z_{1}, \ldots, z_{m}$ must be 0 in which case the constraint $B \mathbf{x}-I \mathbf{z} \leq \mathbf{b}$ has no integer solution. Since the updated vector $\mathbf{z}^{\prime}$ is deterministically set to $\mathbf{0}$, the guard will not be enabled in the next state, hence the loop terminates after one iteration. Clearly $z_{1}+\cdots+z_{m}>z_{1}^{\prime}+\cdots+z_{m}^{\prime}=0$, so we conclude that $z_{1}+\cdots+z_{m}$ is a $L R F$.

Note that in the above reduction we rely on the hardness of whether a given polyhedron is empty. This problem is coNP-hard even for bounded polyhedra (due to the reduction from SAT in which 
variables are bounded by 0 and 1). This means that even for loops that only manipulate integers in a rather small range, the problem is coNP-hard. The parameter "responsible" for the exponential behavior in this case is the number of variables.

\subsection{Inclusion in coNP for $S L C$ loops}

To prove that $\operatorname{LinRF}(\mathbb{Z})$ is in coNP, we show that the complement of $\operatorname{LinRF}(\mathbb{Z})$, the problem of nonexistence of a $L R F$, is in NP, that is, has a polynomially-checkable witness. In what follows we assume as input an $S L C$ loop with a transition polyhedron $\mathcal{Q} \subseteq \mathbb{Q}^{2 n}$. The input is given as the set of linear inequalities $A^{\prime \prime} \mathbf{x}^{\prime \prime} \leq \mathbf{c}^{\prime \prime}$ that define $\mathcal{Q}$. The proof follows the following lines:

1. We show that there is no $L R F$ for $I(\mathcal{Q})$ if and only if there is a witness that consists of two sets of integer points $X \subseteq I(\mathcal{Q})$ and $Y \subseteq I\left(\mathcal{R}_{\mathcal{Q}}\right)$, such that a certain set of inequalities $\Psi_{W S}(X, Y)$ has no solution over the rationals; and

2. We show that if there is a witness then there is one with bit-size polynomial in the input bit-size.

To make sense of the following definitions, think of a vector $\left(\lambda_{0}, \vec{\lambda}\right) \in \mathbb{Q}^{n+1}$ as a "candidate $L R F$ " that we may want to verify (or, in our case, to eliminate).

Definition 3.2. We say that $\mathbf{x}^{\prime \prime} \in I(\mathcal{Q})$ is a witness against $\left(\lambda_{0}, \vec{\lambda}\right) \in \mathbb{Q}^{n+1}$ if it fails to satisfy at least one of

$$
\begin{array}{r}
\vec{\lambda} \cdot \mathbf{x}+\lambda_{0} \geq 0 \\
\vec{\lambda} \cdot\left(\mathbf{x}-\mathbf{x}^{\prime}\right) \geq 1
\end{array}
$$

The set of $\left(\lambda_{0}, \vec{\lambda}\right)$ witnessed against by $\mathbf{x}^{\prime \prime}$ is denoted by $W\left(\mathbf{x}^{\prime \prime}\right)$.

Note that conditions $14 \mid 15)$ are obtained from 78 by writing $\rho$ explicitly.

Definition 3.3. We say that $\mathbf{y}^{\prime \prime} \in I\left(\mathcal{R}_{\mathcal{Q}}\right)$ is a homogeneous (component of a) witness (h-witness) against $\left(\lambda_{0}, \vec{\lambda}\right) \in \mathbb{Q}^{n+1}$ if it fails to satisfy at least one of

$$
\begin{aligned}
\vec{\lambda} \cdot \mathbf{y} & \geq 0 \\
\vec{\lambda} \cdot\left(\mathbf{y}-\mathbf{y}^{\prime}\right) & \geq 0
\end{aligned}
$$

The set of $\left(\lambda_{0}, \vec{\lambda}\right)$ h-witnessed against by $\mathbf{y}^{\prime \prime}$ is denoted by $W_{H}\left(\mathbf{y}^{\prime \prime}\right)$.

The meaning of the witness of Definition 3.2 is quite straightforward. Let us intuitively explain the meaning of an h-witness. Suppose that $\mathbf{x}^{\prime \prime}$ is a point in $\mathcal{Q}_{I}$, and $\mathbf{y}^{\prime \prime}$ is a ray of $\mathcal{Q}_{I}$. Then a $L R F$ $\rho$ has to satisfy (14) for any point of the form $\mathbf{x}^{\prime \prime}+a \mathbf{y}^{\prime \prime}$ with integer $a>0$ since it is a point in $I(\mathcal{Q})$; letting $a$ grow to infinity, we see that (14) implies the homogeneous inequality (16). Similarly, (15) implies (17).

Definition 3.4. Given $X \subseteq I(\mathcal{Q})$ and $Y \subseteq I\left(\mathcal{R}_{\mathcal{Q}}\right)$, define

$$
W S(X, Y)=\bigcup_{\mathbf{x}^{\prime \prime} \in X} W\left(\mathbf{x}^{\prime \prime}\right) \cup \bigcup_{\mathbf{y}^{\prime \prime} \in Y} W_{H}\left(\mathbf{y}^{\prime \prime}\right) .
$$


LEMMA 3.5. Let $X \subseteq I(\mathcal{Q}), X \neq \emptyset$, and $Y \subseteq I\left(\mathcal{R}_{\mathcal{Q}}\right)$. If $W S(X, Y)=\mathbb{Q}^{n+1}$, then there is no LRF for $I(\mathcal{Q})$.

Proof. Let $W S(X, Y)=\mathbb{Q}^{n+1}$. For any $\left(\lambda_{0}, \vec{\lambda}\right) \in \mathbb{Q}^{n+1}$, we prove that $\rho(\mathbf{x})=\vec{\lambda} \cdot \mathbf{x}+\lambda_{0}$ is not a $L R F$. If $\left(\lambda_{0}, \vec{\lambda}\right) \in W\left(\mathbf{x}^{\prime \prime}\right)$ for some $\mathbf{x}^{\prime \prime} \in X$, then the conclusion is clear since conditions 1415 do not hold. Otherwise, suppose that $\left(\lambda_{0}, \vec{\lambda}\right) \in W_{H}\left(\mathbf{y}^{\prime \prime}\right)$ for $\mathbf{y}^{\prime \prime} \in Y$. Thus, $\mathbf{y}^{\prime \prime}$ fails to satisfy one of conditions (16 17). Next we show that, in such case, there must exist $\mathbf{z}^{\prime \prime} \in I(\mathcal{Q})$ that fails either (14) or (15). In this part of the proof, we rely on the fact that $X \neq \emptyset$.

Case 1: Suppose (16) is not satisfied. That is, $\vec{\lambda} \cdot \mathbf{y}<0$.

Choose $\mathbf{x}^{\prime \prime} \in X$, and note that $\rho(\mathbf{x}) \geq 0$, otherwise $\left(\lambda_{0}, \vec{\lambda}\right) \in W\left(\mathbf{x}^{\prime \prime}\right)$ which we have assumed not true. Note that for any integer $a \geq 0$, the integer point $\mathbf{z}^{\prime \prime}=\mathbf{x}^{\prime \prime}+a \cdot \mathbf{y}^{\prime \prime}$ is a transition in $I(\mathcal{Q})$, and $\mathbf{z}^{\prime \prime}=\left(\begin{array}{c}\mathbf{x}+a \cdot \mathbf{y} \\ \mathbf{x}^{\prime}+a \cdot \mathbf{y}^{\prime}\end{array}\right)$. We choose $a$ as an integer sufficiently large so that $a \cdot(\vec{\lambda} \cdot \mathbf{y}) \leq-(1+\rho(\mathbf{x}))$. Now,

$$
\begin{aligned}
\rho(\mathbf{z}) & =\vec{\lambda} \cdot(\mathbf{x}+a \cdot \mathbf{y})+\lambda_{0} \\
& =\rho(\mathbf{x})+a \cdot(\vec{\lambda} \cdot \mathbf{y}) \leq \rho(\mathbf{x})-(1+\rho(\mathbf{x}))=-1
\end{aligned}
$$

So $\rho$ fails $(14)$ on $\mathbf{z}^{\prime \prime} \in I(\mathcal{Q})$, and thus cannot be a $L R F$.

Case 2: Suppose (17) is not satisfied. That is, $\vec{\lambda} \cdot\left(\mathbf{y}-\mathbf{y}^{\prime}\right)<0$.

Choose $\mathbf{x}^{\prime \prime} \in X$, and note that $\rho(\mathbf{x})-\rho\left(\mathbf{x}^{\prime}\right) \geq 1$, otherwise $\left(\lambda_{0}, \vec{\lambda}\right) \in W\left(\mathbf{x}^{\prime \prime}\right)$ which we have assumed not true. Define $\mathbf{z}^{\prime \prime}$ as above, but now choosing $a$ sufficiently large to make $a \cdot\left(\vec{\lambda} \cdot\left(\mathbf{y}-\mathbf{y}^{\prime}\right)\right) \leq$ $-\left(1+\rho(\mathbf{x})-\rho\left(\mathbf{x}^{\prime \prime}\right)\right)$. Now,

$$
\begin{aligned}
\rho(\mathbf{z})-\rho\left(\mathbf{z}^{\prime}\right) & =\vec{\lambda} \cdot\left((\mathbf{x}+a \cdot \mathbf{y})-\left(\mathbf{x}^{\prime}+a \cdot \mathbf{y}^{\prime}\right)\right) \\
& =\rho(\mathbf{x})-\rho\left(\mathbf{x}^{\prime}\right)+a \cdot\left(\vec{\lambda} \cdot\left(\mathbf{y}-\mathbf{y}^{\prime}\right)\right) \\
& \leq \rho(\mathbf{x})-\rho\left(\mathbf{x}^{\prime}\right)-\left(1+\rho(\mathbf{x})-\rho\left(\mathbf{x}^{\prime}\right)\right)=-1
\end{aligned}
$$

So $\rho$ fails 15 on $\mathbf{z}^{\prime \prime} \in I(\mathcal{Q})$, and thus cannot be a $L R F$.

Note that the condition $W S(X, Y)=\mathbb{Q}^{n+1}$ is equivalent to saying that the conjunction of inequalities 14 15), for all $\mathbf{x}^{\prime \prime} \in X$, and inequalities 16[17), for all $\mathbf{y}^{\prime \prime} \in Y$, has no (rational) solution. We denote this set of inequalities by $\Psi_{W S}(X, Y)$. Note that the variables in $\Psi_{W S}(X, Y)$ are $\lambda_{0}, \ldots, \lambda_{n}$, which range over $\mathbb{Q}$, and thus, the test that it has no solution can be done in polynomial time since it is an $L P$ problem over the rationals.

EXAMPLE 3.6. Consider the following integer $S L C$ loop:

$$
\text { while }\left(x_{1} \geq 0\right) \text { do } x_{1}^{\prime}=x_{1}+x_{2}, x_{2}^{\prime}=x_{2}-1
$$

Let $\mathbf{x}_{1}^{\prime \prime}=(0,2,2,1)^{\mathrm{T}} \in I(\mathcal{Q})$ and $\mathbf{y}_{1}^{\prime \prime}=(1,-2,-1,-2)^{\mathrm{T}} \in I\left(\mathcal{R}_{\mathcal{Q}}\right)$. Then, $\Psi_{W S}\left(\left\{\mathbf{x}_{1}^{\prime \prime}\right\},\left\{\mathbf{y}_{1}^{\prime \prime}\right\}\right)$ is a conjunction of the inequalities

$$
\left\{2 \lambda_{2}+\lambda_{0} \geq 0,-2 \lambda_{1}+\lambda_{2} \geq 1, \lambda_{1}-2 \lambda_{2} \geq 0,2 \lambda_{1} \geq 0\right\}
$$

The first two inequalities correspond to applying (14 15) to $\mathbf{x}_{1}^{\prime \prime}$, and the other ones to applying (16 17) to $\mathbf{y}_{1}^{\prime \prime}$. It is easy to verify that $19 p$ is not satisfiable, thus, $W S\left(\left\{\mathbf{x}_{1}^{\prime \prime}\right\},\left\{\mathbf{y}_{1}^{\prime \prime}\right\}\right)=\mathbb{Q}^{3}$ and the loop does not have a $L R F$. This is a classical loop for which there is no $L R F$. 
Lemma 3.5 provides a sufficient condition for the nonexistence of $L R F$, the next lemma shows that this condition is also necessary. In particular, it shows that if there is no $L R F$ for $I(\mathcal{Q})$, then the vertices and rays of $\mathcal{Q}_{I}$ serve as $X$ and $Y$ of Lemma 3.5 respectively.

LEMMA 3.7. Let the integer hull of the transition polyhedron $\mathcal{Q}$ be $\mathcal{Q}_{I}=\operatorname{convhull}\left\{\mathbf{x}_{1}^{\prime \prime}, \ldots, \mathbf{x}_{m}^{\prime \prime}\right\}+$ cone $\left\{\mathbf{y}_{1}^{\prime \prime}, \ldots, \mathbf{y}_{t}^{\prime \prime}\right\}$. If there is no LRF for $I(\mathcal{Q})$, then $W S\left(\left\{\mathbf{x}_{1}^{\prime \prime}, \ldots, \mathbf{x}_{m}^{\prime \prime}\right\},\left\{\mathbf{y}_{1}^{\prime \prime}, \ldots, \mathbf{y}_{t}^{\prime \prime}\right\}\right)=\mathbb{Q}^{n+1}$.

Proof. We prove the contra-positive. Suppose that

$$
W S\left(\left\{\mathbf{x}_{1}^{\prime \prime}, \ldots, \mathbf{x}_{m}^{\prime \prime}\right\},\left\{\mathbf{y}_{1}^{\prime \prime}, \ldots, \mathbf{y}_{t}^{\prime \prime}\right\}\right) \neq \mathbb{Q}^{n+1}
$$

Then, there is $\left(\lambda_{0}, \vec{\lambda}\right) \in \mathbb{Q}^{n+1}$ that fulfills 1415 for all $\mathbf{x}_{i}^{\prime \prime}$ and $\sqrt{16} 17$ for all $\mathbf{y}_{j}^{\prime \prime}$. We claim that $\rho(\mathbf{x})=\vec{\lambda} \cdot \mathbf{x}+\lambda_{0}$ is a $L R F$ for $I(\mathcal{Q})$.

To see this, let $\mathbf{x}^{\prime \prime}$ be an arbitrary point of $I(\mathcal{Q})$. Then $\mathbf{x}^{\prime \prime}=\sum_{i=1}^{m} a_{i} \cdot \mathbf{x}_{i}^{\prime \prime}+\sum_{j=1}^{t} b_{j} \cdot \mathbf{y}_{j}^{\prime \prime}$ for some $a_{i}, b_{j} \geq 0$ where $\sum_{i=1}^{m} a_{i}=1$. Now, we show that $\mathbf{x}^{\prime \prime}$ and $\rho$ satisfy 1415 which means that $\rho$ is a $L R F$ for $I(\mathcal{Q})$ :

$$
\begin{aligned}
\vec{\lambda} \cdot \mathbf{x}+\lambda_{0} & =\lambda_{0}+\sum_{i=1}^{m} a_{i} \cdot\left(\vec{\lambda} \cdot \mathbf{x}_{i}\right)+\sum_{j=1}^{t} b_{j} \cdot\left(\vec{\lambda} \cdot \mathbf{y}_{j}\right) \\
& =\sum_{i=1}^{m} a_{i} \cdot\left(\vec{\lambda} \cdot \mathbf{x}_{i}+\lambda_{0}\right)+\sum_{j=1}^{t} b_{j} \cdot\left(\vec{\lambda} \cdot \mathbf{y}_{j}\right) \\
& \geq 0+0=0 \\
\vec{\lambda} \cdot\left(\mathbf{x}-\mathbf{x}^{\prime}\right) & =\sum_{i=1}^{m} a_{i} \cdot\left(\vec{\lambda} \cdot\left(\mathbf{x}_{i}-\mathbf{x}_{i}^{\prime}\right)\right)+\sum_{j=1}^{t} b_{j} \cdot\left(\vec{\lambda} \cdot\left(\mathbf{y}_{j}-\mathbf{y}_{j}^{\prime}\right)\right) \\
& \geq 1+0=1
\end{aligned}
$$

Note that the solutions of $\Psi_{W S}\left(\left\{\mathbf{x}_{1}^{\prime \prime}, \ldots, \mathbf{x}_{m}^{\prime \prime}\right\},\left\{\mathbf{y}_{1}^{\prime \prime}, \ldots, \mathbf{y}_{t}^{\prime \prime}\right\}\right)$ in Lemma 3.7 actually define the set of all LRFs for $I(\mathcal{Q})$. We will address this point later in Section 3.4 for synthesizing LRFs.

EXAMPLE 3.8. Consider again the loop of Example 3.6, and recall that it does not have a $L R F$. The generator representation of $\mathcal{Q}_{I}$ is

$$
\mathcal{Q}_{I}=\operatorname{convhull}\left\{\mathbf{x}_{1}^{\prime \prime}\right\}+\operatorname{cone}\left\{\mathbf{y}_{1}^{\prime \prime}, \mathbf{y}_{2}^{\prime \prime}, \mathbf{y}_{3}^{\prime \prime}\right\}
$$

where $\mathbf{x}_{1}^{\prime \prime}=(0,1,1,0)^{\mathrm{T}}, \mathbf{y}_{1}^{\prime \prime}=(0,-1,-1,-1)^{\mathrm{T}}, \mathbf{y}_{2}^{\prime \prime}=(0,1,1,1)^{\mathrm{T}}$ and $\mathbf{y}_{3}^{\prime \prime}=(1,-1,0,-1)^{\mathrm{T}}$. Then, $\Psi_{W S}\left(\left\{\mathbf{x}_{1}^{\prime \prime}\right\},\left\{\mathbf{y}_{1}^{\prime \prime}, \mathbf{y}_{2}^{\prime \prime}, \mathbf{y}_{3}^{\prime \prime}\right\}\right)$ is a conjunction of the following inequalities

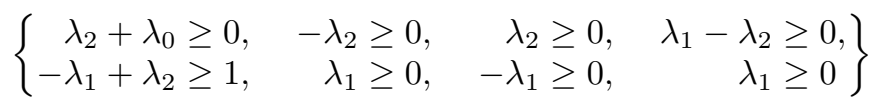

The inequalities in the leftmost column correspond to applying $\left(14[15)\right.$ to $\mathbf{x}_{1}^{\prime \prime}$, and those in the other columns to applying (16 17) to $\mathbf{y}_{1}^{\prime \prime}, \mathbf{y}_{2}^{\prime \prime}$, and $\mathbf{y}_{3}^{\prime \prime}$ respectively. It is easy to verify that 20 is not satisfiable, and thus, $W S\left(\left\{\mathbf{x}_{1}^{\prime \prime}\right\},\left\{\mathbf{y}_{1}^{\prime \prime}, \mathbf{y}_{2}^{\prime \prime}, \mathbf{y}_{3}^{\prime \prime}\right\}\right)=\mathbb{Q}^{3}$. 
Lemmas 3.5 and 3.7 provide a necessary and sufficient condition for the nonexistence of a $L R F$.

Corollary 3.9. There is no $L R F$ for $I(\mathcal{Q})$ if and only if there are two finite sets $X \subseteq I(\mathcal{Q}), X \neq \emptyset$, and $Y \subseteq I\left(\mathcal{R}_{\mathcal{P}}\right)$, such that $W S(X, Y)=\mathbb{Q}^{n+1}$.

The next lemma concerns the bit-size of the witness.

LEMMA 3.10. If there exists a witness for the nonexistence of a $L R F$ for $I(\mathcal{Q})$, there exists one with $X \subseteq I(\mathcal{Q})$ and $Y \subseteq I\left(\mathcal{R}_{\mathcal{Q}}\right)$ such that $|X|+|Y| \leq n+2$; and its bit-size is polynomially bounded in the bit-size of the input.

Proof. Recall that by Lemma 3.7, if $I(\mathcal{Q})$ has no $L R F$, then

$$
W S\left(\left\{\mathbf{x}_{1}^{\prime \prime}, \ldots, \mathbf{x}_{m}^{\prime \prime}\right\},\left\{\mathbf{y}_{1}^{\prime \prime}, \ldots, \mathbf{y}_{t}^{\prime \prime}\right\}\right)=\mathbb{Q}^{n+1}
$$

or, equivalently, $\Psi_{W S}\left(\left\{\mathbf{x}_{1}^{\prime \prime}, \ldots, \mathbf{x}_{m}^{\prime \prime}\right\},\left\{\mathbf{y}_{1}^{\prime \prime}, \ldots, \mathbf{y}_{t}^{\prime \prime}\right\}\right)$ has no solution. A corollary of Farkas' Lemma Schrijver 1986, p. 94] states that if a finite set of inequalities over $\mathbb{Q}^{d}$, for some $d>0$, has no solution, there is a subset of at most $d+1$ inequalities that has no solution. Since the set of inequalities $\Psi_{W S}\left(\left\{\mathbf{x}_{1}^{\prime \prime}, \ldots, \mathbf{x}_{m}^{\prime \prime}\right\},\left\{\mathbf{y}_{1}^{\prime \prime}, \ldots, \mathbf{y}_{t}^{\prime \prime}\right\}\right)$ is over $\mathbb{Q}^{n+1}$, there is a subset of at most $n+2$ inequalities that has no solution. This subset involves at most $n+2$ integer points out of $\left\{\mathbf{x}_{1}^{\prime \prime}, \ldots, \mathbf{x}_{m}^{\prime \prime}\right\}$ and $\left\{\mathbf{y}_{1}^{\prime \prime}, \ldots, \mathbf{y}_{t}^{\prime \prime}\right\}$, because every inequality in $\Psi_{W S}\left(\left\{\mathbf{x}_{1}^{\prime \prime}, \ldots, \mathbf{x}_{m}^{\prime \prime}\right\},\left\{\mathbf{y}_{1}^{\prime \prime}, \ldots, \mathbf{y}_{t}^{\prime \prime}\right\}\right)$ is defined by either one $\mathbf{x}_{i}^{\prime \prime}$ or $\mathbf{y}_{i}^{\prime \prime}$ (see 114 17)). Let these points be $X$ and $Y$, then $|X|+|Y| \leq n+2$ and $\Psi_{W S}(X, Y)$ has no solution, i.e., $W S(X, Y)=\mathbb{Q}^{n+1}$. Moreover it must be that case that $X \neq \emptyset$, since all constraints of the type 16 17] are satisfied by $\left(\lambda_{0}, \vec{\lambda}\right)=\mathbf{0}^{\mathrm{T}}$.

Now we show that $X$ and $Y$ may be chosen to have bit-size polynomial in the size of the input. Recall that the input is the set of inequalities $A^{\prime \prime} \mathbf{x}^{\prime \prime} \leq \mathbf{c}^{\prime \prime}$ that define $\mathcal{Q}$, and its bit-size is $\|\mathcal{Q}\|_{b}$. Recall that the points of $X$ and $Y$ in Lemma 3.7 come from the generator representation, and that there is a generator representation in which each vertex/ray can fit in $\left\|\mathcal{Q}_{I}\right\|_{v}$ bits. Thus, the bit-size of $X$ and $Y$ may be bounded by $(n+2) \cdot\left\|\mathcal{Q}_{I}\right\|_{v}$. By Theorem 2.2 , since the dimension of $\mathcal{Q}$ is $2 n$,

$$
(n+2) \cdot\left\|\mathcal{Q}_{I}\right\|_{v} \leq(n+2) \cdot\left(6 \cdot(2 n)^{3} \cdot\|\mathcal{Q}\|_{f}\right) \leq\left(48 n^{4}+96 n^{3}\right) \cdot\|\mathcal{Q}\|_{b}
$$

which is polynomial in the bit-size of the input.

EXAMPLE 3.11. Consider $\Psi_{W S}\left(\left\{\mathbf{x}_{1}^{\prime \prime}\right\},\left\{\mathbf{y}_{1}^{\prime \prime}, \mathbf{y}_{2}^{\prime \prime}, \mathbf{y}_{3}^{\prime \prime}\right\}\right)$ of Example 3.8 It is easy to see that the inequalities $-\lambda_{2} \geq 0, \lambda_{1} \geq 0$ and $-\lambda_{1}+\lambda_{2} \geq 1$ are enough for unsatisfiability $(n+1$ inequalities, since $n=2$ ). These inequalities correspond to $\mathbf{x}_{1}^{\prime \prime}$ and $\mathbf{y}_{1}^{\prime \prime}$, and thus, these two points witness the nonexistence of a $L R F$ (note that this witness consists, in this example, of less than $n+2$ points).

THEOREM 3.12. $\operatorname{LinRF}(\mathbb{Z}) \in \operatorname{coNP}$ for $S L C$ loops.

Proof. We show that the complement of $\operatorname{LinRF}(\mathbb{Z})$ has a polynomially checkable witness. The witness is a listing of sets $X$ and $Y$ of at most $n+2$ elements and has a polynomial bit-size (specifically, a bit-size bounded as in Lemma 3.10p. Verifying a witness consists of the following steps:

Step 1. Verify that each $\mathbf{x}^{\prime \prime} \in X$ is in $I(\mathcal{Q})$, which can be done by verifying $A^{\prime \prime} \mathbf{x}^{\prime \prime} \leq \mathbf{c}^{\prime \prime}$; and that each $\mathbf{y}^{\prime \prime} \in Y$ is in $I\left(\mathcal{R}_{\mathcal{Q}}\right)$, which can be done by verifying $A^{\prime \prime} \mathbf{y}^{\prime \prime} \leq \mathbf{0}$. This is done in polynomial time. Note that according to Lemma 3.5 it is not necessary to check that $X$ and $Y$ come from a particular generator representation. 
Step 2. Verify that $W S(X, Y)=\mathbb{Q}^{n+1}$. This can be done by checking that $\Psi_{W S}(X, Y)$ has no solutions, which can be done in polynomial time since it is an $L P$ problem over $\mathbb{Q}^{n+1}$.

\subsection{Inclusion in coNP for $M L C$ loops}

In this section we consider the inclusion in coNP for $M L C$ loops. For this, we assume an input $M L C$ loop with transition polyhedra $\mathcal{Q}_{1}, \ldots, \mathcal{Q}_{k}$ where each $\mathcal{Q}_{i}$ is specified by $A_{i}^{\prime \prime} \mathbf{x}^{\prime \prime} \leq \mathbf{c}_{i}^{\prime \prime}$.

The proof follows the structure of the $S L C$ case. The main difference is that points of the witness may come from different transition polyhedra. Namely, $X=X_{1} \cup \cdots \cup X_{k}$ and $Y=Y_{1} \cup \cdots \cup Y_{k}$ where each $X_{i} \subseteq I\left(\mathcal{Q}_{i}\right)$ and $Y_{i} \subseteq I\left(\mathcal{R}_{\mathcal{Q}_{i}}\right)$. Lemmas 3.5, 3.7, and 3.10, Corollary 3.9, and Theorem 3.12, are rewritten in terms of such witnesses as follows (the proofs are the same unless stated otherwise).

LEMMA 3.13. Let $X=X_{1} \cup \cdots \cup X_{k}$ and $Y=Y_{1} \cup \cdots \cup Y_{k}$, where $X_{i} \subseteq I\left(\mathcal{Q}_{i}\right), Y_{i} \subseteq I\left(\mathcal{R}_{\mathcal{Q}_{i}}\right)$ and $Y_{i} \neq \emptyset \Rightarrow X_{i} \neq \emptyset$. If $W S(X, Y)=\mathbb{Q}^{n+1}$, then there is no $\operatorname{LRF}$ for $I\left(\mathcal{Q}_{1}\right), \ldots, I\left(\mathcal{Q}_{k}\right)$.

Note that $W S(X, Y)=\bigcup_{i=1}^{k} W S\left(X_{i}, Y_{i}\right)$ and that in the proof of Lemma 3.5 (when re-used to obtain the above lemma) it is necessary to use the condition $Y_{i} \neq \emptyset \Rightarrow X_{i} \neq \emptyset$.

LEMMA 3.14. For $1 \leq i \leq k$, let $\mathcal{Q}_{i I}=\operatorname{convhull}\left\{X_{i}\right\}+\operatorname{cone}\left\{Y_{i}\right\}$ be the integer hull of $\mathcal{Q}_{i}$, and define $X=X_{1} \cup \cdots \cup X_{k}$ and $Y=Y_{1} \cup \cdots \cup Y_{k}$. If there is no LRF for $I\left(\mathcal{Q}_{1}\right), \ldots, I\left(\mathcal{Q}_{k}\right)$, then $W S(X, Y)=\mathbb{Q}^{n+1}$.

Proof. The proof follows that of Lemma 3.7. We pick $\left(\lambda_{0}, \vec{\lambda}\right) \in \mathbb{Q}^{n+1} \backslash W S(X, Y)$ and show that $\rho(\mathbf{x})=\vec{\lambda} \cdot \mathbf{x}+\lambda_{0}$ is a $L R F$ for all $I\left(\mathcal{Q}_{i}\right)$. This is accomplished by performing the same calculation, however referring to $X_{i}$ and $Y_{i}$ when proving that $\rho$ is a $L R F$ for $I\left(\mathcal{Q}_{i}\right)$.

Corollary 3.15. There is no $L R F$ for $I\left(\mathcal{Q}_{1}\right), \ldots, I\left(\mathcal{Q}_{k}\right)$, if and only if there are two finite sets $X=$ $X_{1} \cup \cdots \cup X_{k}$ and $Y=Y_{1} \cup \cdots \cup Y_{k}$, where $X_{i} \subseteq I\left(\mathcal{Q}_{i}\right)$ and $Y_{i} \subseteq I\left(\mathcal{R}_{\mathcal{Q}_{i}}\right)$, and $Y_{i} \neq \emptyset \Rightarrow X_{i} \neq \emptyset$, such that $W S(X, Y)=\mathbb{Q}^{n+1}$.

LEMMA 3.16. If there exists a witness for the nonexistence of a LRF for $I\left(\mathcal{Q}_{1}\right), \ldots, I\left(\mathcal{Q}_{k}\right)$, then there exists one with $X=X_{1} \cup \cdots \cup X_{k}$ and $Y=Y_{1} \cup \cdots \cup Y_{k}$, where $X_{i} \subseteq I\left(\mathcal{Q}_{i}\right)$ and $Y_{i} \subseteq I\left(\mathcal{R}_{\mathcal{Q}_{i}}\right)$, such that $\sum_{i=1}^{k}\left(\left|X_{i}\right|+\left|Y_{i}\right|\right) \leq 2 n+3$; and its bit-size is polynomially bounded in the bit-size of the input.

Proof. Let $\hat{X}_{i}, \hat{Y}_{i}$ be the generators of $\mathcal{Q}_{i I}$. First, as in Lemma 3.10 , we argue that there is a set of at most $n+2$ inequalities out of $\Psi_{W S}\left(\bigcup \hat{X}_{i}, \bigcup \hat{Y}_{i}\right)$ that have no solution. These inequalities correspond to $n+2$ points out of the sets $\hat{X}_{i}, \hat{Y}_{i}$. Let $X_{i}$ (respectively $Y_{i}$ ) be the set of points that come from $\hat{X}_{i}$ (respectively $\left.\hat{Y}_{i}\right)$. Since $\left(\lambda_{0}, \vec{\lambda}\right)=\mathbf{0}^{\mathrm{T}}$ is not a solution, at least one of the points must come from a set $\hat{X}_{i}$. But $n+1$ other points might come from sets $\hat{Y}_{i}$. Since a witness must satisfy $Y_{i} \neq \emptyset \Rightarrow X_{i} \neq \emptyset$, we may have to add $n+1$ points to form a valid witness, for a total of $2 n+3$ (clearly, $n+1$ can be replaced by $k$ when $k<n+1)$. Bounding the bit-size of the witness is done as in Lemma 3.10, but using the $2 n+3$ instead of $n+2$, and $\max _{i}\left\|\mathcal{Q}_{i}\right\|_{b}$ instead of $\|\mathcal{Q}\|_{b}$.

THEOREM 3.17. $\operatorname{LinRF}(\mathbb{Z}) \in \operatorname{coNP}$.

Proof. Almost identical to the proof of Theorem 3.12. Note that the witness is given as $X=X_{1} \cup$ $\cdots \cup X_{k}$ and $Y=Y_{1} \cup \cdots \cup Y_{k}$, and the verifier should use the appropriate set of constraints to check that each $\mathbf{x}^{\prime \prime} \in X_{i}$ is in $I\left(\mathcal{Q}_{i}\right)$, and that each $\mathbf{y}^{\prime \prime} \in Y_{i}$ is in $I\left(\mathcal{R}_{\mathcal{Q}_{i}}\right)$. 
EXAMPLE 3.18. Consider again the integer $M L C$ loop (3) from Section 1. It is a classical $M L C$ loop for which there is no $L R F$. The integer hulls of the corresponding transition polyhedra are

$$
\begin{aligned}
& \mathcal{Q}_{1 I}=\operatorname{convhull}\left\{\mathbf{x}_{1}^{\prime \prime}\right\}+\operatorname{cone}\left\{\mathbf{y}_{1}^{\prime \prime}, \mathbf{y}_{2}^{\prime \prime}, \mathbf{y}_{3}^{\prime \prime}, \mathbf{y}_{4}^{\prime \prime}\right\} \\
& \mathcal{Q}_{2 I}=\operatorname{convhull}\left\{\mathbf{x}_{2}^{\prime \prime}\right\}+\operatorname{cone}\left\{\mathbf{y}_{5}^{\prime \prime}, \mathbf{y}_{6}^{\prime \prime}\right\}
\end{aligned}
$$

where

$$
\begin{array}{llll}
\mathbf{x}_{1}^{\prime \prime}=(0,0,-1,0)^{\mathrm{T}} & \mathbf{y}_{1}^{\prime \prime}=(0,0,0,-1)^{\mathrm{T}} & \mathbf{y}_{3}^{\prime \prime}=(0,1,0,0)^{\mathrm{T}} & \mathbf{y}_{5}^{\prime \prime}=(0,1,0,1)^{\mathrm{T}} \\
\mathbf{x}_{2}^{\prime \prime}=(0,0,0,-1)^{\mathrm{T}} & \mathbf{y}_{2}^{\prime \prime}=(0,0,0,1)^{\mathrm{T}} & \mathbf{y}_{4}^{\prime \prime}=(1,0,1,0)^{\mathrm{T}} & \mathbf{y}_{6}^{\prime \prime}=(1,0,1,0)^{\mathrm{T}}
\end{array}
$$

Let us first consider each path separately. We get

$$
\begin{aligned}
& \Psi_{W S}\left(\left\{\mathbf{x}_{1}^{\prime \prime}\right\},\left\{\mathbf{y}_{1}^{\prime \prime}, \mathbf{y}_{2}^{\prime \prime}, \mathbf{y}_{3}^{\prime \prime}\right\}\right)=\left\{\lambda_{0} \geq 0, \lambda_{1} \geq 1, \lambda_{2} \geq 0,-\lambda_{2} \geq 0\right\} \\
& \Psi_{W S}\left(\left\{\mathbf{x}_{2}^{\prime \prime}\right\},\left\{\mathbf{y}_{4}^{\prime \prime}, \mathbf{y}_{5}^{\prime \prime}, \mathbf{y}_{6}^{\prime \prime}\right\}\right)=\left\{\lambda_{0} \geq 0, \lambda_{2} \geq 1\right\}
\end{aligned}
$$

Both (21) and (22) are satisfiable. In fact, their solutions define the corresponding LRFs for each path when considered separately. For the $M L C$ loop, we have that $\Psi_{W S}\left(\left\{\mathbf{x}_{1}^{\prime \prime}, \mathbf{x}_{2}^{\prime \prime}\right\},\left\{\mathbf{y}_{1}^{\prime \prime}, \ldots, \mathbf{y}_{6}^{\prime \prime}\right\}\right)$ is the conjunction of the inequalities in (21) and (22), which is not satisfiable. Thus, while each path has a $L R F$, the $M L C$ loop does not. Note that the inequalities $\lambda_{2} \geq 1$ and $-\lambda_{2} \geq 0$ are enough to get unsatisfiability of $(2122)$, thus, a possible witness is $X_{1}=\left\{\mathbf{x}_{1}^{\prime \prime}\right\}, Y_{1}=\left\{\mathbf{y}_{2}^{\prime \prime}\right\}, X_{2}=\left\{\mathbf{x}_{2}^{\prime \prime}\right\}, Y_{2}=\emptyset$. Note that it consists of less than $2 n+3$ points (as $n=2$ ).

\subsection{Synthesizing a linear ranking function}

Although the existence of a $L R F$ suffices for proving termination, generating a complete representation of the $L R F$ is important in some contexts, for instance complexity analysis where a ranking function provides an upper bound on the number of iterations that a loop can perform. In this section we give a complete algorithm that generates $L R F s$ for $M L C$ loops given by transition polyhedra $\mathcal{Q}_{1}, \ldots, \mathcal{Q}_{k}$. The following lemma is directly implied by lemmas 3.13 and 3.14 .

LEMMA 3.19. For $1 \leq i \leq k$, let $\mathcal{Q}_{i I}=\operatorname{convhull}\left\{X_{i}\right\}+\operatorname{cone}\left\{Y_{i}\right\}$ be the integer hull of $\mathcal{Q}_{i}$, and define $X=X_{1} \cup \cdots \cup X_{k}$ and $Y=Y_{1} \cup \cdots \cup Y_{k}$. Then, $\rho(\mathbf{x})=\vec{\lambda} \cdot \mathbf{x}+\lambda_{0}$ is a LRF for $I\left(\mathcal{Q}_{1}\right), \ldots, I\left(\mathcal{Q}_{k}\right)$, if and only if $\left(\lambda_{0}, \vec{\lambda}\right)$ is a solution of $\Psi_{W S}(X, Y)$.

The following algorithm follows: (1) Compute the generator representation for each $\mathcal{Q}_{i I}$; (2) Construct $\Psi_{W S}(X, Y)$; and (3) Use $L P$ to find a solution $\left(\lambda_{0}, \vec{\lambda}\right)$ for $\Psi_{W S}(X, Y)$.

EXAMPLE 3.20. Consider again Loop (1) from Section 1. The integer hull of the transition polyhedron is

$$
\mathcal{Q}_{I}=\operatorname{convhull}\left\{\mathbf{x}_{1}^{\prime \prime}, \mathbf{x}_{2}^{\prime \prime}\right\}+\operatorname{cone}\left\{\mathbf{y}_{1}^{\prime \prime}, \mathbf{y}_{2}^{\prime \prime}\right\}
$$

where $\mathbf{x}_{1}^{\prime \prime}=(1,1,1,0)^{\mathrm{T}}, \mathbf{x}_{2}^{\prime \prime}=(1,0,1,-1)^{\mathrm{T}}, \mathbf{y}_{1}^{\prime \prime}=(1,1,1,-1)^{\mathrm{T}}$, and $\mathbf{y}_{2}^{\prime \prime}=(1,-1,1,-3)^{\mathrm{T}}$. The formula $\Psi_{W S}\left(\left\{\mathbf{x}_{1}^{\prime \prime}, \mathbf{x}_{2}^{\prime \prime}\right\},\left\{\mathbf{y}_{1}^{\prime \prime}, \mathbf{y}_{2}^{\prime \prime}\right\}\right)$ is the conjunction of the following inequalities (we eliminated clearly redundant inequalities)

$$
\left\{\lambda_{1}+\lambda_{2}+\lambda_{0} \geq 0, \lambda_{1}+\lambda_{0} \geq 0, \lambda_{1}+\lambda_{2} \geq 0, \lambda_{1}-\lambda_{2} \geq 0, \lambda_{2} \geq 1\right\}
$$

which is satisfiable for $\lambda_{1}=\lambda_{2}=1$ and $\lambda_{0}=-1$, and therefore, $f\left(x_{1}, x_{2}\right)=x_{1}+x_{2}-1$ is a $L R F$. Note that the loop does not terminate when the variables range over $\mathbb{Q}$, e.g., for $x_{1}=x_{2}=\frac{1}{2}$ (see Figure 1(A)). 
EXAMPLE 3.21. Let us consider now Loop (2) from Section 1. The integer hull of the transition polyhedron is

$$
\mathcal{Q}_{I}=\operatorname{convhull}\left\{\mathbf{x}_{1}^{\prime \prime}, \mathbf{x}_{2}^{\prime \prime}, \mathbf{x}_{3}^{\prime \prime}, \mathbf{x}_{4}^{\prime \prime}, \mathbf{x}_{5}^{\prime \prime}, \mathbf{x}_{6}^{\prime \prime}\right\}+\operatorname{cone}\left\{\mathbf{y}_{1}^{\prime \prime}, \mathbf{y}_{2}^{\prime \prime}\right\}
$$

where

$$
\begin{array}{llll}
\mathbf{x}_{1}^{\prime \prime}=(4,16,1,16)^{\mathrm{T}} & \mathbf{x}_{3}^{\prime \prime}=(2,8,1,8)^{\mathrm{T}} & \mathbf{x}_{5}^{\prime \prime}=(4,1,1,1)^{\mathrm{T}} & \mathbf{y}_{1}^{\prime \prime}=(5,0,2,0)^{\mathrm{T}} \\
\mathbf{x}_{2}^{\prime \prime}=(1,4,0,4)^{\mathrm{T}} & \mathbf{x}_{4}^{\prime \prime}=(1,1,0,1)^{\mathrm{T}} & \mathbf{x}_{6}^{\prime \prime}=(2,1,1,1)^{\mathrm{T}} & \mathbf{y}_{2}^{\prime \prime}=(5,20,2,20)^{\mathrm{T}}
\end{array}
$$

The formula $\Psi_{W S}\left(\left\{\mathbf{x}_{1}^{\prime \prime}, \ldots, \mathbf{x}_{6}^{\prime \prime}\right\},\left\{\mathbf{y}_{1}^{\prime \prime}, \mathbf{y}_{2}^{\prime \prime}\right\}\right)$ is the conjunction of the following inequalities (we eliminated clearly redundant ones)

$$
\left\{\begin{array}{llll}
\lambda_{1} \geq 1, & 4 \lambda_{1}+\lambda_{2}+\lambda_{0} \geq 0, & 4 \lambda_{1}+16 \lambda_{2}+\lambda_{0} \geq 0, & 2 \lambda_{1}+\lambda_{2}+\lambda_{0} \geq 0, \\
5 \lambda_{1}+20 \lambda_{2} \geq 0, & 2 \lambda_{1}+8 \lambda_{2}+\lambda_{0} \geq 0, & \lambda_{1}+4 \lambda_{2}+\lambda_{0} \geq 0, & \lambda_{1}+\lambda_{2}+\lambda_{0} \geq 0
\end{array}\right\}
$$

which is satisfiable for $\lambda_{1}=1, \lambda_{2}=0$ and $\lambda_{0}=-1$, and therefore, $f\left(x_{1}, x_{2}\right)=x_{1}-1$ is a $L R F$. Note that this loop, too, does not terminate when the variables range over $\mathbb{Q}$, e.g., for $x_{1}=\frac{1}{4}$ and $x_{2}=1$ (see Figure 1(C)).

If we consider both loops (1) and (2) as two paths in an MLC loop, then to synthesize LRFs we use the conjunction of the inequalities in (23) and (24). In this case, $\lambda_{1}=\lambda_{2}=1$ and $\lambda_{0}=-1$, is a solution, but $\lambda_{1}=1, \lambda_{2}=0$ and $\lambda_{0}=-1$ is not. Therefore, $f\left(x_{1}, x_{2}\right)=x_{1}+x_{2}-1$ is a LRF for both paths, and thus for the $M L C$ loop, but not $f\left(x_{1}, x_{2}\right)=x_{1}-1$.

Given our hardness results, one cannot expect a polynomial-time algorithm. Indeed, constructing the generator representation of the integer hull of a polyhedron from the corresponding set of inequalities $A_{i}^{\prime \prime} \mathbf{x} \leq \mathbf{c}_{i}^{\prime \prime}$ may require exponential time - the number of generators itself may be exponential. Their bit-size, on the other hand, is polynomial by Theorem 2.2. This is interesting, since it yields:

Corollary 3.22. Consider an $M L C$ loop specified by the transition polyhedra $\mathcal{Q}_{1}, \ldots, \mathcal{Q}_{k}$, where each $\mathcal{Q}_{i}$ is specified by $A_{i}^{\prime \prime} \mathbf{x} \leq \mathbf{c}_{i}^{\prime \prime}$. If there is a $L R F$ for $I\left(\mathcal{Q}_{1}\right), \ldots, I\left(\mathcal{Q}_{k}\right)$, there is one whose bit-size is polynomial in the bit-size of $\left\{A_{i}^{\prime \prime} \mathbf{x} \leq \mathbf{c}_{i}^{\prime \prime}\right\}$, namely in $\max _{i}\left\|\mathcal{Q}_{i}\right\|_{b}$.

Proof. As in the last section, we bound the bit-size of each of the generators of $\mathcal{Q}_{i I}$ by $\left\|\mathcal{Q}_{i I}\right\|_{v} \leq$ $6(2 n)^{3} \cdot\left\|\mathcal{Q}_{i}\right\|_{f} \leq 48 n^{3} \cdot\left\|\mathcal{Q}_{i}\right\|_{b}$ for an appropriate $i$. This means that the bit-size of each equation in $\Psi_{W S}(X, Y)$, having one of the forms (14), (15), (16), or (17) is at most $5+48 n^{3} \cdot\left(\max _{i}\left\|\mathcal{Q}_{i}\right\|_{b}\right)$. Let $\mathcal{P}$ be the polyhedron defined by $\Psi_{W S}(X, Y)$, then $\|\mathcal{P}\|_{f} \leq 5+48 n^{3} \cdot\left(\max _{i}\left\|\mathcal{Q}_{i}\right\|_{b}\right)$. If $\Psi_{W S}(X, Y)$ has a solution, then any vertex of $\mathcal{P}$ is such a solution, and yields a $L R F$. Using Theorem 2.1, together with the above bound for $\|\mathcal{P}\|_{f}$ and the fact that the dimension of $\mathcal{P}$ is $n+1$, we conclude that there is a generator representation for $\mathcal{P}$ in which the bit-size $\|\mathcal{P}\|_{v}$ of the vertices is bounded as follows:

$$
\|\mathcal{P}\|_{v} \leq 4 \cdot(n+1)^{2} \cdot\|\mathcal{P}\|_{f} \leq 4 \cdot(n+1)^{2} \cdot\left(5+48 n^{3} \cdot\left(\max _{i}\left\|\mathcal{Q}_{i}\right\|_{b}\right)\right)
$$

This also bounds the bit-size of the corresponding $L R F$.

We conclude this section by noting that Lemma 3.19 works also for $\operatorname{LinRF}(\mathbb{Q})$, if we consider $\mathcal{Q}_{i}$ instead of $\mathcal{Q}_{i I}$. This can be easily proven by reworking the proofs of Lemmas 3.13 and 3.14 for the case of $\mathcal{Q}_{i}$ instead of $\mathcal{Q}_{i I}$. We did not develop this line since the main use of these definitions is proving the coNP-completeness for $\operatorname{LinRF}(\mathbb{Z})$. This, however, has an interesting consequence: $\operatorname{LinRF}(\mathbb{Q})$ is still PTIME even if the input loop is given in the generator representations form instead of the constraints form. Practically, implementations of polyhedra that use of the double description method, such as PPL Bagnara et al. 2008b, in which both the generators and constraint representations are kept at the same time, can use the algorithm of Lemma 3.19judiciously when it seems better than algorithms that use the constraints representation Mesnard and Serebrenik 2008; Podelski and Rybalchenko 2004. 


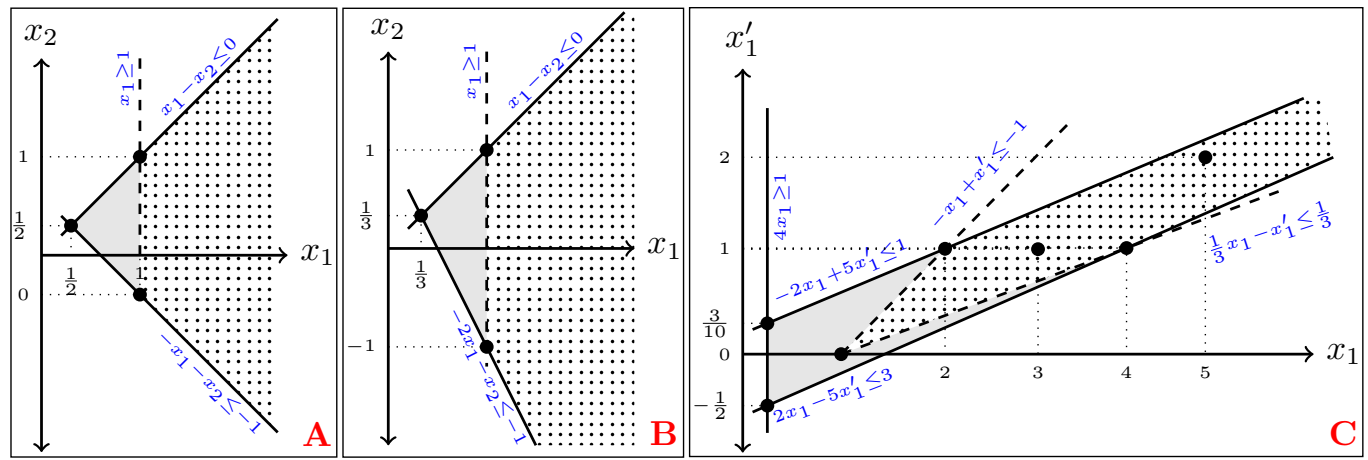

Figure 1: The polyhedra associated with three of our examples, projected to two dimensions. Dashed lines are added when computing the integer hull; dotted areas represent the integer hull; gray areas are rational points eliminated when computing the integer hull.

\section{Special Cases in PTIME}

In this section we discuss cases in which the $\operatorname{LinRF}(\mathbb{Z})$ problem is PTIME-decidable. We start by a basic observation: when the transition polyhedron of an $S L C$ loop is integral, the $\operatorname{LinRF}(\mathbb{Z})$ and $\operatorname{LinRF}(\mathbb{Q})$ problems are equivalent (a very similar statement stated by Cook et al. 2010, Lemma 3]).

LEMMA 4.1. Let $\mathcal{Q}$ be a transition polyhedron of a given $S L C$ loop, and let $\rho$ be a linear function. If $\mathcal{Q}$ is integral, then $\rho$ is a $L R F$ for $\mathcal{Q}$ if and only if $\rho$ is a $L R F$ for $I(\mathcal{Q})$.

Proof. Let $\mathcal{Q}$ be an integer polyhedron. $(\Rightarrow)$ Suppose that $\rho$ is a $L R F$ for $\mathcal{Q}$, then clearly it is also a $L R F$ for $I(\mathcal{Q})$ since $I(\mathcal{Q}) \subseteq \mathcal{Q}$. $(\Leftarrow)$ Suppose that $\rho$ is a $L R F$ for $I(\mathcal{Q})$, it thus satisfies $(7] 8)$ of Definition 2.3 for any integer point in $\mathcal{Q}$. However, by definition of an integer polyhedron, every rational point in $\mathcal{Q}$ is a convex combination of integer points from $I(\mathcal{Q})$, this proves that $\rho$ satisfies conditions $(78)$ for any rational point in $\mathcal{Q}$, as follows. Choose an arbitrary rational point $\mathbf{x}^{\prime \prime} \in \mathcal{Q}$. It can be written as $\mathbf{x}^{\prime \prime}=\sum a_{i} \cdot \mathbf{x}_{i}^{\prime \prime}$ where $a_{i}>0, \sum a_{i}=1$ and $\mathbf{x}_{i}^{\prime \prime} \in I(\mathcal{Q})$. Thus, $\mathbf{x}^{\prime \prime}=\left(\begin{array}{c}\sum a_{i} \cdot \mathbf{x}_{i} \\ \sum a_{i} \cdot \mathbf{x}_{i}^{\prime}\end{array}\right)$, and

$$
\begin{aligned}
\rho(\mathbf{x}) & =\left(\vec{\lambda} \cdot \sum a_{i} \cdot \mathbf{x}_{i}\right)+\lambda_{0}=\sum a_{i} \cdot\left(\vec{\lambda} \cdot \mathbf{x}_{i}+\lambda_{0}\right) \geq 0 \\
\Delta \rho\left(\mathbf{x}^{\prime \prime}\right) & =\left(\vec{\lambda} \cdot \sum a_{i} \cdot \mathbf{x}_{i}\right)-\left(\vec{\lambda} \cdot \sum a_{i} \cdot \mathbf{x}_{i}^{\prime}\right)=\sum a_{i} \cdot \vec{\lambda} \cdot\left(\mathbf{x}_{i}-\mathbf{x}_{i}^{\prime}\right) \geq 1
\end{aligned}
$$

The above lemma provides an alternative, and complete, procedure for $\operatorname{LinRF}(\mathbb{Z})$, namely, compute a constraint representation of its integer hull $Q_{I}$ and solve $\operatorname{LinRF}(\mathbb{Q})$. Note that computing the integer hull might require exponential time, and might also result in a polyhedron with an exponentially larger description. This means that the above procedure is exponential in general; but this concern is circumvented if the transition polyhedron is integral to begin with; and in special cases where it is known that computing the integer hull is easy. Formally, we call a class of polyhedra easy if computing its integer hull can be done in polynomial time. 
EXAMPLE 4.2. Consider again the $S L C$ loop (2) of Section 1 . The transition polyhedron is not integral, computing its integer hull adds the inequalities $-x_{1}+x_{1}^{\prime} \leq-1$ and $\frac{1}{3} x_{1}-x_{1}^{\prime} \leq \frac{1}{3}$. This is depicted in Figure $1(\mathrm{C})$. Applying $\operatorname{LinRF}(\mathbb{Q})$ on this loop does not find a $L R F$ since it does not terminate when the variables range over $\mathbb{Q}$, however, applying it on the integer hull finds the $L R F$ $f\left(x_{1}, x_{2}\right)=x_{1}-1$.

Corollary 4.3. The $\operatorname{LinRF}(\mathbb{Z})$ problem is PTIME-decidable for $S L C$ loops in which the transition polyhedron $\mathcal{Q}$ is guaranteed to be integral. This also applies to any easy class of polyhedra, namely a class where the integer hull is PTIME-computable.

Proof. Immediate from Lemma 4.1 and the fact that $\operatorname{LinRF}(\mathbb{Q})$ is PTIME-decidable.

Corollary 4.4. The $\operatorname{LinRF}(\mathbb{Z})$ problem is PTIME-decidable for $S L C$ loops in which the condition polyhedron $\mathcal{C}$ is guaranteed to be integral, or belongs to an easy class, and the update is linear with integer coefficients.

Proof. We show that if $\mathcal{C}$ is integral, the transition polyhedron $\mathcal{Q}$ is also integral, and thus Corollary 4.3 applies. Let the condition polyhedron $\mathcal{C}$ be integral, and the update be $\mathbf{x}^{\prime}=A^{\prime} \mathbf{x}+\mathbf{c}^{\prime}$ where the entries of $A^{\prime}$ and $\mathbf{c}^{\prime}$ are integer. Let $\mathbf{x}^{\prime \prime} \in \mathcal{Q}$, that is, $\mathbf{x} \in \mathcal{C}$ and $\mathbf{x}^{\prime}=A^{\prime} \mathbf{x}+\mathbf{c}^{\prime}$. Since $\mathcal{C}$ is integral, $\mathbf{x}$ is a convex combination of some integer points. I.e, $\mathbf{x}=\sum a_{i} \cdot \mathbf{x}_{i}$ where $a_{i}>0, \sum a_{i}=1$ and $\mathbf{x}_{i} \in I(\mathcal{C})$. Hence, $\mathbf{x}^{\prime}=A^{\prime}\left(\sum a_{i} \cdot \mathbf{x}_{i}\right)+\mathbf{c}^{\prime}=\sum a_{i} \cdot\left(A^{\prime} \mathbf{x}_{i}+\mathbf{c}^{\prime}\right)$ and

$$
\mathbf{x}^{\prime \prime}=\left(\begin{array}{l}
\mathbf{x} \\
\mathbf{x}^{\prime}
\end{array}\right)=\left(\begin{array}{c}
\sum a_{i} \cdot \mathbf{x}_{i} \\
\sum a_{i} \cdot\left(A^{\prime} \mathbf{x}_{i}+\mathbf{c}^{\prime}\right)
\end{array}\right)=\sum a_{i} \cdot\left(\begin{array}{c}
\mathbf{x}_{i} \\
A^{\prime} \mathbf{x}_{i}+\mathbf{c}^{\prime}
\end{array}\right)
$$

Now note that $\left(\begin{array}{c}\mathbf{x}_{i} \\ A^{\prime} \mathbf{x}_{i}+\mathbf{c}^{\prime}\end{array}\right)$ are integer points from $I(\mathcal{Q})$, which implies that $\mathbf{x}^{\prime \prime}$ is a convex combination of integer points in $\mathcal{Q}$. Hence, $\mathcal{Q}$ is integral.

Corollaries 4.3 and 4.4 suggest looking for classes of $S L C$ loops where we can easily ascertain that $\mathcal{Q}$ is integral, or that its integer hull can be computed in polynomial time. In what follows we address such cases: Section 4.1 discusses special cases in which the transition or condition polyhedron is integral by construction; Section 4.2 shows that for certain cases of two-variables per inequality constraints the integer hull can be computed in a polynomial time; Section 4.3 discusses the case of octagonal relations; Section 4.4 shows that for some cases $\operatorname{LinRF}(\mathbb{Z})$ is even strongly polynomial; and Section 4.5 extends the results to $M L C$ loops.

\subsection{Loops specified by integer polyhedra}

There are some well-known examples of polyhedra that are known to be integral due to some structural property. This gives us classes of $S L C$ loops where $\operatorname{LinRF}(\mathbb{Z})$ is in PTIME. The examples below follows Schrijver [1986], where the proofs of the lemmas can be found.

LEMMA 4.5. For any rational matrix $B$, the cone $\{\mathbf{x} \mid B \mathbf{x} \leq \mathbf{0}\}$ is an integer polyhedron.

Corollary 4.6. The $\operatorname{LinRF}(\mathbb{Z})$ problem is PTIME-decidable for $S L C$ loops of the form

$$
\text { while }(B \mathbf{x} \leq \mathbf{0}) \text { do } \mathbf{x}^{\prime}=A^{\prime} \mathbf{x}+\mathbf{c}^{\prime}
$$

where the entries in $A^{\prime}$ and $\mathbf{c}^{\prime}$ are integer. 
Recall that a matrix $A$ is totally unimodular if each subdeterminant of $A$ is in $\{0, \pm 1\}$. In particular, the entries of such matrix are from $\{0, \pm 1\}$.

LEMMA 4.7. For any totally unimodular matrix $A$ and integer vector $\mathbf{b}$, the polyhedron $\mathcal{P}=\{\mathbf{x} \mid$ $A \mathbf{x} \leq \mathbf{b}\}$ is integral.

For brevity, if a polyhedron $\mathcal{P}$ is specified by $A \mathbf{x} \leq \mathbf{b}$ in which $A$ is a totally unimodular matrix and $\mathbf{b}$ an integer vector, we say that $\mathcal{P}$ is totally unimodular.

Corollary 4.8. The LiNRF $(\mathbb{Z})$ problem is PTIME-decidable for $S L C$ loops in which (1) the transition polyhedron $\mathcal{Q}$ is totally unimodular; or (2) the condition polyhedron $\mathcal{C}$ is totally unimodular and the update is linear with integer coefficients.

As a notable example, difference bound constraints Ben-Amram 2008; Bozga et al. 2012, Bozzelli and Pinchinat 2012 are defined by totally unimodular matrices. Such constraints have the form $x-y \leq d$ with $d \in \mathbb{Q}$; constraints of the form $\pm x \leq d$ can also be admitted. In the integer case we can always tighten $d$ to $\lfloor d\rfloor$ and thus get an integer polyhedron. It might be worth mentioning that checking if a matrix is totally unimodular can be done in polynomial time [Schrijver 1986. Th. 20.3, p. 290].

On the other hand, highlighting the gap between linear-ranking proofs and termination proofs in general, we may note that $M L C$ loops with difference bounds, even restricted to the forms $x_{i} \geq 0$ and $x_{i}^{\prime} \leq x_{j}+c$, already have an undecidable termination problem Ben-Amram 2008].

\subsection{Two-variable per inequality constraints}

In this section we consider cases in which the input loop involves two variable per inequality constraints ( TVPI for short), i.e., inequalities of the form $a x+b y \leq d$ with $a, b, d \in \mathbb{Q}$. Clearly, polyhedra defined by such inequalities are not guaranteed to be integral. See, for example, Figure 1(B).

Harvey [1999] showed that for two-dimensional polyhedra, which are specified by TVPI constraints by definition, the integer hull can be computed in $O\left(m \log A_{\text {max }}\right)$ where $m$ is the number of inequalities and $A_{\max }$ is the magnitude of the largest coefficient.

Definition 4.9. Let $T$ be a set of TVPI constraints. We say that $T$ is a product of independent two-dimensional TVPI constraints (PTVPI for short), if it can be partitioned into $T_{1}, \ldots, T_{n}$ such that (1) each $T_{i}$ is two-dimensional, i.e., involves at most two variables; and (2) each distinct $T_{i}$ and $T_{j}$ do not share variables.

LEMMA 4.10. The integer hull of PTVPI constraints can be computed in polynomial time.

Proof. Recall that a polyhedron $\mathcal{P}$ is integral if and only if each of its faces has an integer point. A face of $\mathcal{P}$ is obtained by turning some inequalities to equalities such that the resulting polyhedron in not empty (over the rationals). Clearly, if $T_{1}$ and $T_{2}$ are two sets of inequalities that do not share variables, and their faces have integer points, then all faces of $T_{1} \cup T_{2}$ have integer points. Thus, $T_{1} \cup T_{2}$ is integral. Partitioning $T$ into independent $T_{1}, \ldots, T_{n}$ and checking that each is 2-dimensional can done in polynomial time. Computing the integer hull of each $T_{i}$ can be done in polynomial time using Harvey's method.

The above approach can easily be generalized. Given any polyhedron, we first decompose it into independent sets of inequalities, in polynomial time (these are the connected components of an obvious graph), and then check if each set is covered by one of the special cases for which the integer hull can be efficiently computed. 
Corollary 4.11. The $\operatorname{LinRF}(\mathbb{Z})$ problem is PTIME-decidable for $S L C$ loops in which: (1) the transition polyhedron $\mathcal{Q}$ is PTVPI; or (2) the condition polyhedron $\mathcal{C}$ is PTVPI, and the update is linear with integer coefficients.

EXAMPLE 4.12. Consider the following $S L C$ loop, as an example for case (1) of Corollary 4.11

$$
\begin{aligned}
& \text { while }\left(4 x_{1} \geq 1, \quad x_{2} \geq 1\right) d o \\
& \qquad 2 x_{1}-5 x_{1}^{\prime} \leq 3, \quad-2 x_{1}+5 x_{1}^{\prime} \leq 1, \quad x_{2}^{\prime}=x_{2}+1
\end{aligned}
$$

Applying $\operatorname{LinRF}(\mathbb{Q})$ does not find a $L R F$ since the loop does not terminate when the variables range over $\mathbb{Q}$, e.g., for $x_{1}=\frac{1}{4}$ and $x_{2}=1$. The transition polyhedron is not integral, however, it is PTVPI since the constraints can be divided into $T_{1}=\left\{4 x_{1} \geq 1,2 x_{1}-5 x_{1}^{\prime} \leq 3,-2 x_{1}+5 x_{1}^{\prime} \leq 1\right\}$ and $T_{2}=\left\{x_{2} \geq 1, x_{2}^{\prime}=x_{2}+1\right\}$. It is easy to check that $T_{2}$ is already integral. Computing the integer hull of $T_{1}$ adds the inequalities $-x_{1}+x_{1}^{\prime} \leq-1$ and $\frac{1}{3} x_{1}-x_{1}^{\prime} \leq \frac{1}{3}$. See Figure $1(C)$. Now $\operatorname{LinRF}(\mathbb{Q})$ finds the $\operatorname{LRF} f\left(x_{1}, x_{2}\right)=x_{1}-1$.

EXAMPLE 4.13. Consider the following $S L C$ loop, as an example for case (2) of Corollary 4.11

$$
\begin{aligned}
& \text { while }\left(-x_{1}+x_{2} \leq 0, \quad-2 x_{1}-x_{2} \leq-1, \quad x_{3} \leq 1\right) d o \\
& \qquad x_{1}^{\prime}=x_{1}, \quad x_{2}^{\prime}=x_{2}-2 x_{1}+x_{3}, \quad x_{3}^{\prime}=x_{3}
\end{aligned}
$$

Applying $\operatorname{LinRF}(\mathbb{Q})$ does not find a $L R F$ since it does not terminate over $\mathbb{Q}$, e.g., for $x_{1}=x_{2}=\frac{1}{2}$ and $x_{3}=1$. The condition polyhedron is not integral, but it is PTVPI since the constraints can be divided into $T_{1}=\left\{-x_{1}+x_{2} \leq 0,-2 x_{1}-x_{2} \leq-1\right\}$ and $T_{2}=\left\{x_{3} \leq 1\right\}$. It is easily seen that $T_{2}$ is already integral; computing the integer hull of $T_{1}$ adds $x_{1} \geq 1$. See Figure $1(B)$. Now $\operatorname{LinRF}(\mathbb{Q})$ finds the $\operatorname{LRF} f\left(x_{1}, x_{2}, x_{3}\right)=2 x_{1}+x_{2}-1$. Note that the update in this loop involves constraints which are not TVPI.

\subsection{Octagonal relations}

TVPI constraints in which the coefficients are from $\{0, \pm 1\}$ have received considerable attention in the area of program analysis. Such constraints are called octagonal relations Miné 2006. A particular interest was in developing efficient algorithms for checking satisfiability of such relations, as well as inferring all implied octagonal inequalities, for variables ranging either over $\mathbb{Q}$ or over $\mathbb{Z}$.

Over $\mathbb{Q}$, this is done by computing the transitive closure of the relation, which basically adds inequalities that result from the addition of two existing inequalities, and possibly scaling to obtain coefficients of \pm 1 . For example: starting from the set of inequalities $\left\{-x_{1}+x_{2} \leq 0,-x_{1}-x_{2} \leq-1\right\}$, we add $-2 x_{1} \leq-1$, or, after scaling, $-x_{1} \leq-\frac{1}{2}$. Over $\mathbb{Z}$, this is done by computing the tight closure, which in addition to transitivity, is closed also under tightening. This operation replaces $a x+b y \leq d$ by $a x+b y \leq\lfloor d\rfloor$. For example, tightening $-x_{1} \leq-\frac{1}{2}$ yields $-x_{1} \leq-1$. The tight closure can be computed in polynomial time Bagnara et al. 2008a, Harvey and Stuckey 1997; Revesz 2009. Since the tightening eliminates some non-integer points, it is tempting to expect that it actually computes the integer hull. It is easy to show that this is true for two-dimensional relations, but it is false already in three dimensions, as we show in the following example.

EXAMPLE 4.14. Consider the following $S L C$ loop

$$
\begin{aligned}
& \text { while }\left(x_{1}+x_{2} \leq 2, \quad x_{1}+x_{3} \leq 3, \quad x_{2}+x_{3} \leq 4\right) d o \\
& x_{1}^{\prime}=1-x_{1}, \quad x_{2}^{\prime}=1+x_{1}, \quad x_{3}^{\prime}=1+x_{2}
\end{aligned}
$$


Note that the transition polyhedron is octagonal, but not integral. Applying $\operatorname{LinRF}(\mathbb{Q})$ does not find a $L R F$, since the loop does not terminate over $\mathbb{Q}$, e.g., for $x_{1}=\frac{1}{2}, x_{2}=\frac{3}{2}$, and $x_{3}=\frac{5}{2}$. Computing the tight closure does not change the transition (or condition) polyhedron, and thus, it is of no help in finding the $L R F$. In order to obtain the integer hull of the transition (or condition) polyhedron we should add $x_{1}+x_{2}+x_{3} \leq 4$, which is not an octagonal inequality. Having done so, $\operatorname{LinRF}(\mathbb{Q})$ finds the $\operatorname{LRF} f\left(x_{1}, x_{2}, x_{3}\right)=-3 x_{1}-4 x_{2}-2 x_{3}+12$.

Although it is not guaranteed that the tight closure of an octagonal relation corresponds to its integer hull, in practice, it does in many cases. Thus, since it can be computed in polynomial time, we suggest computing it before applying $\operatorname{LinRF}(\mathbb{Q})$ on loops that involve such relations. The above example shows that this does not give us a complete polynomial-time algorithm for $\operatorname{LinRF}(\mathbb{Z})$ over octagonal relations.

EXAMPLE 4.15. Consider Loop (1) of Section 1 in which the condition is an octagonal relation. $\operatorname{LinRF}(\mathbb{Q})$ fails to find a $L R F$ since the loop may fail to terminate for rational-valued variables. Computing the tight closure of the condition polyhedron adds the inequality $x \geq 1$, making the polyhedron integral. See Figure $1(\mathrm{~A})$. Now $\operatorname{LinRF}(\mathbb{Q})$ finds the $\operatorname{LRF} f\left(x_{1}, x_{2}\right)=x_{1}+x_{2}-1$. Let us consider an example with higher dimensions

$$
\begin{aligned}
& \text { while }\left(-x_{1}+x_{2} \leq 0, \quad-x_{1}-x_{2} \leq-1, \quad x_{2}-x_{3} \leq 0, \quad-x_{2}-x_{3} \leq-1\right) d o \\
& \quad x_{1}^{\prime}=x_{1}, \quad x_{2}^{\prime}=x_{2}-x_{1}-x_{3}+1, \quad x_{3}^{\prime}=x_{3}
\end{aligned}
$$

The condition polyhedron is octagonal, but not integral; moreover, it is not PTVPI. $\operatorname{LinRF}(\mathbb{Q})$ does not find a $L R F$ (indeed the loop fails to terminate for $x_{1}=x_{2}=x_{3}=\frac{1}{2}$ ). Computing the tight closure of the condition adds $-x_{1} \leq-1$ and $-x_{3} \leq 0$, which results in the integer hull. Now $\operatorname{LinRF}(\mathbb{Q})$ finds the $\operatorname{LRF} f\left(x_{1}, x_{2}, x_{3}\right)=x_{1}+x_{2}-1$.

A polynomial-time algorithm for computing the integer hull of octagonal relations is, unfortunately, ruled out by examples of such relations whose integer hulls have exponentially many facets.

THEOREM 4.16. There is no polynomial-time algorithm for computing the integer hull of general octagonal relations.

Proof. We build an octagonal relation $\mathcal{O}$, such that the minimum number of inequalities required to describe its integer hull $\mathcal{O}_{I}$ is not polynomial in the number of inequalities in $\mathcal{O}$.

For a complete graph $K_{n}=\langle V, E\rangle$, we let $\mathcal{P}$ be defined by the set of inequalities $\left\{x_{e} \geq 0 \mid e \in\right.$ $E\} \cup\left\{\sum_{v \in e} x_{e} \leq 1 \mid v \in V\right\}$. Here every edge $e \in E$ has a corresponding variable $x_{e}$, and the notation $v \in e$ means that $v$ is a vertex of edge $e$. Note that $\mathcal{P}$ is not octagonal. It is well-known that $\mathcal{P}_{I}$, the matching polytope of $K_{n}$, has at least $\left(\begin{array}{l}n \\ 2\end{array}\right)+2^{n-1}$ facets [Schrijver 1986. Sec. 18.2, p. 251], and thus any set of inequalities that defines $\mathcal{P}_{I}$ must have at least the same number of inequalities. Now let $\mathcal{O}$ be defined by $\left\{x_{e} \geq 0 \mid e \in E\right\} \cup\left\{x_{e_{1}}+x_{e_{2}} \leq 1 \mid v \in e_{1}, v \in e_{2}\right\}$, which includes $n+n \cdot\left(\begin{array}{c}n-1 \\ 2\end{array}\right)$ octagonal inequalities. It is easy to see that the integer solutions of $\mathcal{P}$ and $\mathcal{O}$ are the same, and thus $\mathcal{P}_{I}=\mathcal{O}_{I}$. This means that any set of inequalities that define $\mathcal{O}_{I}$ must have at least $\left(\begin{array}{l}n \\ 2\end{array}\right)+2^{n-1}$ inequalities. Therefore, any algorithm that computes such a representation must add at least $\left(\begin{array}{c}n \\ 2\end{array}\right)+2^{n-1}-n-n \cdot\left(\begin{array}{c}n-1 \\ 2\end{array}\right)$ inequalities to $\mathcal{O}$, which is super-polynomial in the size of $\mathcal{O}$. Unsurprisingly, the tight closure of $\mathcal{O}$ does not yield its integer hull (it only adds $x_{e} \leq 1$ for each $x_{e}$ ).

Note that the above theorem does not rule out a polynomial-time algorithm for $\operatorname{LiNRF}(\mathbb{Z})$, for $S L C$ loops in which the transition polyhedron $\mathcal{Q}$ is octagonal, or where the condition polyhedron is 
octagonal and the update is linear with integer coefficients. It just rules out an algorithm that is based on computing the integer hull of the polyhedra. However, the coNP-hardness proof of Section 3.1 could be also carried out by a reduction from 3SAT that produces an $S L C$ loop where the condition is octagonal and the update is linear with integer coefficients - so at least for this class there is, presumably, no polynomial solution. We present this reduction next

THEOREM 4.17. The $\operatorname{LINRF}(\mathbb{Z})$ problem is strongly coNP-hard, even for deterministic SLC loops where the guard is octagonal.

Proof. We exhibit a polynomial-time reduction from 3SAT to the complement of LiNRF( $\mathbb{Z})$ (keeping all the numbers in the resulting instance polynomially bounded, to obtain strong coNP-hardness).

Consider a 3SAT instance given as a collection of $m$ clauses, $C_{1}, \ldots, C_{m}$, each clause $C_{i}$ consisting of three literals $L_{i}^{j} \in\left\{x_{1}, \ldots, x_{n}, \bar{x}_{1}, \ldots, \bar{x}_{n}\right\}$. We construct a loop over $4 m$ variables. Variable $x_{i j}$ corresponds to $L_{i}^{j}$. Variable $x_{i 0}$ is a control variable to ensure the satisfaction of clause $i$, as will be seen below. Let $C$ be the set of all conflicting pairs, that is, pairs $((i, j),(r, s))$ such that $L_{i}^{j}$ is the complement of $L_{r}^{s}$, and also pairs $\left((i, j),\left(i, j^{\prime}\right)\right)$ with $1 \leq j<j^{\prime} \leq 3$. The loop we construct is:

$$
\begin{array}{r}
\operatorname{while}\left(\bigwedge_{((i, j),(r, s)) \in C} x_{i j}+x_{r s} \leq 1\right) \wedge\left(\bigwedge_{1 \leq i \leq m, 0 \leq j \leq 3} 0 \leq x_{i j} \leq 1\right) \\
\quad \operatorname{do}\left(\bigwedge_{1 \leq i \leq m, 1 \leq j \leq 3} x_{i j}^{\prime}=x_{i j}\right) \wedge\left(\bigwedge_{1 \leq i \leq m} x_{i 0}^{\prime}=x_{i 0}+x_{i 1}+x_{i 2}+x_{i 3}-1\right)
\end{array}
$$

Suppose the formula is satisfiable. For every clause, choose a satisfied literal, and set the corresponding variable $x_{i j}$ to 1 ; let all other variables be zero. Observe that all the inequality constraints are fulfilled, and that the value of each $x_{i 0}^{\prime}$ does not change. Hence, the loop does not terminate, and does not have any ranking function, let alone a $L R F$.

Next, suppose the formula is unsatisfiable. An initial state for which the loop guard is enabled may be interpreted as a selection of non-conflicting literals. Since no such selection can satisfy all clauses, looking at the update of the $x_{i 0}$ variables, we see that some may stay unchanged, while some (and at least one) will decrease. It follows that $\sum_{i} x_{i 0}$ is a $L R F$.

\subsection{Strongly polynomial cases}

Polynomial-time algorithms for $\operatorname{LinRF}(\mathbb{Q})$ Alias et al. 2010, Mesnard and Serebrenik 2008, Podelski and Rybalchenko 2004 inherit their complexity from that of $L P$. While it is known that $L P$ can be solved by a polynomial-time algorithm, it is an open problem whether it has a strongly polynomial algorithm. Such an algorithm should perform a number of elementary arithmetic operations polynomial in the dimensions of the input matrix instead of its bit-size (which accounts for the size of the matrix entries), and such operations should be performed on numbers of size which is polynomial to the input bit-size. However, there are some cases for which $L P$ is known to have a strongly polynomial algorithm. We first use these cases to define classes of $S L C$ loops for which $\operatorname{LinRF}(\mathbb{Q})$ has a strongly polynomial algorithm, which we then use to show that $\operatorname{LINRF}(\mathbb{Z})$ has a strongly polynomial algorithm for some corresponding classes of $S L C$ loops. Our results are based on the following result by Tardos [1986] (quoting Schrijver 1986, p. 196]). 
THEOREM 4.18 (Tardos). There is an algorithm which solves a rational LP problem $\max \{\mathbf{c} \cdot \mathbf{x} \mid$ $A \mathbf{x} \leq \mathbf{b}\}$ with at most $P($ size $(A))$ elementary arithmetic operations on numbers of size polynomially bounded by size $(A, \mathbf{b}, \mathbf{c})$, for some polynomial $P$.

Note that the number of arithmetic operations required by the $L P$ algorithm only depends on the bit-size of $A$. Clearly, if we restrict the $L P$ problem to cases in which the bit-size of the entries of $A$ is bounded by a constant, then size $(A)$ depends only on its dimensions, and we get a strongly polynomial time algorithm. In particular we can state the following.

Corollary 4.19. There exists a strongly polynomial algorithm to solve an $L P \operatorname{problem~} \max \{\mathbf{c} \cdot \mathbf{x} \mid$ $A \mathbf{x} \leq \mathbf{b}\}$ where the entries of $A$ are $\{0, \pm 1, \pm 2\}$.

We can use this to show that $\operatorname{LinRF}(\mathbb{Q})$ can sometimes be implemented with strongly polynomial complexity. To do this, we use the Podelski-Rybalchenko formulation of the procedure Podelski and Rybalchenko 2004, slightly modified to require that the $L R F$ decreases at least by 1 instead of by some $\delta>0$ (this modification only affects $28 \mathrm{e}$ below; the right-hand side of the inequality is $-\delta$, so in their formulation the inequality was $\left.\vec{\eta} \cdot \mathbf{c}^{\prime \prime}<0\right)$.

THEOREM 4.20 (Podelski-Rybalchenko). Given an $S L C$ loop with a transition polyhedron $\mathcal{Q} \subseteq$ $\mathbb{Q}^{2 n}$, specified by $A^{\prime \prime} \mathbf{x}^{\prime \prime} \leq \mathbf{c}^{\prime \prime}$, let $A^{\prime \prime}=\left(A A^{\prime}\right)$ where each $A$ and $A^{\prime}$ has $n$ columns and $m$ rows each, and let $\vec{\mu}, \vec{\eta}$ be row vectors of different $m$ rational variables each. A LRF for $\mathcal{Q}$ exists if and only if there is a (rational) solution to the following set of constraints

$$
\begin{aligned}
\vec{\mu}, \vec{\eta} & \geq \mathbf{0}^{T}, \\
\vec{\mu} \cdot A^{\prime} & =\mathbf{0}^{T}, \\
(\vec{\mu}-\vec{\eta}) \cdot A & =\mathbf{0}^{T}, \\
\vec{\eta} \cdot\left(A+A^{\prime}\right) & =\mathbf{0}^{T}, \\
\vec{\eta} \cdot \mathbf{c}^{\prime \prime} & \leq-1 .
\end{aligned}
$$

THEOREM 4.21. The $\operatorname{LinRF}(\mathbb{Q})$ problem is decidable in strongly polynomial time for SLC loops specified by $A^{\prime \prime} \mathbf{x}^{\prime \prime} \leq \mathbf{c}^{\prime \prime}$ where the coefficients of $A^{\prime \prime}$ are from $\{0, \pm 1\}$.

Proof. First observe that, in Theroem 4.20, when the matrix $A^{\prime \prime}$ has only entries from $\{0, \pm 1\}$, then all coefficients in the constraints (28a 28d) are from $\{0, \pm 1, \pm 2\}$. Moreover, the number of inequalities and variables in $28 \mathrm{a} 28 \mathrm{~d})$ is polynomial in the dimensions of $A^{\prime \prime}$. Now let us modify the PodelskiRybalchenko procedure such that instead of testing for feasibility of the constraints 28a 28e, we consider the minimization of $\vec{\eta} \cdot \mathbf{c}^{\prime \prime}$ under the other constraints 28a 28d). Clearly, this answers the same question since: 28a 28e is feasible, if and only if the minimization problem is unbounded, or the minimum is negative. This brings the problem to the form required by Corollary 4.19 and yields our result.

Corollary 4.22. The $\operatorname{LinRF}(\mathbb{Z})$ problem is decidable in strongly polynomial time for $S L C$ loops, specified by $A^{\prime \prime} \mathbf{x}^{\prime \prime} \leq \mathbf{c}^{\prime \prime}$, that are covered by any of the special cases of sections 4.1 and 4.2 and the entries of $A^{\prime \prime}$ are from $\{0, \pm 1\}$.

Proof. In the cases of Section 4.1, the transition polyhedron is guaranteed to be integral. In the PTVPI case of Section 4.2, (1) the integer hull can be computed using Harvey's procedure, which is 
strongly polynomial in this case since the entries of $A$ are from $\{0, \pm 1\}$. This can be done also using the tight closure of 2-dimensional octagons; and (2) the TVPI constraints that we add when computing the integer hull have coefficients from $\{0, \pm 1\}$, and the number of such constraints is polynomially bounded by the number of the original inequalities. Thus, by Theorem 4.21, we can apply a strongly polynomial-time algorithm for $\operatorname{LinRF}(\mathbb{Q})$.

\subsection{Multipath loops}

It follows immediately from the definitions that a linear function $\rho$ is a $L R F$ for an $M L C$ loop with transition polyhedra $\mathcal{Q}_{1}, \ldots, \mathcal{Q}_{k}$ if and only if it is a $L R F$ for each $\mathcal{Q}_{i}$. Thus, if we have the set of $L R F s$ for each $\mathcal{Q}_{i}$, we can simply take the intersection and obtain the set of $L R F s$ for $\mathcal{Q}_{1}, \ldots, \mathcal{Q}_{k}$. In the Podelski-Rybalchenko procedure, the set of solutions for the inequalities 28a 28e defines the set of $L R F s$ for the corresponding $S L C$ loop as follows.

LEMMA 4.23. Given an $S L C$ loop with a transition polyhedron $\mathcal{Q}$, specified by $A^{\prime \prime} \mathbf{x}^{\prime \prime} \leq \mathbf{c}^{\prime \prime}$, let $\Gamma\left(\vec{\mu}, \vec{\eta}, A^{\prime \prime}, c^{\prime \prime}\right)$ be the conjunction of 28a 28e). Then, $\rho(\mathbf{x})=\vec{\lambda} \cdot \mathbf{x}+\lambda_{0}$ is a LRF for $\mathcal{Q}$ if and only if $\Gamma\left(\vec{\mu}, \vec{\eta}, A^{\prime \prime}, c^{\prime \prime}\right)$ has a solution such that $\vec{\lambda}=\vec{\eta} \cdot A^{\prime}$ and $\lambda_{0} \geq \vec{\mu} \cdot \mathbf{c}^{\prime \prime}$.

Next we show how to compute, using the above lemma, the intersection of sets of LRFs for several transition polyhedra, and thus obtain the set of $L R F s$ for a given $M L C$ loop (a very similar statement stated by [Cook et al. 2010, Lemma 3]).

LEMMA 4.24. Given an $M L C$ loop with transition polyhedra $\mathcal{Q}_{1}, \ldots, \mathcal{Q}_{k}$, each specified by $A_{i}^{\prime \prime} \mathbf{x}^{\prime \prime} \leq$ $\mathbf{c}_{i}^{\prime \prime}$, let $\Gamma\left(\vec{\mu}_{i}, \vec{\eta}_{i}, A_{i}^{\prime \prime}, c_{i}^{\prime \prime}\right)$ be the constraints 28a 28e) for the $i$-th path, and $\left(\lambda_{0}, \vec{\lambda}\right)$ be $n+1$ rational variables. Then there is a $L R F$ for $\mathcal{Q}_{1}, \ldots, \mathcal{Q}_{k}$ if and only if the following is feasible (over the rationals)

$$
\bigwedge_{i=1}^{k} \Gamma\left(\vec{\mu}_{i}, \vec{\eta}_{i}, A_{i}^{\prime \prime}, c_{i}^{\prime \prime}\right) \wedge \vec{\lambda}=\vec{\eta}_{i} \cdot A_{i}^{\prime} \wedge \lambda_{0} \geq \vec{\mu}_{i} \cdot \mathbf{c}_{i}^{\prime \prime}
$$

Moreover, the values of $\left(\lambda_{0}, \vec{\lambda}\right)$ in the solutions of $(29)$ define the set of all LRFs for $\mathcal{Q}_{1}, \ldots, \mathcal{Q}_{k}$.

Proof. Immediate by Lemma 4.23 , noting that for each $1 \leq i \leq k$ the constraints $\Gamma\left(\vec{\mu}_{i}, \vec{\eta}_{i}, A_{i}^{\prime \prime}, c_{i}^{\prime \prime}\right)$ uses different $\vec{\mu}_{i}$ and $\vec{\eta}_{i}$, while $\left(\lambda_{0}, \vec{\lambda}\right)$ are the same for all $i$.

Corollary 4.25. The $\operatorname{LinRF}(\mathbb{Q})$ problem for $M L C$ loops is PTIME-decidable.

Proof. The size of the set of inequalities $(29)$ is polynomial in the size of the input $M L C$ loop, and checking if it has a rational solution can be done in polynomial time.

Corollary 4.26. The $\operatorname{LinRF}(\mathbb{Z})$ problem for $M L C$ loops is PTIME-decidable when each path corresponds to one of the special cases, for $S L C$ loops, discussed in sections 4.1 and 4.2 .

Proof. Immediate, since if the transition polyhedra are integral, $\operatorname{LinRF}(\mathbb{Z})$ and $\operatorname{Lin} R F(\mathbb{Q})$ are equivalent.

EXAMPLE 4.27. Consider an $M L C$ loop with the following two paths: Loop (1) of Section 1 , and the loop of Example 4.12. Applying $\operatorname{LinRF}(\mathbb{Q}$ ) (as in Lemma 4.24) does not find a $L R F$ since both paths do not terminate when the variables range over $\mathbb{Q}$. If we first compute the integer hull of both paths, $\operatorname{LinRF}(\mathbb{Q})$ finds the $\operatorname{LRF} f\left(x_{1}, x_{2}\right)=3 x_{1}+x_{2}-2$. Note that the integer hull of the first path is computable in polynomial time since the condition is PTVPI and the update is linear with integer coefficients. That of the second path has been computed in Example 4.12 . 


\section{The Lexicographic-Linear Ranking Problem}

In this section we turn to the problems of finding a Lexicographic-Linear Ranking Function $(L L R F)$, or determining if one exist (as defined in Section 2.4). We study the complexity of both LEXLINRF $(\mathbb{Z})$ and LEXLinRF $(\mathbb{Q})$ and develop corresponding complete algorithms for synthesizing LLRFs (moreover, LLRFs of smallest dimension).

In Section 5.1 we consider the $\operatorname{LExLinRF}(\mathbb{Z})$ problem, and develop a synthesis algorithm which has exponential-time complexity in general, and polynomial-time complexity for the special cases of Section 4. We also provide sufficient and necessary conditions for the existence of a $L L R F$ which imply the completeness of our algorithm. These conditions are used in Section 5.2 to show that LexLinRF $(\mathbb{Z})$ is coNP-complete.

In Section 5.3 we consider the $\operatorname{LEx} \operatorname{LinRF}(\mathbb{Q})$ problem. We observe that applying the algorithm of Section 5.1, which is complete for the integer case, does not result in general in a $L L R F$ for a rational loop, but just what we call a weak $L L R F$. This is a $L L R F$ as in Definition 2.5 but changing (11) to $\Delta \rho\left(\mathbf{x}^{\prime \prime}\right)>0$. It is not immediate that a weak ranking function even implies termination, since $\Delta \rho\left(\mathbf{x}^{\prime \prime}\right)$ can be arbitrarily close to zero. However, we prove that it does, and in fact such a weak ranking function can be converted to a $L L R F$. This provides a complete polynomial-time algorithm for $\operatorname{LExLinRF}(\mathbb{Q})$ (which is also optimal with respect to the dimension).

In the rest of this section we assume an input $M L C$ loop specified by the transition polyhedra $\mathcal{Q}_{1}, \cdots, \mathcal{Q}_{k}$, where each $\mathcal{Q}_{i}$ is given as a system of inequalities $A \mathbf{x}^{\prime \prime} \leq \mathbf{c}_{i}^{\prime \prime}$. Since we handle $M L C$ loops, our results apply to $S L C$ loops as a special case; we would like to point out, however, that the coNP-hardness already applies to $S L C$ loops (Section 5.2), and that some interesting examples which demonstrate the advantage of LLRFs over LRFs use just $S L C$ loops (e.g., Example 2.6 on Page 8).

\subsection{A Complete Algorithm for $\operatorname{LexLinRF}(\mathbb{Z})$}

The basic building blocks for our LLRFs are non-trivial quasi-LRFs. These are similar to LRFs, except that $\Delta \rho\left(\mathbf{x}^{\prime \prime}\right)>0$ is not required to hold for all transitions, but rather for at least one.

Definition 5.1. We say that an affine linear function $\rho$ is a quasi- $L R F$ for $T \subseteq \mathbb{Q}^{2 n}$ if for every $\mathbf{x}^{\prime \prime} \in T$ the following holds:

$$
\begin{aligned}
\rho(\mathbf{x}) & \geq 0 \\
\Delta \rho\left(\mathbf{x}^{\prime \prime}\right) & \geq 0
\end{aligned}
$$

We say that it is non-trivial if, in addition, inequality (31) is strict, i.e., $\Delta \rho\left(\mathbf{x}^{\prime \prime}\right)>0$, for at least one $\mathbf{x}^{\prime \prime} \in T$.

We say that $\rho$ is a quasi- $L R F$ for a rational (respectively integer) loop if it is a quasi- $L R F$ for its transition polyhedra (respectively, their integer points).

EXAMPLE 5.2. Consider the $S L C$ loop (12) of Example 2.6 $\rho_{1}\left(x_{1}, x_{2}, x_{3}\right)=x_{2}$ is a non-trivial quasi-LRF; $\rho_{2}\left(x_{1}, x_{2}, x_{3}\right)=x_{1}$ is not because $\Delta \rho_{2}\left(\mathbf{x}^{\prime \prime}\right)>0$ does not hold for any transition; and $\rho_{3}\left(x_{1}, x_{2}, x_{3}\right)=x_{3}$ is not because $\rho_{3}(\mathbf{x})<0$ for $\mathbf{x}=(2,1,-1)$. Now consider the $M L C$ loop (3) of Section 1. $\rho_{4}\left(x_{1}, x_{2}\right)=x_{1}$ is a non-trivial quasi- $L R F$ for both paths of this loop; and $\rho_{5}\left(x_{1}, x_{2}\right)=x_{2}$ is not quasi- $L R F$ since $\Delta \rho_{5}\left(\mathbf{x}^{\prime \prime}\right) \geq 0$ does not hold for all transitions, e.g., it fails for $\mathbf{x}^{\prime \prime}=(2,2,1,3)$. Note that $\rho_{5}$ is a quasi- $L R F$ for the second path, but this is not enough. 
Our $L L R F$ synthesis algorithm is based on repeatedly finding non-trivial quasi- $L R F s$, and therefore we first focus on developing a complete algorithm for synthesizing non-trivial quasi- $L R F s$. The next lemma explains how to represent the space $\mathcal{S}$ of all quasi- $L R F s$, afterwards, we explain how to pick a non-trivial one, if possible, from this space.

LEMMA 5.3. Given $\mathcal{Q}_{1}, \ldots, \mathcal{Q}_{k}$, it is possible to build, in polynomial time, a set of inequalities $\mathcal{S}$ whose solutions define the coefficient vectors of all quasi-LRFs for the corresponding transitions $\mathcal{Q}_{1} \cup \cdots \cup \mathcal{Q}_{k}$.

Proof. Consider the constraints built by the Podelski-Rybalchenko procedure of Theorem 4.20, and change (28e) to $\vec{\eta} \cdot \mathbf{c}^{\prime \prime} \leq 0$. Then, these constraints describe the set of all quasi-LRFs for $\mathcal{Q}$, rather than LRFs. Using the construction of Lemma 4.24, with this change, we get a polyhedron $\mathcal{S}$ of dimension $n^{\prime}=n+1+\sum_{i=1}^{k} 2 m_{i}$ where $m_{i}$ is the number of inequalities in $\mathcal{Q}_{i}$. Assume the first $n+1$ components correspond to the coefficients $\left(\lambda_{0}, \vec{\lambda}\right)$ (and the rest correspond to $\vec{\mu}$ and $\vec{\eta}$ ), then any point $\left(\lambda_{0}, \vec{\lambda}, \vec{\mu}, \vec{\eta}\right) \in \mathcal{S}$ defines a quasi- $L R F \rho(\mathbf{x})=\vec{\lambda} \cdot \mathbf{x}+\lambda_{0}$ for $\mathcal{Q}_{1} \cup \cdots \cup \mathcal{Q}_{k}$.

The next lemma explains how to pick a non-trivial quasi- $L R F \rho$, if any, from $\mathcal{S}$. Moreover, it shows how to pick one such that $\Delta \rho$ is strict for as many transitions as possible, i.e., there is no other quasi-LRF $\rho^{\prime}$, and valid transition $\mathbf{x}^{\prime \prime}$, such that $\Delta \rho^{\prime}\left(\mathbf{x}^{\prime \prime}\right)>0$ and $\Delta \rho^{\prime}\left(\mathbf{x}^{\prime \prime}\right)=0$. We refer to such non-trivial quasi-LRFs as optimal. The importance of this optimal choice is in that it leads to an algorithm that synthesizes LLRFs of minimal dimension.

LEMMA 5.4. There is a polynomial-time algorithm that finds a non-trivial quasi-LRF $\rho$, if there is any, for $\mathcal{Q}_{1}, \ldots, \mathcal{Q}_{k}$; moreover, for any quasi-LRF $\rho^{\prime}$, and valid transition $\mathbf{x}^{\prime \prime}, \Delta \rho^{\prime}\left(\mathbf{x}^{\prime \prime}\right)>0 \Rightarrow$ $\Delta \rho^{\prime}\left(\mathbf{x}^{\prime \prime}\right)=0$.

Proof. The algorithm follows the following steps:

(a) Construct a polyhedron $\mathcal{S}$ of all quasi-LRFs as in Lemma 5.3

(b) If $\mathcal{S}=\emptyset$ return NonE, otherwise, pick $\left(\lambda_{0}, \vec{\lambda}, \vec{\mu}, \vec{\eta}\right)$ in the relative interior ${ }^{2}$ of $\mathcal{S}$;

(c) If $\max \left\{\vec{\lambda} \cdot\left(\mathbf{x}-\mathbf{x}^{\prime}\right) \mid \mathbf{x}^{\prime \prime} \in \mathcal{Q}_{i}\right\}>0$, for some $1 \leq i \leq k$, return $\rho(\mathbf{x})=\vec{\lambda} \cdot \mathbf{x}+\lambda_{0}$, otherwise return NONE.

When the above algorithm returns $\rho \neq$ NoNE, it is a non-trivial quasi- $L R F$ since it is a quasi- $L R F$, and the last step guarantees the existence of at least one $\mathbf{x}^{\prime \prime}$ for which $\Delta \rho\left(\mathbf{x}^{\prime \prime}\right)>0$. To show completeness of the above algorithm and optimality of $\rho$, it is enough to show that for any $\left(\lambda_{0}^{\prime}, \vec{\lambda}^{\prime}, \vec{\mu}^{\prime}, \vec{\eta}^{\prime}\right) \in \mathcal{S}$ and $\mathbf{z}^{\prime \prime} \in \mathcal{Q}_{1} \cup \cdots \cup \mathcal{Q}_{k}$, we have $\vec{\lambda} \cdot\left(\mathbf{z}-\mathbf{z}^{\prime}\right)=0 \Rightarrow \vec{\lambda}^{\prime} \cdot\left(\mathbf{z}-\mathbf{z}^{\prime}\right)=0$.

So, assume that $\vec{\lambda} \cdot\left(\mathbf{z}-\mathbf{z}^{\prime}\right)=0$. Define the hyperplane $\mathcal{H}=\left\{\left(\alpha_{0}, \vec{\alpha}, \vec{\beta}, \vec{\gamma}\right) \in \mathbb{Q}^{n^{\prime}} \mid \vec{\alpha} \cdot\left(\mathbf{z}-\mathbf{z}^{\prime}\right)=0\right\}$ where $\vec{\alpha}$ is a vector of dimension $n$, and $n^{\prime}$ is the dimension of $\mathcal{S}$. By assumption, $\left(\lambda_{0}, \vec{\lambda}, \vec{\mu}, \vec{\eta}\right) \in \mathcal{S} \cap \mathcal{H}$. Note that $\mathcal{S} \cap \mathcal{H}$ is a face of $\mathcal{S}$. If it equals to $\mathcal{S}$, then $\left(\lambda_{0}^{\prime}, \vec{\lambda}^{\prime}, \vec{\mu}^{\prime}, \vec{\eta}^{\prime}\right) \in \mathcal{H}$ and our claim holds. Otherwise, it is a proper face of $\mathcal{S}$. Since $\left(\lambda_{0}, \vec{\lambda}, \vec{\mu}, \vec{\eta}\right)$ was chosen from the relative interior of $\mathcal{S}$, we have $\vec{\lambda} \cdot\left(\mathbf{z}-\mathbf{z}^{\prime}\right)>0$, and again our claim holds.

To justify the polynomial-time complexity note that the first step is polynomial by Lemma 5.3 the second step can be done in polynomial time Schrijver 1986, Cor. 14.1g,p. 185]; and the third is also polynomial since it consists of solving at most $k L P$ problems over the rationals.

\footnotetext{
${ }^{2}$ For definitions related to faces of polyhedra, and the relative interior, see Section 2.1
} 


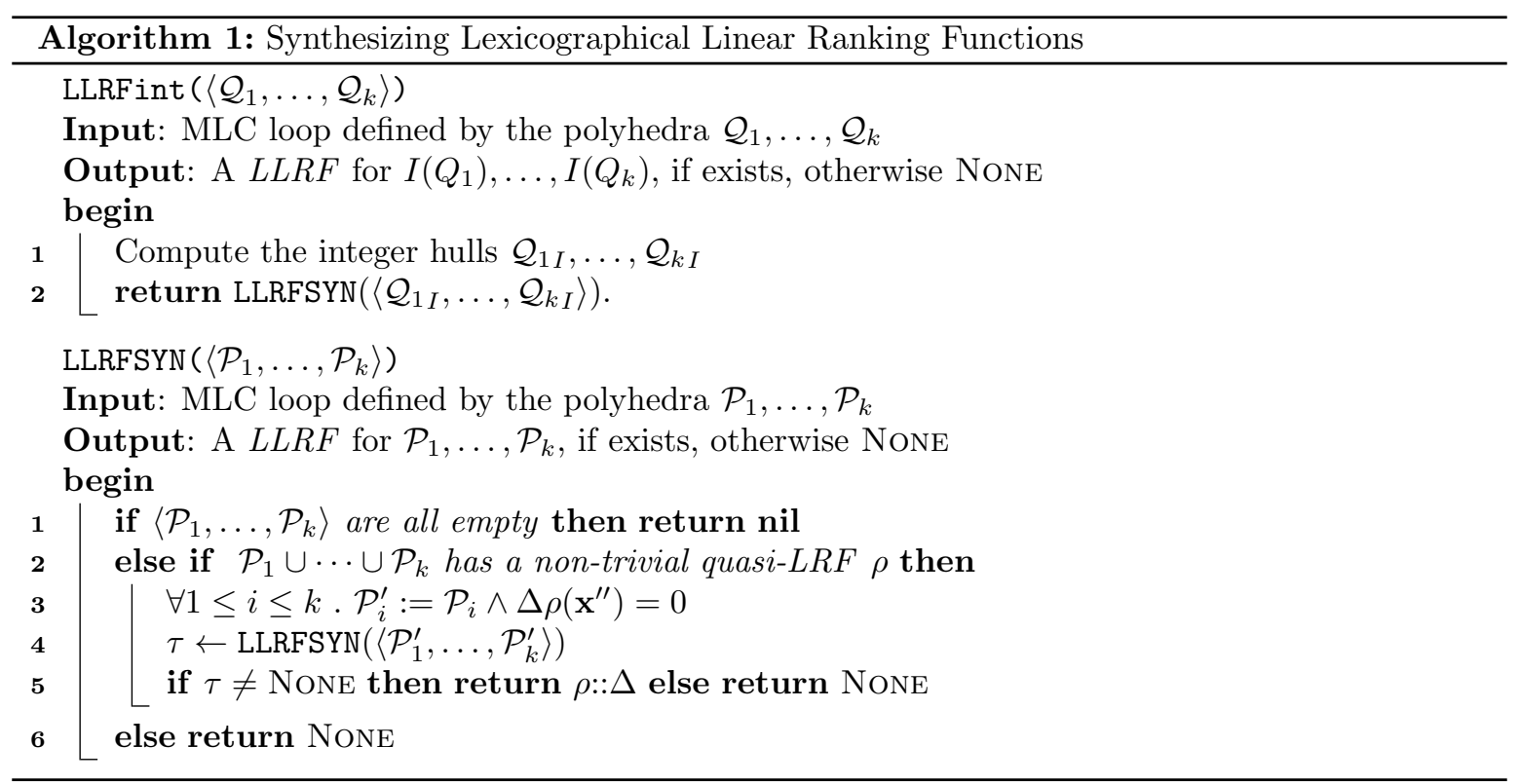

Next we observe that finding a non-trivial quasi- $L R F$ for $I\left(\mathcal{Q}_{1}\right) \cup \cdots \cup I\left(\mathcal{Q}_{k}\right)$, i.e., over the integers, can be done by finding one for the corresponding integer hulls.

LEMMA 5.5. Function $\rho$ a is non-trivial quasi-LRF for $I\left(\mathcal{Q}_{1}\right) \cup \cdots \cup I\left(\mathcal{Q}_{k}\right)$ if and only if it is a non-trivial quasi-LRF for $\mathcal{Q}_{1 I} \cup \cdots \cup \mathcal{Q}_{k_{I}}$.

Proof. $(\Rightarrow)$ Suppose $\rho$ is a non-trivial quasi- $L R F$ for $I\left(\mathcal{Q}_{1}\right) \cup \cdots \cup I\left(\mathcal{Q}_{k}\right)$. Then, since $I\left(\mathcal{Q}_{i}\right) \subseteq \mathcal{Q}_{i I}$, there is an integer point $\mathbf{x}^{\prime \prime} \in \mathcal{Q}_{1 I} \cup \cdots \cup \mathcal{Q}_{k I}$ for which $\Delta \rho\left(\mathbf{x}^{\prime \prime}\right)>0$. It remains to show that for any $\mathbf{x}^{\prime \prime} \in \mathcal{Q}_{1_{I}} \cup \cdots \cup \mathcal{Q}_{k_{I}}$ we have $\rho(\mathbf{x}) \geq 0$ and $\Delta \rho\left(\mathbf{x}^{\prime \prime}\right) \geq 0$. This follows from the fact that, by definition of integer polyhedra, any $\mathbf{x}^{\prime \prime} \in \mathcal{Q}_{i_{I}}$ is a convex combination of some points from $I\left(\mathcal{Q}_{i}\right) .(\Leftarrow)$ Suppose $\rho$ is a non-trivial quasi- $L R F$ for $\mathcal{Q}_{1 I} \cup \cdots \cup \mathcal{Q}_{k_{I}}$. Then, for any $\mathbf{x}^{\prime \prime} \in I\left(\mathcal{Q}_{1}\right) \cup \cdots \cup I\left(\mathcal{Q}_{k}\right)$ we have $\rho(\mathbf{x}) \geq 0$ and $\Delta \rho\left(\mathbf{x}^{\prime \prime}\right) \geq 0$. It remains to show that there is $\mathbf{x}^{\prime \prime} \in I\left(\mathcal{Q}_{1}\right) \cup \cdots \cup I\left(\mathcal{Q}_{k}\right)$ for which $\Delta \rho\left(\mathbf{x}^{\prime \prime}\right)>0$. Let $\mathbf{x}^{\prime \prime} \in \mathcal{Q}_{i I}$ be a point for which $\Delta \rho\left(\mathbf{x}^{\prime \prime}\right)>0$, then, since $\mathbf{x}^{\prime \prime}$ is a convex combination of some integer points from $I\left(\mathcal{Q}_{i}\right)$, there must be an integer point $\mathbf{z}^{\prime \prime} \in I\left(\mathcal{Q}_{i}\right)$ for which $\Delta \rho\left(\mathbf{z}^{\prime \prime}\right)>0$.

LEMMA 5.6. If there is a non-trivial quasi-LRF for $I\left(\mathcal{Q}_{1}\right) \cup \cdots \cup I\left(\mathcal{Q}_{k}\right)$, then there is one such that for any $\mathbf{x}^{\prime \prime} \in I\left(\mathcal{Q}_{1}\right) \cup \cdots \cup I\left(\mathcal{Q}_{k}\right)$, either $\Delta \rho\left(\mathbf{x}^{\prime \prime}\right)=0$ or $\Delta \rho\left(\mathbf{x}^{\prime \prime}\right) \geq 1$.

Proof. We compute any non-trivial quasi- $L R F$, and multiply it by an integer big enough to make its coefficients integer (e.g., by the least common multiplier of the denominators of its rational coefficients). The co-domain of the resulting function is $\mathbb{Z}\left(\right.$ since the domain is $\left.\mathbb{Z}^{n}\right)$.

Note that the above Lemma does not hold when considering non-trivial quasi-LRFs for $\mathcal{Q}_{1} \cup \cdots \cup \mathcal{Q}_{k}$, i.e., over the rationals. As we will see in Section 5.3, this is the main obstacle when trying to use the techniques of this section for $\operatorname{LExLinRF}(\mathbb{Q})$.

Now we are in a position for describing our algorithm for synthesizing a $L L R F$, shown as the procedure LLRFint in Algorithm 1. It either returns a $L L R F \tau$ or NoNE if none exists. Let us 
first explain the recursive procedure LLRFSYN. It builds the $L L R F$ component by component, or more precisely, by finding a suitable first component and calling itself recursively to find the rest. Specifically, at Line 2 it finds a non-trivial quasi- $L R F \rho$ for the transitions $\mathcal{P}_{1} \cup \cdots \cup \mathcal{P}_{k}$. Assuming it is as in Lemma 5.6. then this $\rho$ ranks all transitions for which $\Delta \rho\left(\mathbf{x}^{\prime \prime}\right) \geq 1$. The other transitions, i.e., those for which $\Delta \rho\left(\mathbf{x}^{\prime \prime}\right)=0$, are computed at Line 3 , and at Line 4 LLRFSYN is recursively called in order to find a $L L R F \tau$ for them. If it finds one, then it returns $\rho:: \tau$ as a $L L R F$ for $\mathcal{P}_{1} \cup \cdots \cup \mathcal{P}_{k}$. The recursion stops when all transitions are ranked (Line 1), or when there is no non-trivial quasi- $L R F$ for the current set of transitions (Line 3). An important property of this algorithm is that when calling LLRFSYN with integral polyhedra, then the polyhedra passed to the recursive call are also integral. This allows us to rely on Lemmas 5.4 and 5.5, which entail the completeness of the overall algorithm. This also explains why it suffices to compute the integer hulls once, at Line 1 of Procedure LLRFint.

EXAMPLE 5.7. Let us demonstrate the algorithm on the $S L C$ loop 12 of Example 2.6, which is defined by

$$
\mathcal{Q}=\left\{x_{1} \geq 0, x_{2} \geq 0, x_{3} \geq-x_{1}, x_{2}^{\prime}=x_{2}-x_{1}, x_{3}^{\prime}=x_{3}+x_{1}-1\right\} .
$$

First note that in this case $\mathcal{Q}_{I}=\mathcal{Q}$ and thus we can skip Line 1 of Procedure LLRFint. LLRFSYN is first called with $\mathcal{Q}$, and then, at Line 2 it finds the non-trivial quasi- $L R F \rho_{1}\left(x_{1}, x_{2}, x_{3}\right)=x_{2}$ for $\mathcal{Q}$, at Line 3 it sets $\mathcal{P}_{1}^{\prime}$ to $\mathcal{Q} \wedge x_{2}-x_{2}^{\prime}=0$, and at Line 4 LLRFSYN is called recursively with this $\mathcal{P}_{1}^{\prime}$. Then, at Line 2 it finds the non-trivial quasi- $\operatorname{LRF} \rho_{2}\left(x_{1}, x_{2}, x_{3}\right)=x_{3}$ for $\mathcal{Q} \wedge x_{2}-x_{2}^{\prime}=0$, at Line 3 it sets $\mathcal{P}_{1}^{\prime}$ to $\mathcal{Q} \wedge x_{2}-x_{2}^{\prime}=0 \wedge x_{3}-x_{3}^{\prime}=0$ which is an empty polyhedron, and at Line 4 LLRFSYN is called recursively with an empty polyhedron. Then, the check at Line 1 succeeds and it returns nil. Thus, the final returned value is $\left\langle x_{2}, x_{3}\right\rangle$ which is a $L L R F$ for $I\left(\mathcal{Q}_{1}\right)$. Now suppose that we remove $x_{3} \geq-x_{1}$ from $\mathcal{Q}$, and note that we still have $\mathcal{Q}_{I}=\mathcal{Q}$. Calling LLRFSYN with this modified $\mathcal{Q}$ would proceeds as above, however, it would fail to find a non-trivial quasi- $L R F$ for $\mathcal{Q} \wedge x_{2}-x_{2}^{\prime}=0$ and thus it returns None. Indeed, in this case $I(\mathcal{Q})$ does not have a $L L R F$ since the loop is non-terminating.

Before formally proving soundness and completeness of Algorithm 1 we state a fundamental observation that we will rely on.

Observation 5.8. Let $\mathcal{Q}$ be a transition polyhedron. If $\rho$ is a quasi- $L R F$ for $\mathcal{Q}$, then the points where $\Delta \rho\left(\mathbf{x}^{\prime \prime}\right)=0$ holds, if any, form a face of $\mathcal{Q}$.

Proof. If there is $\mathbf{x}^{\prime \prime} \in \mathcal{Q}$ such that $\Delta \rho\left(\mathbf{x}^{\prime \prime}\right)=0$, then $\min \left\{\Delta \rho\left(\mathbf{x}^{\prime \prime}\right) \mid \mathbf{x}^{\prime \prime} \in \mathcal{Q}\right\}=0$. According to the definition of a face, the intersection of the hyperplane $\left\{\mathbf{x}^{\prime \prime} \in \mathbb{Q}^{2 n} \mid \Delta \rho\left(\mathbf{x}^{\prime \prime}\right)=0\right\}$ with $\mathcal{Q}$ is a face of $\mathcal{Q}$.

Note that the statement that $\rho$ is non-trivial is equivalent to stating that the face, above, is proper.

LEMMA 5.9. If LLRFint $\left(\left\langle\mathcal{Q}_{1}, \ldots, \mathcal{Q}_{k}\right\rangle\right)$ returns $\tau$ different from NonE, then $\tau$ is a LLRF for $I\left(\mathcal{Q}_{1}\right), \ldots, I\left(\mathcal{Q}_{k}\right)$.

Proof. We show that when $\mathcal{P}_{1}, \ldots, \mathcal{P}_{k}$ are integral, and $\operatorname{LLRFSYN}\left(\left\langle\mathcal{P}_{1}, \ldots, \mathcal{P}_{k}\right\rangle\right)$ returns $\tau \neq$ None, then $\tau$ is a $L L R F$ for $I\left(\mathcal{P}_{1}\right), \ldots, I\left(P_{k}\right)$. The conclusion of the lemma then follows because LLRFint calls LLRFSYN with the integer polyhedra $\mathcal{Q}_{1 I}, \ldots, \mathcal{Q}_{k I}$. The proof is by induction on $\sum \operatorname{dim}\left(\mathcal{P}_{i}\right)$.

Base-case. The base-case is when $\sum \operatorname{dim}\left(\mathcal{P}_{i}\right)=-k$, i.e., all $\mathcal{P}_{i}$ are empty. In such case the algorithm returns nil, which is trivially correct since there are no transitions. 
Induction hypothesis. If $\sum \operatorname{dim}\left(\mathcal{P}_{i}\right)<j$, each $\mathcal{P}_{i}$ is integral, and $\operatorname{LLRFSYN}\left(\left\langle\mathcal{P}_{1}, \ldots, \mathcal{P}_{k}\right\rangle\right)$ returns $\tau$, then $\tau$ is a $L L R F$ for $I\left(\mathcal{P}_{1}\right), \ldots, I\left(\mathcal{P}_{k}\right)$.

Induction step. Assume $\sum \operatorname{dim}\left(\mathcal{P}_{i}\right)=j$, and that $\operatorname{LLRFSYN}\left(\left\langle\mathcal{P}_{1}, \ldots, \mathcal{P}_{k}\right\rangle\right)$ returns $\rho:: \tau$. Namely, at Line 2 it finds $\rho$, and $\tau \neq$ None is the result of $\operatorname{LLRFSYN}\left(\left\langle\mathcal{P}_{1}^{\prime}, \ldots, \mathcal{P}_{k}^{\prime}\right\rangle\right)$ at Line 4 . We show that $\rho:: \tau$ is a $L L R F$ for $I\left(\mathcal{P}_{1}\right), \ldots, I\left(\mathcal{P}_{k}\right)$. First note the following:

1. Each $\mathcal{P}_{i}^{\prime}$ is integral. This is because it is either empty, or a face of $\mathcal{P}_{i}$ (by Lemma 5.8), and all faces of an integral polyhedron are integral.

2. $\sum \operatorname{dim}\left(\mathcal{P}_{i}^{\prime}\right)<\sum \operatorname{dim}\left(\mathcal{P}_{i}\right)=j$. This is because $(i) \forall 1 \leq i \leq k . \operatorname{dim}\left(\mathcal{P}_{i}^{\prime}\right) \leq \operatorname{dim}\left(\mathcal{P}_{i}\right) ;$ and $(i i)$ there is $\mathbf{x}^{\prime \prime} \in \mathcal{P}_{i}$, for some $i$, such that $\Delta \rho\left(\mathbf{x}^{\prime \prime}\right)>0$, and thus $\mathcal{P}_{i}^{\prime}$ is either empty or a proper face of $\mathcal{P}_{i}$ (by Lemma 5.8), in both cases $\operatorname{dim}\left(\mathcal{P}_{i}^{\prime}\right)<\operatorname{dim}\left(\mathcal{P}_{i}\right)$.

3. By Lemma 5.6. we may assume that the function $\rho$ has been scaled, if necessary, so that for any $\mathbf{x}^{\prime \prime} \in I\left(\mathcal{P}_{1}\right) \cup \cdots \cup I\left(\mathcal{P}_{k}\right)$, either $\Delta \rho\left(\mathbf{x}^{\prime \prime}\right)=0$ and $\mathbf{x}^{\prime \prime} \in I\left(\mathcal{P}_{1}^{\prime}\right) \cup \cdots \cup I\left(\mathcal{P}_{k}^{\prime}\right)$, or $\Delta \rho\left(\mathbf{x}^{\prime \prime}\right) \geq 1$.

Using (12), we apply the induction hypothesis and conclude that $\tau$ is a $L L R F$ for $I\left(\mathcal{P}_{1}^{\prime}\right), \ldots, I\left(\mathcal{P}_{k}^{\prime}\right)$. Using (3) we conclude that $\rho:: \tau$ is still a $L L R F$ for $I\left(\mathcal{P}_{1}^{\prime}\right), \ldots, I\left(\mathcal{P}_{k}^{\prime}\right)$, and that $\rho$ ranks all transitions of $I\left(\mathcal{P}_{1}\right) \cup \cdots \cup I\left(\mathcal{P}_{k}\right)$ that are not in $I\left(\mathcal{P}_{1}^{\prime}\right) \cup \cdots \cup I\left(\mathcal{P}_{k}^{\prime}\right)$. Thus, $\rho:: \tau$ is a $L L R F$ for $I\left(\mathcal{P}_{1}\right), \ldots, I\left(\mathcal{P}_{k}\right)$.

Lemma 5.9 proves that Algorithm 1 is a sound procedure for $\operatorname{LexLinRF}(\mathbb{Z})$. In Theorem 5.12 below we combine this with a completeness proof. First, we give sufficient and necessary conditions for the existence of a $L L R F$ for $I\left(\mathcal{Q}_{1}\right), \ldots, I\left(\mathcal{Q}_{k}\right)$.

Observation 5.10. If there is a $L L R F$ for $I\left(\mathcal{Q}_{1}\right), \ldots, I\left(\mathcal{Q}_{k}\right)$, then every set of transitions $T \subseteq I\left(\mathcal{Q}_{1}\right) \cup$ $\cdots \cup I\left(\mathcal{Q}_{k}\right)$ has a non-trivial quasi- $L R F$.

Proof. Let $\tau=\left\langle\rho_{1}, \ldots, \rho_{d}\right\rangle$ be a $L L R F$ for $I\left(\mathcal{Q}_{1}\right), \ldots, I\left(\mathcal{Q}_{k}\right)$, and $T$ be a set of transitions. Define $I=\left\{i \mid \mathbf{x}^{\prime \prime} \in T\right.$ is ranked by $\left.\rho_{i}\right\}$, and let $j=\min (I)$. Then, from Definition 2.5, it is easy to verify that $\rho_{j}$ is a non-trivial quasi- $L R F$ for $T$.

Observation 5.11. If every set of transitions $T \subseteq I\left(\mathcal{Q}_{1}\right) \cup \cdots \cup I\left(\mathcal{Q}_{k}\right)$ has a non-trivial quasi- $L R F$, then there is a $L L R F$ for $I\left(\mathcal{Q}_{1}\right), \ldots, I\left(\mathcal{Q}_{k}\right)$.

Proof. In such case, Algorithm1, will find a $L L R F$. This is because in every call to LLRFSYN, $\mathcal{P}_{1}, \ldots, \mathcal{P}_{k}$ are integral, and thus, by Lemmas 5.4 and 5.5 the check at Line 2 of LLRFSYN is complete. Moreover, the algorithm terminates since $\sum \operatorname{dim}\left(\mathcal{P}_{i}\right)$ decreases in each recursive call and has a lower bound $-k$.

THEOREM 5.12. Algorithm 1 is sound and complete for $\operatorname{LEXLinRF}(\mathbb{Z})$. Moreover, when it finds a LLRF, it finds one of a minimal dimension.

Proof. If the algorithm returns $\tau=\left\langle\rho_{1}, \ldots, \rho_{d}\right\rangle$, then, by Lemma 5.9 it is a $L L R F$. If it is returns None, then it has found a subset of integer points (at Line 2 of LLRFSYN) that does not have a non-trivial quasi- $L R F$. In this case, by Observation 5.10, there is no $L L R F$. Thus, soundness and completeness have been established.

The minimality of the dimension stems from the fact that our algorithm is greedy, i.e., in each step finds (by Lemma 5.4) a $L L R F$ that ranks as many transitions as possible. Assume there is another LLRF $\tau^{\prime}=\left\langle\rho_{1}^{\prime}, \ldots, \rho_{d^{\prime}}^{\prime}\right\rangle$. We show by induction that the set of transitions that are not ranked by 
$\left\langle\rho_{1}, \ldots, \rho_{i}\right\rangle$, call it $\mathcal{U}_{i}$, is contained in the set of transitions not ranked by $\left\langle\rho_{1}^{\prime}, \ldots, \rho_{i}^{\prime}\right\rangle$, call them $\mathcal{U}_{i}^{\prime}$. Observe that since LLRFSYN returns immediately if the input polyhedra are empty, we must have $\mathcal{U}_{i} \neq \emptyset$ for $i \leq d$. It follows that $\mathcal{U}_{i}^{\prime} \neq \emptyset$ for $i \leq d$, hence $d^{\prime} \geq d$.

The claim holds by definition for $i=0$ since $\mathcal{U}_{0}=\mathcal{U}_{0}^{\prime}=I\left(\mathcal{Q}_{1}\right) \cup \cdots \cup I\left(\mathcal{Q}_{1}\right)$. Assume $\mathcal{U}_{i} \subseteq \mathcal{U}_{i}^{\prime}$ for some $0 \leq i<d^{\prime}$, we show that $\mathcal{U}_{i+1} \subseteq \mathcal{U}_{i+1}^{\prime}$. Since $\mathcal{U}_{i} \subseteq \mathcal{U}_{i}^{\prime}$ then $\rho_{i+1}^{\prime}$ is a quasi- $L R F$ for $\mathcal{U}_{i}$, and since $\rho_{i+1}$ is optimal for $\mathcal{U}_{i}$, by Lemma 5.4 it cannot be that $\rho_{i+1}^{\prime}$ ranks a transition from $\mathcal{U}_{i}$ that is not ranked by $\rho_{i+1}$, thus $\mathcal{U}_{i+1} \subseteq \mathcal{U}_{i+1}^{\prime}$.

The next corollary bounds the dimension of the LLRF inferred by LLRFSYN in terms of $n$, the number of variables in the loop.

Corollary 5.13. If LLRFSYN returns $\tau=\left\langle\rho_{1}, \ldots, \rho_{d}\right\rangle$, then $d \leq n$.

Proof. Let $\vec{\lambda}_{i}$ be the coefficients of $\rho_{i}$ (i.e., we ignore the constant $\lambda_{0}$ ); for $1 \leq i \leq d$. We claim that

the vectors $\vec{\lambda}_{i}$ must be linearly independent. Assume the contrary; let $i$ be the first index such that $\vec{\lambda}_{i}=c_{1} \cdot \vec{\lambda}_{1}+\cdots+c_{i-1} \cdot \vec{\lambda}_{i-1}$. Pick a transition $\mathbf{x}^{\prime \prime}$ that is ranked by $\rho_{i}$, i.e., $\Delta \rho_{i}\left(\mathbf{x}^{\prime \prime}\right)=\vec{\lambda}_{i} \cdot\left(\mathbf{x}-\mathbf{x}^{\prime}\right)>0$ and $\forall 1 \leq j<i . \Delta \rho_{j}\left(\mathbf{x}^{\prime \prime}\right)=\vec{\lambda}_{j} \cdot\left(\mathbf{x}-\mathbf{x}^{\prime}\right)=0$. Then

$$
\Delta \rho_{i}\left(\mathbf{x}^{\prime \prime}\right)=\vec{\lambda}_{i} \cdot\left(\mathbf{x}-\mathbf{x}^{\prime}\right)=\left(\sum_{j=1}^{i-1} c_{j} \cdot \vec{\lambda}_{j}\right) \cdot\left(\mathbf{x}-\mathbf{x}^{\prime}\right)=\sum_{j=1}^{i-1} c_{j} \cdot \vec{\lambda}_{j} \cdot\left(\mathbf{x}-\mathbf{x}^{\prime}\right)=0
$$

which contradicts the assumption that $\Delta \rho_{i}\left(\mathbf{x}^{\prime \prime}\right)>0$. Now since each $\vec{\lambda}_{i}$ is a vector in $\mathbb{Q}^{n}$, linear independence implies $d \leq n$.

The above Lemma provides the best bound possible for $M L C$ loops. To see this, consider the $M L C$ loop (3) of Section 1. for which $n=2$, and note that it has a $L L R F$ with $d=2$, namely $\left\langle x_{1}, x_{2}\right\rangle$, but no $L L R F$ with $d=1$ (since it does not have a $L R F$ ). This can easily be extended to provide an example for any $n$.

Next, we argue that Procedure LLRFSYN can be implemented in polynomial time. Note that this does not mean that $\operatorname{LExLinRF}(\mathbb{Z})$ is PTIME-decidable since Algorithm 1 has to compute the integer hulls first, which may take exponential time. However, this does mean that in certain special cases, $\operatorname{LExLinRF}(\mathbb{Z})$ is PTIME-decidable.

LEMMA 5.14. Procedure LLRFSYN can be implemented in polynomial time.

Proof. First note that by Corollary 5.13 the recursion depth is bounded by $n+1$, and that lines 1 and 2 can be performed in polynomial time in the bit-size of (the current) $\mathcal{P}_{1}, \ldots, \mathcal{P}_{n}$. However, we cannot immediately conclude that the overall runtime is polynomial since as recursion progresses, the procedure operates on polyhedra obtained by adding additional constraints (at Line 3), that could get bigger and bigger in their bit-size. Thus, to complete the proof, we need to ensure that the bit-size of $\mathcal{P}_{1}, \ldots, \mathcal{P}_{n}$, at any stage of the recursion, is polynomial in the bit-size of the original ones. Next we show how Line 3 can be implemented to ensure this, exploiting the fact that when $\mathcal{P}_{i} \wedge \Delta \rho\left(\mathbf{x}^{\prime \prime}\right)=0$ is not empty, it is a face of $\mathcal{P}_{i}$.

Recall that any face of $\mathcal{P}_{i}$ can be obtained by changing some of its inequalities to equalities. Hence, instead of adding $\Delta \rho\left(\mathbf{x}^{\prime \prime}\right)=0$ to $\mathcal{P}_{i}$ at Line 3, we can identify those inequalities of $\mathcal{P}_{i}$ that should be turned into equalities to get $\mathcal{P}_{i} \wedge \Delta \rho\left(\mathbf{x}^{\prime \prime}\right)=0$. Changing these inequalities to equalities ensures that the bit-size of $\mathcal{P}_{i}$, at any stage of the recursion, is at most twice its original bit-size. Finding these inequalities can be done as follows: for each inequality $\mathbf{a} \cdot \mathbf{x} \leq b$ of $\mathcal{P}_{i}$, we check if 
$\mathcal{P}_{i} \wedge \Delta \rho\left(\mathbf{x}^{\prime \prime}\right)=0 \Rightarrow \mathbf{a} \cdot \mathbf{x} \geq b$ holds, if so, then this inequality should be turned to equality. This check can be done in polynomial time since it is an $L P$ problem and the bit-size of $\rho$ is polynomial in the bit-size of $\mathcal{P}_{1}, \ldots, \mathcal{P}_{n}$.

The above lemma implies that, as for $\operatorname{LinRF}(\mathbb{Z})$, if it is guaranteed that the transition polyhedra are integral, or their integer hull can be computed in polynomial time, then the $\operatorname{LExLiNRF}(\mathbb{Z})$ problem can be solved in polynomial time.

THEOREM 5.15. The LEXLinRF $(\mathbb{Z})$ problem for $M L C$ loops is PTIME-decidable if each path corresponds to one of the special cases discussed in sections 4.1 and 4.2.

Proof. For those special cases either we do not compute the integer hulls since they are already integral, or we compute them in polynomial time. Then Algorithm 1 becomes polynomial-time since Line 1 of LLRFint can be done in polynomial time, and LLRFSYN is polynomial according to Lemma 5.14.

It may be worthwhile to point out that even if we do not have a PTIME-decidable case, we can always apply Procedure LLRFSYN to the given polyhedra - if it produces a $L L R F$, we have a sound result in polynomial time.

\subsection{Complexity of $\operatorname{Lex} \operatorname{LinRF}(\mathbb{Z})$}

In this section we show that the $\operatorname{LExLinRF}(\mathbb{Z})$ problem, in the general case, is coNP-complete. First, coNP-hardness follows from the coNP-hardness of $\operatorname{LinRF}(\mathbb{Z})$ as in Theorem 3.1. This is because the construction in Theorem 3.1 either produces a loop that has a $L R F$ (which is also a $L L R F$ ) or else it is non-terminating (so it does not have any kind of ranking function). For the inclusion in coNP, we show that the complement problem, i.e., the nonexistence of a $L L R F$, has a polynomially checkable witness.

Corollary 5.16. There is no $L L R F$ for $I\left(\mathcal{Q}_{1}\right), \ldots, I\left(\mathcal{Q}_{k}\right)$, if and only if there is $T \subseteq I\left(\mathcal{Q}_{1}\right) \cup \cdots \cup I\left(\mathcal{Q}_{k}\right)$ for which there is no non-trivial quasi- $L R F$.

Proof. Immediate from Observations 5.10 and 5.11

The above observation suggests that such $T$ can be used as a witness, however, $T$ might include infinite number of transitions, and thus it does not immediately meet our needs (polynomially checkable witness).

EXAMPLE 5.17. We show a case in which $T$ must consist of infinitely many points. Let $\mathcal{Q}=\left\{x^{\prime} \leq\right.$ $x-1\}$ and take an arbitrary finite $T \subseteq \mathcal{Q}$. Now define $\lambda_{0}=\min \left\{x \mid\left(x, x^{\prime}\right) \in T\right\}$, then $\rho(x)=x-\lambda_{0}$ is a non-trivial quasi- $L R F$ (actually $L R F$ ) for $T$ and thus $T$ does not prove that there is no quasi- $L R F$ for $\mathcal{Q}$. Any set of transitions out of $\mathcal{Q}$ that does not have a quasi- $L R F$ must be infinite.

To overcome this finiteness problem, we use notions similar to the witness and h-witness that we have used for the case of $\operatorname{LinRF}(\mathbb{Z})$. In particular, we show that the existence of $T$ as in Corollary 5.16 can be witnessed by finite sets $X \subseteq I\left(\mathcal{Q}_{1}\right) \cup \cdots \cup I\left(\mathcal{Q}_{k}\right)$ and $Y \subseteq I\left(\mathcal{R}_{\mathcal{Q}_{1}}\right) \cup \cdots \cup I\left(\mathcal{R}_{\mathcal{Q}_{k}}\right)$, whose bit-size is bounded polynomially in the bit-size of the input.

Definition 5.18. Let $X=X_{1} \cup \cdots \cup X_{k}$ and $Y=Y_{1} \cup \cdots \cup Y_{k}$, such that $(a) X_{i} \subseteq I\left(\mathcal{Q}_{i}\right) ;(b) Y_{i} \subseteq$ $I\left(\mathcal{R}_{\mathcal{Q}_{i}}\right)$; and $(c) Y_{i} \neq \emptyset \Rightarrow X_{i} \neq \emptyset$. We say that $X$ and $Y$ form a witness against the existence of 
a $L L R F$ for $I\left(\mathcal{Q}_{1}\right), \ldots, I\left(\mathcal{Q}_{k}\right)$, if the following set of linear constraints, denoted by $\Phi(X, Y)$, has no solution

$$
\begin{aligned}
& \vec{\lambda} \cdot \mathbf{x}+\lambda_{0} \geq 0 \text { for all } \mathbf{x}^{\prime \prime} \in X \\
& \vec{\lambda} \cdot \mathbf{y} \geq 0 \text { for all } \mathbf{y}^{\prime \prime} \in Y \\
& \vec{\lambda} \cdot\left(\mathbf{x}-\mathbf{x}^{\prime}\right) \geq 0 \text { for all } \mathbf{x}^{\prime \prime} \in X \\
& \vec{\lambda} \cdot\left(\mathbf{y}-\mathbf{y}^{\prime}\right) \geq 0 \text { for all } \mathbf{y}^{\prime \prime} \in Y \\
& \sum_{\mathbf{x}^{\prime \prime} \in X} \vec{\lambda} \cdot\left(\mathbf{x}-\mathbf{x}^{\prime}\right)+\sum_{\mathbf{y}^{\prime \prime} \in Y} \vec{\lambda} \cdot\left(\mathbf{y}-\mathbf{y}^{\prime}\right) \geq 1
\end{aligned}
$$

LEMMA 5.19. Let $X=X_{1} \cup \cdots \cup X_{k}$ and $Y=Y_{1} \cup \cdots \cup Y_{k}$ be as in Definition 5.18. Then there is $T \subseteq I\left(\mathcal{Q}_{1}\right) \cup \cdots \cup I\left(\mathcal{Q}_{k}\right)$ that has no non-trivial quasi-LRF.

Proof. We construct such $T$. First note that for $\mathbf{x}^{\prime \prime} \in X_{i}$ and $\mathbf{y}^{\prime \prime} \in Y_{i}$, the point $\mathbf{x}^{\prime \prime}+a \mathbf{y}^{\prime \prime}$, for any integer $a \geq 0$, is a transition in $I\left(\mathcal{Q}_{i}\right)$. Now define

$$
T=\left\{\mathbf{x}^{\prime \prime}+a \mathbf{y}^{\prime \prime} \mid \mathbf{x}^{\prime \prime} \in X_{i}, \mathbf{y}^{\prime \prime} \in Y_{i}, \text { integer } a \geq 0\right\} .
$$

Clearly $T \subseteq I\left(\mathcal{Q}_{1}\right) \cup \cdots \cup I\left(\mathcal{Q}_{k}\right)$. We claim that $T$ has no non-trivial quasi- $L R F$. Assume the contrary, i.e., there is $\left(\lambda_{0}, \vec{\lambda}\right) \in \mathbb{Q}^{n+1}$ such that $\rho(\mathbf{x})=\vec{\lambda} \cdot \mathbf{x}+\lambda_{0}$ is a non-trivial quasi-LRF for $T$. We show that $\left(c \lambda_{0}, c \vec{\lambda}\right)$, for some $c>0$, is a solution of $\Phi(X, Y)$, which contradicts the assumption that $X$ and $Y$ form a witness as in Definition 5.18 .

We first show that $33 \mathrm{a} 33 \mathrm{~d})$ of $\Phi(X, Y)$ hold for $\left(c \lambda_{0}, c \vec{\lambda}\right)$ with any $c>0$. Pick arbitrary $\mathbf{x}^{\prime \prime} \in X_{i}$ and $\mathbf{y}^{\prime \prime} \in Y_{i}$. Since $\rho$ is a non-trivial quasi- LRF for T, inequalities (30]31) on Page 25 must hold for $\mathbf{x}^{\prime \prime}+a \mathbf{y}^{\prime \prime}=\left(\begin{array}{l}\mathbf{x}+a \mathbf{y} \\ \mathbf{x}^{\prime}+a \mathbf{y}^{\prime}\end{array}\right) \in T$. Namely, the following must hold for any integer $a \geq 0$

$$
\begin{aligned}
\rho(\mathbf{x}+a \mathbf{y}) & =\vec{\lambda} \cdot(\mathbf{x}+a \mathbf{y})+\lambda_{0}=\vec{\lambda} \cdot \mathbf{x}+\lambda_{0}+a \vec{\lambda} \cdot \mathbf{y} \geq 0 \\
\Delta \rho\left(\mathbf{x}^{\prime \prime}+a \mathbf{y}^{\prime \prime}\right) & =\vec{\lambda} \cdot(\mathbf{x}+a \mathbf{y})-\vec{\lambda} \cdot\left(\mathbf{x}^{\prime}+a \mathbf{y}^{\prime}\right)=\vec{\lambda} \cdot\left(\mathbf{x}-\mathbf{x}^{\prime}\right)+a \vec{\lambda} \cdot\left(\mathbf{y}-\mathbf{y}^{\prime}\right) \geq 0
\end{aligned}
$$

This implies

(i) $\vec{\lambda} \cdot \mathbf{x}+\lambda_{0} \geq 0$, otherwise (34) is false for $a=0$;

(ii) $\vec{\lambda} \cdot \mathbf{y} \geq 0$, otherwise 34 is false for $a>-\left(\vec{\lambda} \cdot \mathbf{x}+\lambda_{0}\right) /(\vec{\lambda} \cdot \mathbf{y})$;

(iii) $\vec{\lambda} \cdot\left(\mathbf{x}-\mathbf{x}^{\prime}\right) \geq 0$, otherwise $(35)$ is false for $a=0$; and

(iv) $\vec{\lambda} \cdot\left(\mathbf{y}-\mathbf{y}^{\prime}\right) \geq 0$, otherwise 35 is false for $a>-\vec{\lambda} \cdot\left(\mathbf{x}-\mathbf{x}^{\prime}\right) / \vec{\lambda} \cdot\left(\mathbf{y}-\mathbf{y}^{\prime}\right)$.

Note that the inequalities in i iv above are those used in 33a 33d. Hence 33a 33d hold for $\left(\lambda_{0}, \vec{\lambda}\right)$, and clearly, also for $\left(c \lambda_{0}, c \vec{\lambda}\right)$ with any $c>0$.

Now we show that $(33 \mathrm{e})$ of $\Phi(X, Y)$ holds for $\left(c \lambda_{0}, c \vec{\lambda}\right)$, for some $c>0$. Since $\rho$ is a non-trivial quasi- $L R F$, then, inequality 31] must be strict for at least one $\mathbf{x}^{\prime \prime}+a \mathbf{y}^{\prime \prime}=\left(\begin{array}{c}\mathbf{x}+a \mathbf{y} \\ \mathbf{x}^{\prime}+a \mathbf{y}^{\prime}\end{array}\right) \in T$, i.e., $\Delta \rho\left(\mathbf{x}^{\prime \prime}+a \mathbf{y}^{\prime \prime}\right)=\vec{\lambda} \cdot\left(\mathbf{x}-\mathbf{x}^{\prime}\right)+a \vec{\lambda} \cdot\left(\mathbf{y}-\mathbf{y}^{\prime}\right)>0$. This means that either $\vec{\lambda} \cdot\left(\mathbf{x}-\mathbf{x}^{\prime}\right)>0$ or $\vec{\lambda} \cdot\left(\mathbf{y}-\mathbf{y}^{\prime}\right)>0$ must hold. Taking $c>0$ large enough, we have $c \vec{\lambda} \cdot\left(\mathbf{x}-\mathbf{x}^{\prime}\right) \geq 1$ or $c \vec{\lambda} \cdot\left(\mathbf{y}-\mathbf{y}^{\prime}\right) \geq 1$. Thus, inequality (33e) holds for $\left(c \lambda_{0}, c \vec{\lambda}\right)$. Since 33 a $33 \mathrm{~d}$ ) also hold for this $\left(c \lambda_{0}, c \vec{\lambda}\right)$, it is a solution of $\Phi(X, Y)$. 
LEMMA 5.20. If there is $T \subseteq I\left(\mathcal{Q}_{1}\right) \cup \cdots \cup I\left(\mathcal{Q}_{k}\right)$ that has no non-trivial quasi-LRF, then there are finite sets $X=X_{1} \cup \cdots \cup X_{k}$ and $Y=Y_{1} \cup \cdots \cup Y_{k}$, fulfilling the conditions of Definition 5.18.

Proof. Let $\mathcal{Q} \in\left\{\mathcal{Q}_{1}, \ldots, \mathcal{Q}_{k}\right\}$ and consider the generator representations

$$
\mathcal{Q}_{I}=\operatorname{convhull}\left\{\mathbf{x}_{1}^{\prime \prime}, \ldots, \mathbf{x}_{m}^{\prime \prime}\right\}+\operatorname{cone}\left\{\mathbf{y}_{1}^{\prime \prime}, \ldots, \mathbf{y}_{t}^{\prime \prime}\right\} .
$$

Using these representations, every $\mathbf{x}^{\prime \prime} \in T \cap \mathcal{Q}$ can be written as $\mathbf{x}^{\prime \prime}=\sum_{i=1}^{m} a_{i} \mathbf{x}_{i}^{\prime \prime}+\sum_{j=1}^{t} b_{j} \mathbf{y}_{j}^{\prime \prime}$ for some rationals $a_{i}, b_{j} \geq 0$, and $\sum_{i} a_{i}=1$. We let $\operatorname{ver}\left(\mathbf{x}^{\prime \prime}\right)$ be the set of all vertices $\mathbf{x}_{i}^{\prime \prime}$ with $a_{i}>0$ and $\operatorname{rays}\left(\mathbf{x}^{\prime \prime}\right)$ be the set of all rays $\mathbf{y}_{j}^{\prime \prime}$ with $b_{j}>0$. We define $X_{\ell}=\cup\left\{\operatorname{ver}\left(\mathbf{x}^{\prime \prime}\right) \mid \mathbf{x}^{\prime \prime} \in T \cap I\left(\mathcal{Q}_{\ell}\right)\right\}$ and $Y_{\ell}=\cup\left\{\operatorname{rays}\left(\mathbf{x}^{\prime \prime}\right) \mid \mathbf{x}^{\prime \prime} \in T \cap I\left(\mathcal{Q}_{\ell}\right)\right\}$; for $1 \leq \ell \leq k$. Next we show that $X=X_{1} \cup \cdots \cup X_{k}$ and $Y=Y_{1} \cup \cdots \cup Y_{k}$ form a witness as in Definition 5.18 .

Conditions (a b) of Definition 5.18 hold by construction, and Condition (c) holds because $\sum_{i} a_{i}=1$. What is left to show is that $\Phi(X, Y)$ has no solution. Assume the contrary, i.e., $\Phi(X, Y)$ has a solution $\left(\lambda_{0}, \vec{\lambda}\right) \in \mathbb{Q}^{n+1}$. We claim that then $\rho(\mathbf{x})=\vec{\lambda} \cdot \mathbf{x}+\lambda_{0}$ is a non-trivial quasi- $L R F$ for $T$, which contradicts the lemma's assumption. Pick an arbitrary $\mathbf{x}^{\prime \prime} \in T$ and write it, using the corresponding $X_{\ell}$ and $Y_{\ell}$, as $\mathbf{x}^{\prime \prime}=\sum_{i=1}^{m} a_{i} \mathbf{x}_{i}^{\prime \prime}+\sum_{j=1}^{t} b_{j} \mathbf{y}_{j}^{\prime \prime}$ where $a_{i}, b_{j}>0$ and $\sum_{i=1}^{m} a_{i}=1$. Since $33 \mathrm{a} 33 \mathrm{c}$ hold for each $\mathbf{x}_{i}^{\prime \prime} \in X$ and $33 \mathrm{~b} 33 \mathrm{~d}$ ) hold for each $\mathbf{y}_{j}^{\prime \prime} \in Y$, we have

$$
\begin{aligned}
\rho(\mathbf{x}) & =\vec{\lambda} \cdot\left(\sum_{i=1}^{m} a_{i} \mathbf{x}_{i}+\sum_{j=1}^{t} b_{j} \mathbf{y}_{j}\right)+\lambda_{0} \\
& =\sum_{i=1}^{m} a_{i} \cdot\left(\vec{\lambda} \cdot \mathbf{x}_{i}+\lambda_{0}\right)+\sum_{j=1}^{t} b_{j} \vec{\lambda} \cdot \mathbf{y}_{j} \geq 0 \\
\Delta \rho\left(\mathbf{x}^{\prime \prime}\right) & =\vec{\lambda} \cdot\left(\sum_{i=1}^{m} a_{i} \mathbf{x}_{i}+\sum_{j=1}^{t} b_{j} \mathbf{y}_{j}\right)-\vec{\lambda} \cdot\left(\sum_{i=1}^{m} a_{i} \mathbf{x}_{i}^{\prime}+\sum_{j=1}^{t} b_{j} \mathbf{y}_{j}^{\prime}\right) \\
& =\sum_{i=1}^{m} a_{i} \vec{\lambda} \cdot\left(\mathbf{x}_{i}-\mathbf{x}_{i}^{\prime}\right)+\sum_{j=1}^{t} b_{j} \vec{\lambda} \cdot\left(\mathbf{y}_{j}-\mathbf{y}_{j}^{\prime}\right) \geq 0
\end{aligned}
$$

Thus, $\rho$ satisfies 30 31] for any $\mathbf{x}^{\prime \prime} \in T$. Now since (33e) holds, there must be $\mathbf{x}_{i}^{\prime \prime}$ or $\mathbf{y}_{j}^{\prime \prime}$ for which $\vec{\lambda} \cdot\left(\mathbf{x}_{i}-\mathbf{x}_{i}^{\prime}\right)>0$ or $\vec{\lambda} \cdot\left(\mathbf{y}_{j}-\mathbf{y}_{j}^{\prime}\right)>0$. Take $\mathbf{x}^{\prime \prime} \in T$ from which these $\mathbf{x}_{i}^{\prime \prime}$ or $\mathbf{y}_{i}^{\prime \prime}$ come, then it must be the case that $\Delta \rho\left(\mathbf{x}^{\prime \prime}\right)>0$, i.e., inequality (31) is strict for $\mathbf{x}^{\prime \prime}$.

EXAMPLE 5.21. For $\mathcal{Q}=\left\{x^{\prime} \leq x-1\right\}$ of Example 5.17, we claim that $X=\{(0,-1)\}$ and $Y=\{(1,1),(-1,-1)\}$ form a witness as in Definition 5.18. It is easy to check that $X$ and $Y$ satisfy conditions (a). Then, $\Phi(X, Y)$ is the set of inequalities $\left\{\lambda_{0} \geq 0, \lambda_{1} \geq 0,-\lambda_{1} \geq 0, \lambda_{1} \geq 1\right\}$ which has no solution.

EXAMPLE 5.22. Consider an $M L C$ loop represented by

$$
\begin{aligned}
& \mathcal{Q}_{1}=\left\{x_{1} \geq 0, \quad x_{2} \geq 0, \quad x_{1}^{\prime}=x_{1}-1\right\} \\
& \mathcal{Q}_{2}=\left\{x_{1} \geq 0, \quad x_{2} \geq 0, \quad x_{2}^{\prime}=x_{2}-1\right\}
\end{aligned}
$$

and let

$$
\begin{aligned}
& X_{1}=\{(0,0,-1,0)\}, \quad Y_{1}=\{(0,0,0,1)\} \\
& X_{2}=\{(0,0,0,-1)\}, \quad Y_{2}=\{(0,0,1,0)\}
\end{aligned}
$$


We claim that these sets form a witness as in Definition 5.18. It is easy to check that they satisfy conditions (a c) of Definition 5.18 Substituting these points in (33e) gives $0 \geq 1$, so clearly (33a 33e) are unsatisfiable.

The next lemma concerns the bit-size of the witness.

LEMMA 5.23. If there is a finite witness for the nonexistence of $L L R F$ for $I\left(\mathcal{Q}_{1}\right), \ldots, I\left(\mathcal{Q}_{k}\right)$, then there is one defined by $X=X_{1} \cup \cdots \cup X_{k}$ and $Y=Y_{1} \cup \cdots \cup Y_{k}$ such that $\sum_{i=1}^{k}\left|X_{i}\right|+\left|Y_{i}\right| \leq 6 n+2$; and its bit-size is polynomial in the bit-size of $\mathcal{Q}_{1}, \ldots, \mathcal{Q}_{k}$.

Proof. Consider the witness constructed in Lemma 5.20, and recall that $\Phi_{1}=\Phi(X, Y)$ has no solution. Let $Z$ be any maximal linearly-independent subset of $X \cup Y$. Clearly, $|Z| \leq 2 n$. Let $\Phi_{2}$ be the formula obtained from $\Phi_{1}$ by replacing (33e) with

$$
\sum_{\mathbf{z}^{\prime \prime} \in Z} \vec{\lambda} \cdot\left(\mathbf{z}-\mathbf{z}^{\prime}\right) \geq 1
$$

We claim that $\Phi_{2}$ has no solution. To see this, take arbitrary $\left(\lambda_{0}, \vec{\lambda}\right) \in \mathbb{Q}^{2 n}$, we know it is not a solution of $\Phi_{1}$. If this is because one of the inequalities in (33a) 33d is false, then it is clearly not a solution of $\Phi_{2}$ since it includes all such inequalities. If all inequalities in (33a $33 \mathrm{~d}$ ) are true, then (33e) must be false. Since all terms in the sum are non-negative, they must all be zero, that is, $\vec{\lambda} \cdot\left(\mathbf{z}-\mathbf{z}^{\prime}\right)=0$ for any $\mathbf{z}^{\prime \prime} \in X \cup Y$. Otherwise, $\left(c \lambda_{0}, \overrightarrow{c \lambda}\right)$ for $c \geq 1$ large enough would be a solution of $\Phi_{1}$. Thus, inequality (36) is false.

A corollary of Farkas' Lemma Schrijver 1986, p. 94] states that: if a set of inequalities over $\mathbb{Q}^{d}$ has no solution, there is a subset of at most $d+1$ inequalities that has no solution. Let $\Phi_{3}$ be such a subset of $\Phi_{2}$, it has at most $n+2$ inequalities (since $\Phi_{2}$ is over $\mathbb{Q}^{n+1}$ ). Note that $\Phi_{3}$ must include inequality (36), otherwise it is trivially satisfiable. Let $X^{\prime}=X_{1}^{\prime} \cup \ldots \cup X_{k}^{\prime} \subseteq X$ and $Y^{\prime}=Y_{1}^{\prime} \cup \ldots \cup Y_{k}^{\prime} \subseteq Y$ be the points involved in the inequalities of $\Phi_{3}$ (including (36)), then $\sum_{i=1}^{k}\left|X_{i}^{\prime}\right|+\left|Y_{i}^{\prime}\right| \leq n+1+2 n=3 n+1$. To get a witness as per Definition 5.18, if, for any $i \leq k, Y_{i}^{\prime} \neq \emptyset$ and $X_{i}^{\prime}=\emptyset$, we include an arbitrary point $\mathbf{x}^{\prime \prime} \in X_{i}$ to $X_{i}^{\prime}$. This can at most double the size of these sets, i.e., $\sum_{i=1}^{k}\left|X_{i}^{\prime}\right|+\left|Y_{i}^{\prime}\right| \leq 6 n+2$ (or $\sum_{i=1}^{k}\left|X_{i}^{\prime}\right|+\left|Y_{i}^{\prime}\right| \leq 3 n+1+k$ when $\left.k<3 n+1\right)$.

We claim that $\left\langle X^{\prime}, Y^{\prime}\right\rangle$ is a witness that fulfills the conditions of Definition 5.18. It satisfies conditions (a) by construction. Next, we show that $\Phi_{4}=\Phi\left(X^{\prime}, Y^{\prime}\right)$ has no solution. Take arbitrary $\left(\lambda_{0}, \vec{\lambda}\right) \in \mathbb{Q}^{n+1}$, we know it is not a solution for $\Phi_{2}$. If it is because one of the inequalities in 33a 33d is false, then it is clearly not a solution of $\Phi_{4}$ since it includes all such inequalities. If all inequalities in (33a 33d) are true, then (36) must be false, and then we must have $\vec{\lambda} \cdot\left(\mathbf{z}-\mathbf{z}^{\prime}\right)=0$ for any $\mathbf{z}^{\prime \prime} \in Z$. Now since any $\mathbf{z}^{\prime \prime} \in X^{\prime} \cup Y^{\prime}$ is a linear combination of points from $Z, \vec{\lambda} \cdot\left(\mathbf{x}-\mathbf{x}^{\prime}\right)=0$ for any $\mathbf{x}^{\prime \prime} \in X^{\prime}$ and $\vec{\lambda} \cdot\left(\mathbf{y}-\mathbf{y}^{\prime}\right)=0$ for any $\mathbf{y}^{\prime \prime} \in Y^{\prime}$. Thus, inequality $\left[33 \mathrm{e}\right.$ of $\Phi_{4}$ is false.

Finally, we show that the bit-size of the witness is polynomial in the bit-size of the input. Recall that the points of $X^{\prime}$ and $Y^{\prime}$ come from the generator representations of $\mathcal{Q}_{1 I}, \ldots, \mathcal{Q}_{k_{I}}$, and that there is a generator representation for each $\mathcal{Q}_{i I}$ in which each vertex/ray can fit in $\left\|\mathcal{Q}_{i_{I}}\right\|_{v}$ bits. Thus, the bit-size of $X^{\prime}$ and $Y^{\prime}$ is bounded by $(6 n+2) \cdot \max _{i}\left\|\mathcal{Q}_{i I}\right\|_{v}$. By Theorem 2.2 , since the dimension of each $\mathcal{Q}_{i}$ is $2 n$,

$$
(6 n+2) \cdot \max _{i}\left\|\mathcal{Q}_{i I}\right\|_{v} \leq(6 n+2) \cdot\left(6 \cdot(2 n)^{3} \cdot \max _{i}\left\|\mathcal{Q}_{i}\right\|_{f}\right) \leq\left(288 n^{4}+96 n^{3}\right) \cdot \max _{i}\left\|\mathcal{Q}_{i}\right\|_{b}
$$

which is polynomial in the bit-size of the input. 
THEOREM 5.24. LEXLinRF $(\mathbb{Z}) \in$ coNP for $M L C$ loops.

Proof. We show that the complement of $\operatorname{LinRF}(\mathbb{Z})$ has a polynomially checkable witness. The witness is a listing of sets of integer points $X=X_{1} \cup \cdots \cup X_{k}$ and $Y=Y_{1} \cup \cdots \cup Y_{k}$ of at most $6 n+2$ elements and has a polynomial bit-size (specifically, a bit-size bounded as in Lemma 5.23). Verifying a witness consists of the following steps:

Step 1. Verify that each $\mathbf{x}^{\prime \prime} \in X_{i}$ is in $I\left(\mathcal{Q}_{i}\right)$, which can be done by verifying $A_{i}^{\prime \prime} \mathbf{x}^{\prime \prime} \leq \mathbf{c}_{i}^{\prime \prime}$; and that each $\mathbf{y}^{\prime \prime} \in Y_{i}$ is in $I\left(\mathcal{R}_{\mathcal{Q}}\right)$, which can be done by verifying $A_{i}^{\prime \prime} \mathbf{y}^{\prime \prime} \leq 0$. This is done in polynomial time. Note that according to Lemma 5.19 it is not necessary to check that $X$ and $Y$ come from a particular generator representation.

Step 2. Verify that $\Phi(X, Y)$ has no solutions, which can be done in polynomial time since it is an $L P$ problem over $\mathbb{Q}^{n+1}$.

\subsection{Lexicographic ranking functions over the rationals}

In this section we address the $\operatorname{LiNRF}(\mathbb{Q})$ problem. In particular, we show that Procedure LLRFSYN, when applied to the input polyhedra $\mathcal{Q}_{1}, \ldots, \mathcal{Q}_{k}$ instead of their integer hulls, can be used to decide the existence of a $L L R F$ for $\mathcal{Q}_{1}, \ldots, \mathcal{Q}_{k}$. However, in such case, the returned value $\tau=\left\langle\rho_{1}, \ldots, \rho_{d}\right\rangle$ of the algorithm does not fit in the class of $L L R F s$ as in Definition 2.5. We define a new class of $L L R F s$ that captures such functions, and prove that it is actually equivalent to that of Definition 2.5 as far as the existence of a $L L R F$ is concerned.

First recall that in Section 2.4 we discussed the possibility of replacing inequality $\Delta \rho_{i}\left(\mathbf{x}^{\prime \prime}\right) \geq 1$ by $\Delta \rho_{i}\left(\mathbf{x}^{\prime \prime}\right) \geq \delta_{i}$ in condition 10 of Definition 2.5. With this change, $\tau=\left\langle\rho_{1}, \ldots, \rho_{d}\right\rangle$ is a LLRF if and only if there are positive $\delta_{1}, \ldots, \delta_{d}$ such that, for any $\mathbf{x}^{\prime \prime} \in \mathcal{Q}_{1} \cup \cdots \cup \mathcal{Q}_{k}$ there exists $i$ for which the following hold

$$
\begin{aligned}
\forall j<i . \Delta \rho_{j}\left(\mathbf{x}^{\prime \prime}\right) & \geq 0 \\
\forall j \leq i . \quad \rho_{j}(\mathbf{x}) & \geq 0 \\
\Delta \rho_{i}\left(\mathbf{x}^{\prime \prime}\right) & \geq \delta_{i}
\end{aligned}
$$

This is equivalent to Definition 2.5, as far as the existence of a $L L R F$ is concerned, since $c \tau$, for any $c>\min \left(\delta_{i}\right)^{-1}$, is a corresponding $L L R F$ as in Definition 2.5. In the rest of this section, for the sake of simplifying the formal presentation, we use this notion of LLRFs.

Let us start by explaining why the returned value of Procedure LLRFSYN, in the rational case, does not fit in the above class of $L L R F s$. For this, let us consider a non-trivial quasi- $L R F \rho$ synthesized at Line 2 In the integer case, all integer transitions of $\mathcal{P}_{1}, \ldots, \mathcal{P}_{k}$ that do not pass to $\mathcal{P}_{1}^{\prime}, \ldots, \mathcal{P}_{k}^{\prime}$ are ranked by this $\rho$. This is because $\Delta \rho\left(\mathbf{x}^{\prime \prime}\right) \geq 1$ for all such transitions (see the proof of Lemma 5.9, point (3p). This, however, is not true when considering rational transitions. In this case, all transitions that do not pass to $\mathcal{P}_{1}^{\prime}, \ldots, \mathcal{P}_{k}^{\prime}$ satisfy $\Delta \rho\left(\mathbf{x}^{\prime \prime}\right)>0$, but it is not guaranteed that $\Delta \rho\left(\mathbf{x}^{\prime \prime}\right)$ has a minimum $\delta$ over this set of transitions. For example, take $\mathcal{P}_{1}=\left\{x \geq 0, x=2 x^{\prime}\right\}$ and $\rho(x)=x$, then $\mathcal{P}_{1}^{\prime}=\left\{x=0, x^{\prime}=0\right\}$. The transitions that do not pass to $\mathcal{P}_{1}^{\prime}$ are those specified by the non-closed polyhedron $\left\{x>0, x=2 x^{\prime}\right\}$, in which $\Delta \rho$ does not have a positive lower bound. This leads us to introduce weak LLRFs. 
Definition 5.25. We say that $\tau=\left\langle\rho_{1}, \ldots, \rho_{d}\right\rangle$ is a weak $L L R F$ for $\mathcal{Q}_{1} \cup \cdots \cup \mathcal{Q}_{k}$, if and only if for any $\mathbf{x}^{\prime \prime} \in \mathcal{Q}_{1} \cup \cdots \cup \mathcal{Q}_{k}$ there exists $i$ for which $(3738)$ hold, as well as

$$
\Delta \rho_{i}\left(\mathbf{x}^{\prime \prime}\right)>0
$$

(which replaces $(39)$ ).

While any $L L R F$ is a also weak $L L R F$, the converse is more subtle. Over the integers, the existence of a weak $L L R F$ implies the existence of a $L L R F$ (see Lemma 5.6). Over the rationals, this is not immediate, and whether such a function implies termination is, at least, unclear, as infinitely descending sequences of positive rationals exist.

EXAMPLE 5.26. Consider the following $M L C$ loop

$$
\begin{array}{rll}
\text { loop : } & \left\{x_{1} \geq 0,\right. & \left.x_{1}^{\prime}=x_{1}-1\right\} \\
& \vee\left\{x_{1} \geq 0, \quad x_{2}-x_{1} \geq 0,\right. & \left.x_{1}^{\prime}=x_{1}, \quad x_{2}^{\prime}=x_{2}-1\right\} \\
& \vee\left\{x_{1} \geq 0, \quad x_{2}-x_{1} \geq 0, \quad x_{3} \geq 0, \quad x_{1}^{\prime} \leq \frac{1}{2} x_{1}, \quad x_{2}^{\prime}=x_{2}, \quad x_{3}^{\prime}=x_{3}-1\right\}
\end{array}
$$

Applying Procedure LLRFSYN to the corresponding transition polyhedra $\mathcal{Q}_{1}, \mathcal{Q}_{2}, \mathcal{Q}_{3}$ possibly returns $\tau=\left\langle x_{1}, x_{2}-x_{1}, x_{3}\right\rangle$. It is easy to see that it is a weak $L L R F$ over the rationals, and, consequently, it is a $L L R F$ over the integers. To see why it is not a $L L R F$ over the rationals, assume the first component of $\tau$ decreases by at least $\delta_{1}>0$. All transitions for which $x_{1}-x_{1}^{\prime}<\delta_{1}$ are not ranked by this component and thus should be ranked by either the second or the third. Let us take $\mathbf{x}^{\prime \prime} \in \mathcal{Q}_{3}$ such that $\mathbf{x}=\left(\delta_{1}, 1,1\right)$ and $\mathbf{x}^{\prime}=\left(\frac{1}{2} \delta_{1}, 1,0\right)$. This transition is not ranked by the first component since $\Delta \rho_{1}\left(\mathbf{x}^{\prime \prime}\right)=\frac{1}{2} \delta_{1}<\delta_{1}$, and it is not ranked by the second or the third since $\Delta \rho_{2}\left(\mathbf{x}^{\prime \prime}\right)=-\frac{1}{2} \delta_{1}<0$. Nonetheless, this loop is terminating over the rationals and has a $L L R F$, and later we show how to obtain it.

Over the rationals, Procedure LLRFSYN is sound and complete for synthesizing weak LLRFs. Moreover, as in the integer case, it synthesizes one with minimal dimension.

LEMMA 5.27. If $\operatorname{LLRFSYN}\left(\left\langle\mathcal{Q}_{1}, \ldots, \mathcal{Q}_{k}\right\rangle\right)$ returns $\tau$ different from NoNE, then $\tau$ is a weak LLRF for $\mathcal{Q}_{1}, \ldots, \mathcal{Q}_{k}$.

Proof. The proof is as that of Lemma 5.9. The differences are: we drop point (1) regards the integrality of corresponding polyhedra; and in point (3) we use $\Delta \rho\left(\mathbf{x}^{\prime \prime}\right)>0$ instead of $\Delta \rho\left(\mathbf{x}^{\prime \prime}\right) \geq 1$.

LEMMA 5.28. Procedure LLRFSYN, when applied to $\mathcal{Q}_{1}, \cdots, \mathcal{Q}_{k}$, is sound and complete for the existence of a weak LLRF for $\mathcal{Q}_{1}, \ldots, \mathcal{Q}_{k}$. Moreover, when it finds a weak LLRF, it finds one of a minimal dimension.

Proof. The proof is as that of Theorem 5.12. First note that the sufficient and necessary condition for the existence of a $L L R F$, stated in Observations 5.10 and 5.11 is a condition for existence of a weak $L L R F$ when applied to $\mathcal{Q}_{1}, \ldots, \mathcal{Q}_{k}$. Then, together with Lemma 5.27 we get soundness and completeness. The minimality follows from the same consideration in the proof of Theorem 5.12

In the rest of this section we show how one can construct a $L L R F$ for $\mathcal{Q}_{1}, \ldots, \mathcal{Q}_{k}$ from a weak $L L R F$. This implies soundness and completeness of Procedure LLRFSYN as a decision procedure for $\operatorname{LEx} \operatorname{Lin} R F(\mathbb{Q})$, and its usage for synthesis of LLRFs. To simplify notation, we shall consider the polyhedra $\mathcal{Q}_{1}, \ldots, \mathcal{Q}_{k}$ to be fixed up to the completion of the proof. 
Definition 5.29. Let $\tau=\left\langle\rho_{1}, \ldots, \rho_{d}\right\rangle$ be a weak $L L R F$ for $\mathcal{Q}_{1}, \ldots, \mathcal{Q}_{k}$. The ranking chain for $\tau$ is the $(d+1)$-tuple of sets, $U_{1}, \ldots, U_{d+1}$, defined by $U_{1}=\mathcal{Q}_{1} \cup \cdots \cup \mathcal{Q}_{k}$, and $U_{i+1}=U_{i} \wedge\left(\Delta \rho_{i}\left(\mathbf{x}^{\prime \prime}\right)=0\right)$.

Observe that

$$
\mathcal{Q}_{1} \cup \cdots \cup \mathcal{Q}_{k}=U_{1} \supseteq U_{2} \supseteq \cdots \supseteq U_{d} \supseteq U_{d+1}=\emptyset .
$$

It is easy to see that if for some $j, U_{j}=U_{j+1}$, it is possible to omit $\rho_{j}$ from $\tau$ without any harm. We say that $\tau$ is irredundant if

$$
\mathcal{Q}_{1} \cup \cdots \cup \mathcal{Q}_{k}=U_{1} \supset U_{2} \supset \cdots \supset U_{d} \supset U_{d+1}=\emptyset .
$$

Observation 5.30. A weak $L L R F$ computed by Procedure LLRFSYN is irredundant. In fact, $U_{i}$ is the union $\mathcal{P}_{1} \cup \cdots \cup \mathcal{P}_{k}$ of the arguments to the $i$-th recursive call.

By the definition of a weak $L L R F$, and the definition of $U_{1}, \ldots, U_{d+1}$, the following properties clearly follow:

$$
\begin{aligned}
\forall \mathbf{x}^{\prime \prime} \in U_{i} . \quad \rho_{i}(\mathbf{x}) & \geq 0, \\
\forall \mathbf{x}^{\prime \prime} \in U_{i} \backslash U_{i+1} \cdot \Delta \rho_{i}\left(\mathbf{x}^{\prime \prime}\right) & >0, \\
\forall \mathbf{x}^{\prime \prime} \in U_{i+1} \cdot \Delta \rho_{i}\left(\mathbf{x}^{\prime \prime}\right) & =0 .
\end{aligned}
$$

Note that each $U_{i}$ is a finite union of closed polyhedra, obtained by intersecting $U_{1}$ with some hyperplanes. For $1 \leq i \leq d$, let $J_{i}=\left\{j \mid \mathcal{Q}_{j} \cap U_{i} \neq \emptyset\right\}$, and let $\bar{U}_{i}=\bigcup_{j \in J_{i}} \mathcal{Q}_{j}$. This means that if $U_{i}$ includes a point from $\mathcal{Q}_{j}$, then $\bar{U}_{i}$ includes all points of $\mathcal{Q}_{j}$. Note that $\bar{U}_{i} \supseteq \bar{U}_{i+1}$. The next lemma shows that one can construct, for each $U_{i}$, a function $f_{i}$ such that the domain on which $\left(44_{i}\right)$ holds is extended to $\bar{U}_{i} \backslash U_{i+1}$. These functions are later used in constructing a $L L R F$ for $\mathcal{Q}_{1}, \ldots, \mathcal{Q}_{k}$.

LEMMA 5.31. Given an irredundant weak $L L R F, \tau$, and its ranking chain $\left\{U_{i}\right\}$, one can construct, for each $1 \leq i \leq d$, an affine function $f_{i}: \mathbb{Q}^{n} \rightarrow \mathbb{Q}$ such that

$$
\begin{aligned}
& \forall \mathbf{x}^{\prime \prime} \in U_{i} \cdot f_{i}(\mathbf{x}) \geq \rho_{i}(\mathbf{x}) \geq 0 \\
& \forall \mathbf{x}^{\prime \prime} \in \bar{U}_{i} \backslash U_{i+1} . \quad \Delta f_{i}\left(\mathbf{x}^{\prime \prime}\right)>0 \\
& \forall \mathbf{x}^{\prime \prime} \in U_{i+1} . \quad \Delta f_{i}\left(\mathbf{x}^{\prime \prime}\right)=0 .
\end{aligned}
$$

Proof. The proof proceeds by induction.

Base-case. For the base-case we take $i=1$, and define $f_{1}(\mathbf{x})=\rho_{1}(\mathbf{x})$. Since $\bar{U}_{1}=U_{1}, 46_{1}, 48_{1}$ hold (they are equivalent to $43_{1}, 45_{1}$ in this case).

Induction hypothesis. Let $1 \leq i<d$, and assume that $f_{1}, \ldots, f_{i}$ have been defined. In particular, $f_{i}$ satisfies $46_{i} 48_{i}$. Only $f_{i}$ is used in the induction step below. 
Induction step. We show that $f_{i+1}(\mathbf{x})=\rho_{i+1}(\mathbf{x})+(\xi+1) \cdot f_{i}(\mathbf{x})$, for some $\xi \geq 0$, satisfies $46_{i+1} 48_{i+1}$. Most of the proof deals with finding $\xi$ and constructing some related properties. Consider $\mathbf{x}^{\prime \prime} \in U_{i+1}$. If $\mathbf{x}^{\prime \prime} \in U_{i+1}$ then by $\left(48_{i}\right)$ we have $\Delta f_{i}\left(\mathbf{x}^{\prime \prime}\right)=0$, and if $\mathbf{x}^{\prime \prime} \notin U_{i+1}$ then $\mathbf{x}^{\prime \prime} \in \bar{U}_{i+1} \backslash U_{i+1} \subseteq \bar{U}_{i} \backslash U_{i+1}$ and by $\left.\sqrt\left[47_{i}\right)\right]{ }$ we have $\Delta f_{i}\left(\mathbf{x}^{\prime \prime}\right)>0$. This means that the conjunction $\mathbf{x}^{\prime \prime} \in \bar{U}_{i+1} \wedge \Delta f_{i}\left(\mathbf{x}^{\prime \prime}\right) \leq 0$ refers only to the points of $U_{i+1}$, and such points, by $\left.444_{i+1} \mid 45_{i+1}\right)$, satisfy $\Delta \rho_{i+1}\left(\mathbf{x}^{\prime \prime}\right) \geq 0$. Thus, we get

$$
\mathbf{x}^{\prime \prime} \in \bar{U}_{i+1} \wedge \Delta f_{i}\left(\mathbf{x}^{\prime \prime}\right) \leq 0 \Rightarrow \Delta \rho_{i+1}\left(\mathbf{x}^{\prime \prime}\right) \geq 0 .
$$

Take $j \in J_{i+1}$, since $\mathcal{Q}_{j} \subseteq \bar{U}_{i+1}, 49$ still holds when replacing $\bar{U}_{i+1}$ by $\mathcal{Q}_{j}$

$$
\mathbf{x}^{\prime \prime} \in \mathcal{Q}_{j} \wedge \Delta f_{i}\left(\mathbf{x}^{\prime \prime}\right) \leq 0 \Rightarrow \Delta \rho_{i+1}\left(\mathbf{x}^{\prime \prime}\right) \geq 0 .
$$

Note that $(50)$ has a non-vacant antecedent since $U_{i+1} \cap \mathcal{Q}_{j} \neq \emptyset$ by definition of $J_{i+1}$, this allows using Farkas' lemma below. Let $\rho_{i+1}(\mathbf{x})=\vec{a} \cdot \mathbf{x}+a_{0}$ and $f_{i}(\mathbf{x})=\vec{b} \cdot \mathbf{x}+b_{0}$, where $\vec{a}$ and $\vec{b}$ are row vectors of $n$ elements each. Recall that $\mathcal{Q}_{j}$ is given as a system of inequalities $A_{j}^{\prime \prime} \mathbf{x}^{\prime \prime} \leq \mathbf{c}_{j}^{\prime \prime}$, where $A_{j}^{\prime \prime}$ is a matrix of dimension $m \times 2 n$. Using these representations for $\rho_{i+1}, f_{i}$, and $\mathcal{Q}_{j}$ we can present (50) as follows:

$$
\frac{\left(\begin{array}{cc}
A_{j}^{\prime \prime} \\
\vec{b}, & -\vec{b}
\end{array}\right) \cdot \mathbf{x}^{\prime \prime} \leq\left(\begin{array}{c}
\mathbf{c}_{j}^{\prime \prime} \\
0
\end{array}\right)}{\left(\begin{array}{ll}
-\vec{a}, & \vec{a}
\end{array}\right) \cdot \mathbf{x}^{\prime \prime} \leq 0}
$$

Farkas' Lemma guarantees the existence of a vector $\vec{\mu}_{j}=\left(\mu_{j 1}, \ldots, \mu_{j m}\right) \geq 0$, and a scalar $\xi_{j} \geq 0$, such that

$$
\begin{aligned}
-\vec{\mu}_{j} \cdot A_{j}^{\prime \prime}+\xi_{j} \cdot(-\vec{b}, \quad \vec{b}) & =\left(\begin{array}{ll}
\vec{a}, \quad-\vec{a}
\end{array}\right), \\
\vec{\mu}_{j} \cdot \mathbf{c}_{j}^{\prime \prime} & \leq 0 .
\end{aligned}
$$

This means that

$$
\left(\vec{a}+\xi_{j} \cdot \vec{b}, \quad-\left(\vec{a}+\xi_{j} \cdot \vec{b}\right)\right)=-\vec{\mu}_{j} \cdot A_{j}^{\prime \prime}
$$

Now since the entries of $\vec{\mu}_{j}$ are non-negative, from $A_{j}^{\prime \prime} \mathbf{x}^{\prime \prime} \leq \mathbf{c}_{j}^{\prime \prime}$ we get $\vec{\mu}_{j} \cdot A_{j}^{\prime \prime} \mathbf{x}^{\prime \prime} \leq \vec{\mu}_{j} \cdot \mathbf{c}_{j}^{\prime \prime} \leq 0$. By 53 ,

$$
-\vec{\mu}_{j} \cdot A_{j}^{\prime \prime} \mathbf{x}^{\prime \prime}=\left(\vec{a}+\xi_{j} \cdot \vec{b}, \quad-\left(\vec{a}+\xi_{j} \cdot \vec{b}\right)\right) \cdot \mathbf{x}^{\prime \prime}=\left(\vec{a}+\xi_{j} \cdot \vec{b}\right) \cdot\left(\mathbf{x}-\mathbf{x}^{\prime}\right),
$$

so we get

$$
\forall \mathbf{x}^{\prime \prime} \in \mathcal{Q}_{j} \cdot\left(\vec{a}+\xi_{j} \cdot \vec{b}\right) \cdot\left(\mathbf{x}-\mathbf{x}^{\prime}\right) \geq 0 .
$$

Note that $\xi_{j} \cdot \vec{b} \cdot\left(\mathbf{x}-\mathbf{x}^{\prime}\right)=\xi_{j} \cdot \Delta f_{i}\left(\mathbf{x}^{\prime \prime}\right)$, and that by $47_{i} 48_{i}$ we have $\Delta f_{i}\left(\mathbf{x}^{\prime \prime}\right) \geq 0$ over $\bar{U}_{i}$, and thus over $\mathcal{Q}_{j} \subseteq \bar{U}_{i+1} \subseteq \bar{U}_{i}$. This means that (54) still holds when replacing $\xi_{j}$ by any $\xi \geq \xi_{j}$. Now define $\xi=\max \left\{\xi_{j} \mid j \in J_{i+1}\right\}$, then 54 holds for any $j \in J_{i+1}$ and this $\xi$. Since $\bar{U}_{i+1}=\bigcup_{j \in J_{i+1}} \mathcal{Q}_{j}$, we get

$$
\forall \mathbf{x}^{\prime \prime} \in \bar{U}_{i+1} \cdot(\vec{a}+\xi \cdot \vec{b}) \cdot\left(\mathbf{x}-\mathbf{x}^{\prime}\right) \geq 0 .
$$

Now we show that $f_{i+1}(\mathbf{x})=\rho_{i+1}(\mathbf{x})+(\xi+1) \cdot f_{i}(\mathbf{x})$ satisfies $46_{i+1} 48_{i+1}$.

(46-1 By $46_{i}$ ) we know that $f_{i}(\mathbf{x}) \geq 0$ over $U_{i} \supset U_{i+1}$, and by $43_{i}$ ) we know that $\rho_{i+1}(\mathbf{x}) \geq 0$ over $U_{i+1}$. Thus, for any $\mathbf{x}^{\prime \prime} \in U_{i+1}$ we have $f_{i+1}(\mathbf{x})=\rho_{i+1}(\mathbf{x})+(\xi+1) \cdot f_{i}(\mathbf{x}) \geq \rho_{i+1}(\mathbf{x}) \geq 0$. 
447 Pick an arbitrary $\mathbf{x}^{\prime \prime} \in \bar{U}_{i+1} \backslash U_{i+2}$, and consider the two complementary cases $\mathbf{x}^{\prime \prime} \in U_{i+1} \backslash U_{i+2}$ and $\mathbf{x}^{\prime \prime} \notin U_{i+1} \backslash U_{i+2}$ :

(a) If $\mathbf{x}^{\prime \prime} \in U_{i+1} \backslash U_{i+2} \subseteq U_{i+1}$, then by $\left.48_{i}\right)$ we get $\Delta f_{i}\left(\mathbf{x}^{\prime \prime}\right)=0$ and by $44_{i+1}$ we get $\Delta \rho_{i+1}\left(\mathbf{x}^{\prime \prime}\right)>0$. Thus, $\Delta f_{i+1}\left(\mathbf{x}^{\prime \prime}\right)=\Delta \rho_{i+1}\left(\mathbf{x}^{\prime \prime}\right)+(\xi+1) \cdot \Delta f_{i}\left(\mathbf{x}^{\prime \prime}\right)=\Delta \rho_{i+1}\left(\mathbf{x}^{\prime \prime}\right)>0$;

(b) If $\mathbf{x}^{\prime \prime} \notin U_{i+1} \backslash U_{i+2}$, then $\mathbf{x}^{\prime \prime} \in\left(\bar{U}_{i+1} \backslash U_{i+1}\right) \backslash U_{i+2}=\bar{U}_{i+1} \backslash U_{i+1}$. Write $\Delta f_{i+1}\left(\mathbf{x}^{\prime \prime}\right)$ as $(\vec{a}+\xi \cdot \vec{b}) \cdot\left(\mathbf{x}-\mathbf{x}^{\prime}\right)+\Delta f_{i}\left(\mathbf{x}^{\prime \prime}\right)$. On one hand $\mathbf{x}^{\prime \prime} \in \bar{U}_{i+1} \backslash U_{i+1} \subseteq \bar{U}_{i+1}$ so by (55) we get $(\vec{a}+\xi \cdot \vec{b}) \cdot\left(\mathbf{x}-\mathbf{x}^{\prime}\right) \geq 0$, and on the other hand $\mathbf{x}^{\prime \prime} \in \bar{U}_{i+1} \backslash U_{i+1} \subseteq \bar{U}_{i} \backslash U_{i+1}$ so by $\left.47_{i}\right)$ we get $\Delta f_{i}\left(\mathbf{x}^{\prime \prime}\right)>0$. Thus $\Delta f_{i+1}\left(\mathbf{x}^{\prime \prime}\right)=(\vec{a}+\xi \cdot \vec{b}) \cdot\left(\mathbf{x}-\mathbf{x}^{\prime}\right)+\Delta f_{i}\left(\mathbf{x}^{\prime \prime}\right) \geq \Delta f_{i}\left(\mathbf{x}^{\prime \prime}\right)>0$.

$48_{i+1}$ Pick an arbitrary $\mathbf{x}^{\prime \prime} \in U_{i+2}$. By $\left.45_{i+1}\right)$ we have $\Delta \rho_{i+1}\left(\mathbf{x}^{\prime \prime}\right)=0$, and by $\left(45_{i}\right)$, since $U_{i+2} \subset$ $U_{i+1}$, we have $\Delta f_{i}\left(\mathbf{x}^{\prime \prime}\right)=0$. Thus,

$$
\Delta f_{i+1}\left(\mathbf{x}^{\prime \prime}\right)=\Delta \rho_{i+1}\left(\mathbf{x}^{\prime \prime}\right)+(\xi+1) \cdot \Delta f_{i}\left(\mathbf{x}^{\prime \prime}\right)=0+(\xi+1) \cdot 0=0 .
$$

This completes the proof.

EXAMPLE 5.32. We compute $f_{1}, f_{2}$ and $f_{3}$ for the weak $L R F \tau=\left\langle x_{1}, x_{2}-x_{1}, x_{3}\right\rangle$ of Example 5.26 So we have

$$
\rho_{1}\left(x_{1}, x_{2}, x_{3}\right)=x_{1}, \quad \rho_{2}\left(x_{1}, x_{2}, x_{3}\right)=x_{2}-x_{1}, \quad \rho_{3}\left(x_{1}, x_{2}, x_{3}\right)=x_{3} .
$$

We let $A_{i}^{\prime \prime} \mathbf{x}^{\prime \prime} \leq \mathbf{c}_{i}^{\prime \prime}$, for $1 \leq i \leq 3$, be the constraint representations of the transition polyhedra.

$\left(f_{1}\right)$ We set $f_{1}\left(x_{1}, x_{2}, x_{3}\right)=\rho_{1}\left(x_{1}, x_{2}, x_{3}\right)=x_{1}$, as in the base-case of the induction.

$\left(f_{2}\right)$ We have $\bar{U}_{2}=\mathcal{Q}_{2} \cup \mathcal{Q}_{3}$, thus we solve 5152 twice, once with $A_{2}^{\prime \prime} \mathbf{x}^{\prime \prime} \leq \mathbf{c}_{2}^{\prime \prime}$ and once with $A_{3}^{\prime \prime} \mathbf{x}^{\prime \prime} \leq \mathbf{c}_{3}^{\prime \prime}$. In both cases

$$
(\vec{a},-\vec{a})=(-1,1,0,1,-1,0), \quad(-\vec{b}, \vec{b})=(-1,0,0,1,0,0) .
$$

We get $\xi_{1}=0$ and $\xi_{2}=1$, and thus we take $\xi=1$. Then we define

$$
f_{2}\left(x_{1}, x_{2}, x_{3}\right)=\rho_{2}\left(x_{1}, x_{2}, x_{3}\right)+(\xi+1) \cdot f_{1}\left(x_{1}, x_{2}, x_{3}\right)=x_{2}+x_{1} .
$$

$\left(f_{3}\right)$ We have $\bar{U}_{3}=\mathcal{Q}_{3}$, thus we solve 5152 for $A_{3}^{\prime \prime} \mathbf{x}^{\prime \prime} \leq \mathbf{c}_{3}^{\prime \prime},(\vec{a},-\vec{a})=(0,0,1,0,0,-1)$ and $(-\vec{b}, \vec{b})=$ $(-1,1,0,1,-1,0)$. We get $\xi=0$, and thus

$$
f_{3}\left(x_{1}, x_{2}, x_{3}\right)=\rho_{3}\left(x_{1}, x_{2}, x_{3}\right)+(\xi+1) \cdot f_{2}\left(x_{1}, x_{2}, x_{3}\right)=x_{3}+x_{2}+x_{1} .
$$

Now we show how to use $f_{1}, \ldots, f_{d}$ of Lemma 5.31 in order to construct a $L L R F$ for $\mathcal{Q}_{1}, \ldots, \mathcal{Q}_{k}$. We first state an auxiliary definition.

Definition 5.33. For affine functions $\rho_{1}, \ldots, \rho_{j}: \mathbb{Q}^{n} \rightarrow \mathbb{Q}$, and positive constants $\delta_{1}, \ldots, \delta_{j}$, define $\mathbf{R}\left(\left\langle\rho_{1}, \ldots, \rho_{j}\right\rangle,\left\langle\delta_{1}, \ldots, \delta_{j}\right\rangle\right)$ to be the set of $\mathbf{x}^{\prime \prime} \in \mathbb{Q}^{2 n}$ for which there is an $1 \leq i \leq j$ satisfying $37 \sqrt[39]{ }$. We say that such transitions $\mathbf{x}^{\prime \prime}$ are ranked by $\left\langle\rho_{1}, \ldots, \rho_{j}\right\rangle$ (with $\delta_{1}, \ldots, \delta_{j}$ ), or, to name the position, that they are ranked by $\rho_{i}$ in $\mathbf{R}\left(\left\langle\rho_{1}, \ldots, \rho_{j}\right\rangle,\left\langle\delta_{1}, \ldots, \delta_{j}\right\rangle\right)$. 
In the next lemma we construct a $L L R F \tau_{\ell}$ that ranks all transitions of $\mathcal{Q}_{\ell}$, for each $1 \leq \ell \leq k$. Afterwards, we show how $\tau_{1}, \ldots, \tau_{k}$ are combined into a $L L R F \tau$ for $\mathcal{Q}_{1}, \ldots, \mathcal{Q}_{k}$.

LEMMA 5.34. Let $1 \leq d^{\prime} \leq d$ be the largest $d^{\prime}$ such that $U_{d^{\prime}} \cap \mathcal{Q}_{\ell} \neq \emptyset$ for a given $\mathcal{Q}_{\ell}$. Then, $\tau_{\ell}=\left\langle\rho_{1}^{\prime}, \ldots, \rho_{d^{\prime}}^{\prime}\right\rangle$, where $\rho_{i}^{\prime}=f_{i}+i-1$, is a LLRF for $\mathcal{Q}_{\ell}$.

Proof. For $1 \leq i \leq d^{\prime}$, let $X_{i}=U_{i} \cap \mathcal{Q}_{\ell}$. Note that $X_{1}, \ldots, X_{d^{\prime}}$ are closed polyhedra, $\mathcal{Q}_{\ell}=X_{1} \supseteq \ldots \supseteq$ $X_{d^{\prime}} \neq \emptyset$, and $X_{d^{\prime}} \cap U_{d^{\prime}+1}=\emptyset$. We find $\delta_{1}, \ldots, \delta_{d^{\prime}}$ such that

$$
\mathbf{R}\left(\left\langle\rho_{1}^{\prime}, \ldots, \rho_{d^{\prime}}^{\prime}\right\rangle,\left\langle\delta_{1}, \ldots, \delta_{d^{\prime}}\right\rangle\right) \supseteq X_{1}
$$

This implies the lemma's statement since $X_{1}=\mathcal{Q}_{\ell}$. The proof is by induction, where we start from $i=d^{\prime}$ and proceed backwards. In the $i$-th step we find $\delta_{i}$ such that

$$
R_{i} \stackrel{\text { def }}{=} \mathbf{R}\left(\left\langle f_{i}^{[i]}, \ldots, f_{d^{\prime}}^{[i]}\right\rangle,\left\langle i \cdot \delta_{i}, i \cdot \delta_{i+1}, \ldots, i \cdot \delta_{d^{\prime}}\right\rangle\right) \supseteq X_{i}
$$

where $f_{j}^{[i]}=f_{j}+j-i$. Then, for $i=1$ we get 56 . First note that $\Delta f_{j}^{\left[i_{1}\right]}=\Delta f_{j}^{\left[i_{2}\right]}=\Delta f_{j}$ for any $1 \leq i_{1}<i_{2} \leq d^{\prime}$, this relation is fundamental to our proof.

Base-case. We take $i=d^{\prime}$, then $f_{d^{\prime}}^{\left[d^{\prime}\right]}=f_{d^{\prime}}$ and thus $R_{d^{\prime}}=\mathbf{R}\left(\left\langle f_{d^{\prime}}\right\rangle,\left\langle d^{\prime} \cdot \delta_{d^{\prime}}\right\rangle\right)$. Since $X_{d^{\prime}} \subseteq U_{d^{\prime}}$ and $X_{d^{\prime}} \cap U_{d^{\prime}+1}=\emptyset$, then, for any $\mathbf{x}^{\prime \prime} \in X_{d^{\prime}}$, by 46 $46_{d^{\prime}}$ we have $f_{d^{\prime}}(\mathbf{x}) \geq 0$ and by (47 $7_{d^{\prime}}$ we have $\Delta f_{d^{\prime}}\left(\mathbf{x}^{\prime \prime}\right)>0$. Now since $X_{d^{\prime}}$ is a closed polyhedron and $\Delta f_{d^{\prime}}$ is positive over $X_{d^{\prime}}, \Delta f_{d^{\prime}}$ must have a minimum $\mu>0$ in $X_{d^{\prime}}$. Define $\delta_{d^{\prime}}=\frac{\mu}{d^{\prime}}$, then $\Delta f_{d^{\prime}}\left(\mathbf{x}^{\prime \prime}\right) \geq \mu=d^{\prime} \cdot \delta_{d^{\prime}}$. Thus, $X_{d^{\prime}} \subseteq R_{d^{\prime}}$.

Induction hypothesis. $\quad X_{i+1} \subseteq R_{i+1}$.

Induction step. We find a value for $\delta_{i}$, and show that $X_{i} \subseteq R_{i}$. Note that $R_{i}$ uses the same $\delta_{i+1}, \ldots, \delta_{d^{\prime}}$ as $R_{i+1}$. Define $C_{i}$ as the set of all points $\mathrm{x}^{\prime \prime} \in X_{i}$ for which one of the following holds

$$
\begin{aligned}
\forall i<j \leq d^{\prime} . \Delta f_{j}^{[i]}\left(\mathbf{x}^{\prime \prime}\right) \leq i \cdot \delta_{j}, \\
\quad \exists l \geq i .\left(\forall i<j<l . \Delta f_{j}^{[i]}\left(\mathbf{x}^{\prime \prime}\right) \leq i \cdot \delta_{j}\right) \wedge f_{l}^{[i]}(\mathbf{x}) \leq 0 .
\end{aligned}
$$

Note that $C_{i}$ can be written as a finite union of closed polyhedra. We first prove that each transition in $X_{i} \backslash C_{i}$ is already ranked by $f_{j}^{[i]}$, for some $i<j \leq d^{\prime}$, in $R_{i}$. Then we show that the transitions of $C_{i}$ are ranked by $f_{i}^{[i]}$ for some value $\delta_{i}$.

Pick an arbitrary transition $\mathbf{x}^{\prime \prime} \in X_{i} \backslash C_{i}$, we show that it is ranked by $f_{j}^{[i]}$ in $R_{i}$, for some $j>i$. To see this, note the following:

- Since $\mathbf{x}^{\prime \prime} \notin C_{i}$, it violates (58) and (59). To violate (58), there must be $i<j \leq d^{\prime}$ for which

$$
\Delta f_{j}^{[i]}\left(\mathbf{x}^{\prime \prime}\right)>i \cdot \delta_{j}
$$

Take minimal such $j$, then, for any $i<j^{\prime}<j$, we have $\Delta f_{j^{\prime}}^{[i]}\left(\mathbf{x}^{\prime \prime}\right) \leq i \cdot \delta_{j^{\prime}}$. This means that the first conjunct of (59) is not violated by $\mathrm{x}^{\prime \prime}$ for any $i<l \leq j$, and thus, to violate (59), the second conjunct $f_{l}^{[i]}(\mathbf{x}) \leq 0$ must be violated, that is:

$$
\forall i<l \leq j \cdot f_{l}^{[i]}(\mathbf{x})>0
$$


- Let $i<l \leq d^{\prime}$. Since $X_{l}=U_{l} \cap \mathcal{Q}_{\ell}$ is not empty, $\mathcal{Q}_{\ell} \subseteq \bar{U}_{l}$. This means that $\mathbf{x}^{\prime \prime} \in \bar{U}_{l}$, and thus by $\left(47_{l} \mid 48_{l}\right)$ we have

$$
\Delta f_{l}^{[i]}\left(\mathbf{x}^{\prime \prime}\right)=\Delta f_{l}\left(\mathbf{x}^{\prime \prime}\right) \geq 0 .
$$

Moreover, since $\mathbf{x}^{\prime \prime} \in X_{i} \subseteq U_{i}$,

$$
f_{i}^{[i]}(\mathbf{x})=f_{i}(\mathbf{x}) \geq 0
$$

by $46_{i}$.

Inequalities 6063 show that $\mathbf{x}^{\prime \prime}$ is ranked by $\Delta f_{j}^{[i]}$ in $R_{i}$.

Now we show that the transitions of $C_{i}$ are ranked by $f_{i}^{[i]}$ in $R_{i}$, for some $\delta_{i}$. If $C_{i}=\emptyset$ then we simply take $\delta_{i}=\delta_{i+1}$, and clearly $X_{i} \subseteq R_{i}$ (since the transitions of $X_{i} \backslash C_{i}$ are ranked as we have seen above independently from $\delta_{i}$ ). Assume $C_{i} \neq \emptyset$. We first claim that $C_{i} \cap X_{i+1}=\emptyset$. To see this, take $\mathbf{x}^{\prime \prime} \in X_{i+1}$, by the induction hypothesis we have $X_{i+1} \subseteq R_{i+1}$ and thus there must be $f_{j}^{[i+1]}$, for some $i<j \leq d^{\prime}$, that ranks $\mathbf{x}^{\prime \prime}$, thus:

- $\Delta f_{j}^{[i]}\left(\mathbf{x}^{\prime \prime}\right)=\Delta f_{j}^{[i+1]}\left(\mathbf{x}^{\prime \prime}\right) \geq(i+1) \cdot \delta_{j}>i \cdot \delta_{j}$, so 580 is violated;

- $f_{l}^{[i+1]}(\mathbf{x}) \geq 0$ for any $i<l \leq j$, and thus $f_{l}^{[i]}(\mathbf{x})=f_{l}^{[i+1]}(\mathbf{x})+1 \geq 1$. This means that (59) cannot be true for any $i<l \leq j$, it also cannot be true for any $j<l \leq d^{\prime}$ since $\Delta f_{j}^{[i]}\left(\mathbf{x}^{\prime \prime}\right)>i \cdot \delta_{j}$ as we have seen in the previous point.

Now since $C_{i} \cap X_{i+1}=\emptyset$ and $C_{i} \subseteq X_{i}$ we get $C_{i} \subseteq X_{i} \backslash X_{i+1}$. We also know that $X_{i} \backslash X_{i+1} \subseteq \bar{U}_{i} \backslash U_{i+1}$ by definition, and that by $\left(47_{i}\right)$ we have $\Delta f_{i}\left(\mathbf{x}^{\prime \prime}\right)>0$ throughout $\bar{U}_{i} \backslash U_{i+1}$. This means that $\Delta f_{i}\left(\mathbf{x}^{\prime \prime}\right)>0$ throughout $C_{i}$ as well. Now since $C_{i}$ is a finite union of closed polyhedra, $\Delta f_{i}\left(\mathbf{x}^{\prime \prime}\right)$ must have a minimum $\mu>0$. Define $\delta_{i}=\frac{\mu}{i}$ then $f_{i}^{[i]}\left(\mathbf{x}^{\prime \prime}\right)=f_{i}(\mathbf{x})^{\prime \prime} \geq \mu=i \cdot \frac{\mu}{i}$. Moreover, by $46_{i}$ we have $f_{i}(\mathbf{x}) \geq 0$ and thus $f_{i}^{[i]}(\mathbf{x})=f_{i}(\mathbf{x}) \geq 0$. This proves that $\mathbf{x}^{\prime \prime} \in C_{i}$ is ranked by $f_{i}^{[i]}$ in $R_{i}$.

LEMMA 5.35. $\tau=\left\langle\rho_{1}^{\prime}, \ldots, \rho_{d}^{\prime}\right\rangle$, where $\rho_{j}^{\prime}=f_{j}+j-1$, is a LLRF for $\mathcal{Q}_{1}, \ldots, \mathcal{Q}_{k}$. Moreover, it has a minimal dimension, at most $n$.

Proof. That $\tau$ is a $L L R F$ follows immediately from Lemma 5.34, because the transitions of each $\mathcal{Q}_{\ell}$ are ranked in $\tau_{\ell}$, and each $\tau_{\ell}$ is a prefix of $\tau$. The minimality of the dimension follows from that of the weak $L L R F$ : if there were a shorter $L L R F$, since every $L L R F$ is a weak $L L R F$, it would contradict Lemma 5.28 .

EXAMPLE 5.36. Consider again the weak $L L R F$ of Example 5.26 and $f_{1}=x_{1}, f_{2}=x_{2}+x_{1}$ and $f_{3}=x_{3}+x_{2}+x_{1}$ that we have computed in Example 5.32 The corresponding LLRF is $\tau=$ $\left\langle x_{1}, x_{1}+x_{2}+1, x_{1}+x_{2}+x_{3}+2\right\rangle$, with $\delta_{1}=1, \delta_{2}=\frac{1}{2}$ and $\delta_{3}=\frac{1}{3}$.

THEOREM 5.37. LexLinRF $(\mathbb{Q})$ is PTIME-decidable.

Proof. Procedure LLRFSYN, which has polynomial-time complexity by Lemma 5.14 is complete for the existence of a weak $L L R F$. If no weak $L L R F$ exists then no $L L R F$ exists either, and by Lemma 5.35 , if one exists then there is a $L L R F$. 
Note that if only termination is of interest, then there is no reason to actually perform the construction of Lemmas 5.31 and 5.34 it suffices to check the existence of a weak $L L R F$. Ranking functions are also used to bound the number of iterations of loops. LRFs clearly provide linear bounds, and LLRFs provide (typically) polynomial bounds when each component of the corresponding LLRF has an upper bound (usually a function of the input). In this context, to obtain an explicit upper bound, we may need to carry out the construction of Lemmas 5.31 and 5.34 . This can be done in polynomial time since computing $f_{1}, \ldots, f_{n}$ as in Lemma 5.31 only requires solving $k L P$ problems of bit-size polynomially bounded in the input bit-size.

As mentioned above, the bound deduced from a $L L R F$ is typically, at least approximately, the product of the bounds on the individual components, and hence a polynomial of degree at least $d^{\prime}$. In the next theorem we show that, in fact, for $S L C$ loops we can always find a piecewise linear bound (this observation applies whether one is interested in ranking all rational points or just integer ones). Note that Alias et al. 2010 proved that an $S L C$ loop has a $L L R F$ if and only if it has a $L R F$, and thus has a linear bound on the number of iterations. However, our definition of $L L R F$ captures some $S L C$ loops that do not have a $L R F$, as seen in Example 2.6.

THEOREM 5.38. Let $\mathcal{Q}$ be the transition polyhedron of an $S L C$ loop, $\left\langle\rho_{1}, \ldots, \rho_{d}\right\rangle$ a (weak) $L L R F$ inferred by Procedure LLRFSYN, and $\tau=\left\langle\rho_{1}^{\prime}, \ldots, \rho_{d^{\prime}}^{\prime}\right\rangle$ a LLRF as constructed in Lemma 5.34 with corresponding $\delta_{1}, \ldots, \delta_{d^{\prime}}$. Given an input $\mathbf{x} \in \mathbb{Q}^{n}$, let $j$ be the minimum $1 \leq j \leq d^{\prime}$ such that $\rho_{j}^{\prime}(\mathbf{x})<0$, or $j=d^{\prime}$ if no one exists, then $\sum_{i=1}^{j-1}\left(\left\lfloor\rho_{i}^{\prime}(\mathbf{x}) / \delta_{i}\right\rfloor+1\right)$ is an upper bound on the number of iterations of $\mathcal{Q}$ when starting from $\mathbf{x}$.

Proof. By Lemma 5.31, any $\mathbf{z}^{\prime \prime} \in \mathcal{Q}$ satisfies $\Delta \rho_{i}^{\prime}\left(\mathbf{z}^{\prime \prime}\right) \geq 0$; for any $1 \leq i \leq d^{\prime}$, which means that once the $i$-th component of $\tau$ become negative, it is then disabled and cannot rank any transition anymore (since it remains negative). In addition, when a transition is ranked by the $i$-th component, $\Delta \rho_{i}^{\prime}\left(\mathbf{x}^{\prime \prime}\right) \geq \delta_{i}$ which, together with the above argument, means that the $i$-th component of $\tau$ can rank at most $\left\lfloor\rho_{i}^{\prime}(\mathbf{x}) / \delta_{i}\right\rfloor+1$ transitions before it becomes negative. Now since every transition in the execution trace must be ranked by some component $\rho_{i}^{\prime}$ of $\tau$, and $i$ cannot be $\geq j$ since such components are disabled right from the beginning, we get the upper bound $\sum_{i=1}^{j-1}\left(\left\lfloor\rho_{i}^{\prime}(\mathbf{x}) / \delta_{i}\right\rfloor+1\right)$.

Remarks:

1. If we are only interested in an upper bound up to a constant factor, we can avoid the construction of Lemmas 5.31 and 5.34 because $\sum_{i=1}^{j-1}\left(\left\lfloor\rho_{i}^{\prime}(\mathbf{x}) / \delta_{i}\right\rfloor+1\right)$ is $O\left(\sum_{i=1}^{d} \max \left(0, \rho_{i}(\mathbf{x})\right)\right.$.

2. The theorem is easily extended to conclude that the piecewise linear bound is also valid for $M L C$ loops, when $\rho_{d}$ ranks at least one transition from each $\mathcal{Q}_{i}$, that is, $U_{d} \cap \mathcal{Q}_{i} \neq \emptyset$ for all $1 \leq k \leq d$.

\section{Prototype Implementation}

The different algorithms presented in this paper for synthesizing LRFs an LLRFs, both for the general cases and the special PTIME cases, have been implemented. Our tool, iRANKFinder, can be tried out via http://www.loopkiller.com/irankfinder. It receives as input an $M L C$ loop in constraint representation, and allows applying different algorithms for $\operatorname{LinRF}(\mathbb{Z}), \operatorname{LinRF}(\mathbb{Q}), \operatorname{LExLinRF}(\mathbb{Z})$, or $\operatorname{LEXLinRF}(\mathbb{Q})$. For LRFs, the implementation includes the algorithms of Lemmas 3.19 and 4.24 . By default it uses the second one since the first one relies on the generator representation of the transition polyhedron, which may take exponential time to compute. For LLRFs it uses Algorithm 1. 


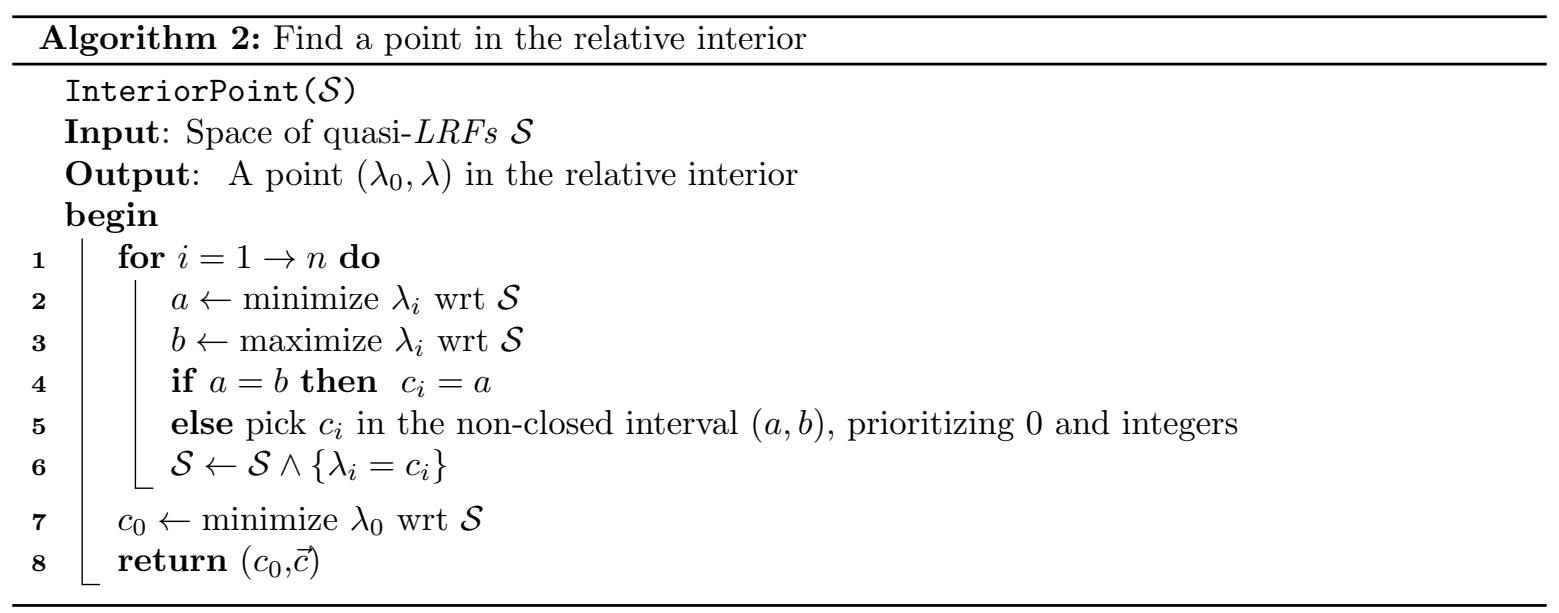

Our algorithm for synthesizing non-trivial quasi- $L R F s$, as described in Lemma 5.4 requires finding a point in the relative interior of a polyhedron $\mathcal{S}$. Note that $\mathcal{S}$ is of dimension $n^{\prime}=n+1+\sum_{i=1}^{k} 2 m_{i}$ and is defined by $m^{\prime}=k(8 n+2)+\sum_{i=1}^{k} 2 m_{i}$ inequalities, where $m_{i}$ is the number of inequalities in $\mathcal{Q}_{i}$. Existing algorithms for finding an interior point require solving at most $n^{\prime}$ or $m^{\prime} L P$ problems, and they have polynomial time complexity [Fukuda 2013, Sec. 8.3]. Now note that instead of finding a point in the relative interior of $\mathcal{S}$, we could also project $\mathcal{S}$ onto $\vec{\lambda}$, and then find a point in the relative interior of the resulting polyhedron $\mathcal{S}_{\mid \vec{\lambda}}$. It is easy to see that Lemma 5.4 remains valid. In our implementation we find such point without actually computing $\mathcal{S}_{\mid \vec{\lambda}}$, by solving only $2 n+1 L P$ problems. The underlying procedure is depicted in Algorithm 2 , it finds values for $\vec{\lambda}$ iteratively as follows: in the $i$-th iteration it computes the minimum and maximum values of $\lambda_{i}$ in $\mathcal{S}$, and then sets $\lambda_{i}$ to a value that lies between those extremes. Once all $\lambda_{i}$ are computed, we look for the minimum compatible value of $\lambda_{0}$, and then $\left(c_{0}, \vec{c}\right)$ is the desired point. We do not claim that the complexity of this algorithm is polynomial, since we add $\lambda_{i}=c$ to $\mathcal{S}$ in each iteration and thus the bit-size might grow exponentially. However, we have experimentally observed that it performs far better than an algorithm that finds a point in the relative interior of $\mathcal{S}$. Note that at Line 5 , we prioritize 0 over any other coefficient, as a heuristic to obtain "small" ranking functions. Moreover, we prioritize integer over fractional coefficients. Both measures are intended to get more readable results, but we think they may also improve time bounds inferred from our ranking functions.

Computing the integer hull of a polyhedron, in the case of $\operatorname{LinRF}(\mathbb{Z})$ and $\operatorname{LEx} \operatorname{LinRF}(\mathbb{Z})$, is done by first decomposing its set of inequalities into independent components, and then computing the integer hull of each component separately. Each set of inequalities is first matched against the PTIME cases of sections 4.1 and 4.2 . If this matching fails, the integer hull is computed using the algorithm of Hartmann 1988 as explained by Charles et al. 2009. Note that this algorithm supports only bounded polyhedra, the integer hull of an unbounded polyhedron is computed by considering a corresponding bounded one [Schrijver 1986, Th. 16.1, p. 231]. In addition, for octagonal relations, it gives the possibility of computing the tight closure instead of the integer hull. As we have seen in Section 4.3, when this option is used, completeness of $\operatorname{LinRF}(\mathbb{Z})$ is not guaranteed.

The Parma Polyhedra Library Bagnara et al. 2008b is used for converting between generator and constraints representations, solving (mixed) $L P$ problems, etc. 


\section{Related work}

There are several works Alias et al. 2010, Colón and Sipma 2001, Mesnard and Serebrenik 2008; Podelski and Rybalchenko 2004 Sohn and Gelder $1991 \mid$ that directly address the LINRF( $\mathbb{Q}$ ) problem for $S L C$ or $M L C$ loops. In all these works, the underlying techniques allow synthesizing $L R F s$ and not only deciding if one exists. The common observation to all these works is that synthesising $L R F s$ can be done by inferring the implied inequalities of a given polyhedron (the transition polyhedron of the loop), in particular inequalities like conditions (7) and (8) of Definition 2.3 that define a $L R F$. Regarding completeness, all these methods are complete for $\operatorname{LinRF}(\mathbb{Q})$ but not for $\operatorname{LinRF}(\mathbb{Z})$. They can also be used to approximate $\operatorname{LinRF}(\mathbb{Z})$ by relaxing the loop such that its variables range over $\mathbb{Q}$ instead of $\mathbb{Z}$, thus sacrificing completeness. All these methods have a corresponding PTIME algorithm. Exceptions in this line of research are the work of Bradley et al. 2005b and Cook et al. 2010] that directly address the $\operatorname{LinRF}(\mathbb{Z})$ problem for $M L C$ loops. Below, we comment in more detail on each of these works.

Sohn and Gelder 1991 considered $M L C$ loops with variables ranging over $\mathbb{N}$. These are abstractions of loops from logic programs. The loops were relaxed from $\mathbb{N}$ to $\mathbb{Q}_{+}$before seeking a $L R F$, however, this is not explicitly mentioned. The main observation in this work is that the duality theorem of $L P$ [Schrijver 1986, p. 92] can be used to infer inequalities that are implied by the transition polyhedron. The authors also mention that this was observed before by Lassez 1990 in the context of solving $\operatorname{CLP}(\mathbb{R})$ queries. Completeness was not addressed in this work, and the PTIME complexity was mentioned but not formally addressed. Later, Mesnard and Serebrenik 2008 formally proved that the techniques of Sohn and Gelder [1991] provide a complete PTIME method for $\operatorname{LinRF}(\mathbb{Q})$, also for the case of $M L C$ loops. They pointed out the incompleteness for $\operatorname{LinRF}(\mathbb{Z})$.

Probably the most popular work on the synthesis of LRFs is the one of Podelski and Rybalchenko 2004. They also observed the need for deriving inequalities implied by the transition polyhedron, but instead of using the duality theorem of $L P$ they used the affine form of Farkas' lemma [Schrijver 1986, p. 93]. Completeness was claimed, and the statement did not make it clear that the method is complete for $\operatorname{LinRF}(\mathbb{Q})$ but not for $\operatorname{LinRF}(\mathbb{Z})$. This was clarified, however, in the PhD thesis of Rybalchenko [2004]. One of the reasons for the impact of this work is its use in the Terminator tool [Cook et al. 2006], which demonstrated the use of LRFs in termination analysis of complex, real-world programs.

Bagnara et al. 2012 proved that the methods of Mesnard and Serebrenik 2008 and Podelski and Rybalchenko 2004 are actually equivalent, i.e., they compute the same set of $L R F s$. They also showed that the method of Podelski and Rybalchenko can, potentially, be more efficient since it requires solving rational constraints systems with fewer variables and constraints.

The earliest appearances of a solution based on Farkas' Lemma, that we know of, are by Colón and Sipma 2001, in the context of termination analysis, and by Feautrier 1992a, in the context of automatic parallelization of computations. Colón and Sipma 2001 did not claim that the problem can be solved in polynomial time, and indeed their implementation seems to have exponential complexity since they use generators and polars, despite the similarity of the underlying theory to that of Podelski and Rybalchenko 2004. Completeness was claimed, however it was not explicitly mentioned that the variables range over $\mathbb{Q}$ and not $\mathbb{Z}$ (the programs in the examples used integer variables). In this work the input loop comes with an initial condition on the input, which is used to infer a supporting invariant.

Feautrier 1992a described scheduling of computations that can be described by recursive equations. An abstraction to a form similar to an $M L C$ loop allowed him to compute a so-called schedule, which is essentially a ranking function, but used backwards, since the computations at the bottom of the 
recursion tree are to be completed first. Feautrier [1992b extends this work to lexicographic rankings; this work was subsequently extended by Alias et al. $[2010$ to $L L R F$ generation, as described below.

Cook et al. 2010 observed that the Farkas-lemma based solution is complete for $\operatorname{LiNRF}(\mathbb{Z})$ when the input $M L C$ loop is specified by integer polyhedra. They also mention that any polyhedron can be converted to an integer one, and that this might increase its size exponentially. Unlike our work, they do not address PTIME cases or the complexity of $\operatorname{LinRF}(\mathbb{Z})$. In fact, the main issue in that work is the synthesis of ranking functions for machine-level integers (bit-victors).

Bradley et al. 2005b directly addressed the $\operatorname{LinRF}(\mathbb{Z})$ problem for $M L C$ loops, and stated that the methods of Colón and Sipma [2001] and Podelski and Rybalchenko [2004] are not complete for $\operatorname{LinRF}(\mathbb{Z})$. Their technique is based on the observation that if there is a $L \overline{R F}$, then there exists one in which each coefficient $\lambda_{i}$ has a value in the interval $[-1,1]$, and moreover with denominators that are power of 2. Using this observation, they recursively search for the coefficients starting from a region defined by a hyper-rectangle in which each $\lambda_{i}$ is in the interval $[-1,1]$. Given a hyper-rectangle, the algorithm first checks if one of its corners defines a $L R F$, in which case it stops. Otherwise, the region is either pruned (if it can be verified that it contains no solution), or divided into smaller regions for recursive search. Testing if a region should be pruned is done by checking the satisfiability of a possibly exponential (in the number of variables) number of Presburger formulas. The algorithm will find a $L R F$ if exists, but it might not terminate if no $L R F$ exists. To make it practical, it is parametrized by the search depth, thus sacrificing completeness. It is interesting to note that the search-depth parameter in their algorithm actually bounds the bit-size of the ranking function coefficients. Our Corollary 3.22 shows that it is possible to deterministically bound this depth, that turns their algorithm into a complete one, though still exponential. In addition to $L R F s$, this technique is extended for inferring linear invariants over $\mathbb{Z}$.

The interest of Bradley et al. 2005b was in $M L C$ loops in which integer division by constants is allowed. It is incorrect to replace integer division $x^{\prime}=\frac{x}{c}$ by precise division, but the operation can be simulated by two paths of linear constraints: $\left\{x \geq 0, c \cdot x^{\prime}+y=x, 0 \leq y \leq c-1\right\}$ and $\left\{x \leq 0, c \cdot x^{\prime}-y=x, 0 \leq y \leq c-1\right\}$. This illustrates the usefulness of (multipath) linear-constraint loops.

Codish et al. 2005] studied the synthesis of LRFs for $S L C$ loops with size-change constraints (i.e., of the form $x_{i} \geq x_{j}^{\prime}+c$ where $c \in\{0,1\}$ ), and monotonicity constraints (i.e., of the form $X \geq Y+c$, where $X$ and $Y$ are variables or primed variables, and $c \in\{0,1\})$. In both cases the variables ranged over $\mathbb{N}$. For size-change constraints, they proved that the loop terminates if and only if a $L R F$ exists, moreover, such function has the form $\sum \lambda_{i} \cdot x_{i}$ with $\lambda_{i} \in\{0,1\}$. For the case of monotonicity constraints, they proved that the loop terminates if and only if a $L R F$ exists for the balanced version of the loop, and has the form $\sum \lambda_{i} \cdot x_{i}$ with $\lambda_{i} \in\{0, \pm 1\}$. Intuitively, a balanced loop includes the constraint $x_{i}^{\prime} \geq x_{j}^{\prime}+c$ if and only if it includes $x_{i} \geq x_{j}+c$. They showed how to balance the loop while preserving its termination behavior. Recently, Bozga et al. [2012] presented similar results for $S L C$ loops defined by octagonal relations, implying that termination is decidable (even PTIME) for such loops.

Cousot 2005 used Lagrangian relaxation for inferring possibly non-linear ranking functions. In the linear case, Lagrangian relaxation is similar to the affine form of Farkas' lemma.

The earliest work that we know, that addresses lexicographic-linear ranking functions, is that of Colón and Sipma 2002]. As in their previous work, they use LP methods based on the computation of polars. The $L L R F$ is not constructed explicitly but can be inferred from the results of the algorithm. Bradley et al. 2005a employed a constraint-solving approach to search for lexicographic-linear ranking functions, where a template solution is set up and linear programming is used to find the unknown 
coefficients in the template. Bradley et al. 2005c also relaxed the notion of ranking functions to functions that eventually decrease, while in another work Bradley et al. $2005 \mathrm{~d}$ they considered $M L C$ loops with polynomial transitions and the synthesis of lexicographic-polynomial ranking functions. All these works actually tackle an even more complex problem, since they also search for supporting invariants, based on the transition constraints and on given preconditions. Harris et al. 2011] demonstrate that it is advantageous, to a tool that is based on a CEGAR loop, to search for LLRFs instead of constructing transition invariants from LRFs only as in the original Terminator tool. They use a simplified version of the template method of Bradley et al. 2005a.

Alias et al. 2010 again extended the Farkas-lemma based solution for $\operatorname{LinRF}(\mathbb{Q})$ to the construction of LLRFs. Like Colón and Sipma 2002], they do it for programs with an arbitrary control-flow graph. Unlike the latter, they prove completeness of their procedure (which means completeness over the rationals), and their algorithm is of polynomial time. The goal of Alias et al. 2010 was to use these functions to derive cost bounds (like a bound on the worst-case number of transitions in terms of the initial state); this bound is (when it can be found) a polynomial, whose degree is at most the dimension of the (co-domain of the) lexicographic ranking function. Their construction produces a function of minimum dimension (within their class of ranking functions, which is narrower than ours, as discussed in Section 2p.

Decidability and complexity of termination (in general, not necessarily with LRFs or LLRFs) of $S L C$ and $M L C$ loops has been intensively studied for different classes of constraints. For $S L C$ loops, Tiwari 2004] proved that the problem is decidable when the update is linear and the variables range over $\mathbb{R}$. Braverman 2006 proved that this holds also for $\mathbb{Q}$, and for the homogeneous case it holds for $\mathbb{Z}$. Both considered universal termination, i.e., for all input. Also, in both cases they allow the use of strict inequalities in the condition. Ben-Amram et al. [2012] showed that the termination of $S L C$ loops is undecidable if the use of a single irrational coefficient is allowed, as well as for $M L C$ loops with at least two paths, and certain other variants.

For some specific forms of integer $M L C$ loops termination is decidable: Extending previous work on Size-Change Termination Lee et al. 2001, Ben-Amram 2011 proved that termination is decidable (more precisely: PSPACE-complete) for $M L C$ loops with monotonicity constraints (as defined above). Bozzelli and Pinchinat 2012 further extended the result (still PSPACE-complete) for Gap Constraints, which are constraints of the form $X-Y \geq c$ where $c \in \mathbb{N}$ and $X$ and $Y$ are variables or primed variables. This is, clearly, an extension of monotonicity constraints, which in particular allows for more precise representation of relations of variables to constants. Ben-Amram 2008 proved that for difference constraints over the integers, specifically updates of the form $x_{i}-x_{j}^{\prime} \geq c$ where $c \in \mathbb{Z}$, and guards $x_{i} \geq 0$, the termination problem becomes undecidable. However for a subclass in which each target (primed) variable might be constrained only once (in each path of a multiple-path loop) the problem is PSPACE-complete.

Regarding ranking functions, Ben-Amram 2011] shows that every terminating program of the considered form has a ranking function which is piecewise lexicographic. This is achieved by transforming the program (by splitting CFG nodes) into one that is guaranteed to have a $L L R F$. Such a result is probably achievable for the gap constraints of Bozzelli and Pinchinat 2012 as well. However, it is unknown how to explicitly construct ranking functions for difference constraints as those used by Ben-Amram 2008. 


\section{Concluding Remarks}

We have studied the Linear Ranking problem for $S L C$ and $M L C$ linear-constraint loops and observed the difference between the $\operatorname{LinRF}(\mathbb{Q})$ problem, where variables range over the rationals, and the $\operatorname{LinRF}(\mathbb{Z})$ problem, where variables only take integer values. In practice, the latter is more common, but the complexity of the problem has not been studied before; the common approach has been to relax the problem to the rationals, where complete, polynomial-time decision procedures have been known.

We have confirmed that $\operatorname{LinRF}(\mathbb{Z})$ is a harder problem, proving it to be coNP-complete. On a positive note, this shows that there is a complete solution, even if exponential-time. We further showed that some special cases of importance do have a PTIME solution. The latter results arise from a proof that for integer polyhedra, $\operatorname{LinRF}(\mathbb{Z})$ and $\operatorname{LinRF}(\mathbb{Q})$ are equivalent. Interestingly, this is not the case for termination in general. For example, the transition polyhedron of the $S L C$ loop "while $x \geq 0$ do $x^{\prime}=10-2 x$ " is integral; the loop terminates when the variables range over $\mathbb{Z}$ but does not terminate when they range over $\mathbb{Q}$, specifically for $x=3 \frac{1}{3}$. Note that this loop does not have a $L R F$ over the integers.

We have obtained results similar to the above regarding the $\operatorname{LExLiNRF}(\mathbb{Z})$ problem, the existence of lexicographic-linear ranking functions. Our polynomial-time algorithm for $\operatorname{LExLinRF}(\mathbb{Q})$ is also new, and extends the class of functions that can be found by the previously known polynomial-time algorithm of Alias et al. 2010. Our algorithm is optimal, in the sense that it synthesizes LLRFs with minimal dimension.

A more general notion of ranking function applies to an arbitrary control-flow graph with transitions specified by source and target nodes as well as linear constraints on the values of variables. In this setting, one seeks to associate a (possibly different) lexicographic-linear (or linear) function $\tau_{\nu}$ with each node $\nu$, so that on a transition from $\nu$ to $\nu^{\prime}$ we should have $\tau_{\nu}(\mathbf{x}) \succ_{\text {lex }} \tau_{\nu^{\prime}}\left(\mathbf{x}^{\prime}\right)$. Such functions can be found by $L P$, a procedure complete over the rationals, using a simple extension of the solution for the loops we have discussed [Alias et al. 2010, Mesnard and Serebrenik 2008. The considerations regarding the complexity of the corresponding problems over integers are essentially the same as those we have presented, and we preferred to use the simpler model for clearer presentation.

In all examples that we have discussed in this paper, when a loop has a $L R F$ over $\mathbb{Z}$ but not over $\mathbb{Q}$, then the loop did not terminate over $\mathbb{Q}$. This is, however, not the case in general. A counter-example can be constructed by combining (i.e., executing simultaneously) the loop of Example 3.6 and Loop (1) of Section 1 .

In the context of complexity (cost) analysis, there is a special interest in LRFs that decrease at least by 1 in each iteration, since they bound the number of iterations of a given loop. In order to get tight bounds, even if $\mathcal{Q}$ has a $L R F$ it might be worthwhile to compute one for $I(\mathcal{Q})$. To see this, let us add $4 x_{1} \geq 3$ to the condition of Loop (1) in Section 1 . Then, both $\mathcal{Q}$ and $I(\mathcal{Q})$ have LRFs. For $I(\mathcal{Q})$ the most tight one (under the requirement to decrease by at least 1 ) is $f_{1}\left(x_{1}, x_{2}\right)=x_{1}+x_{2}-1$, while for $\mathcal{Q}$ it is $f_{2}\left(x_{1}, x_{2}\right)=2 x_{1}+2 x_{2}-2$. Hence, a better bound is obtained using $I(\mathcal{Q})$. The same observation applies to loop parallelization: the functions' value gives the schedule's latency (depth of the computation tree) and a lower value is preferable. 


\section{References}

Elvira Albert, Puri Arenas, Samir Genaim, German Puebla, and Damiano Zanardini. Costa: Design and implementation of a cost and termination analyzer for java bytecode. In Frank S. de Boer, Marcello M. Bonsangue, Susanne Graf, and Willem P. de Roever, editors, Formal Methods for Components and Objects, FMCO'0\%, volume 5382 of LNCS, pages 113-132. Springer, 2007.

Elvira Albert, Puri Arenas, Samir Genaim, and Germán Puebla. Closed-form upper bounds in static cost analysis. J. Autom. Reasoning, 46(2):161-203, 2011.

Christophe Alias, Alain Darte, Paul Feautrier, and Laure Gonnord. Multi-dimensional rankings, program termination, and complexity bounds of flowchart programs. In Radhia Cousot and Matthieu Martel, editors, Static Analysis Symposium, SAS'10, volume 6337 of LNCS, pages 117-133. Springer, 2010 .

Roberto Bagnara, Patricia M. Hill, and Enea Zaffanella. An improved tight closure algorithm for integer octagonal constraints. In Francesco Logozzo, Doron Peled, and Lenore D. Zuck, editors, Verification, Model Checking, and Abstract Interpretation, VMCAI'08, volume 4905 of LNCS, pages 8-21. Springer, 2008a.

Roberto Bagnara, Patricia M. Hill, and Enea Zaffanella. The parma polyhedra library: Toward a complete set of numerical abstractions for the analysis and verification of hardware and software systems. Sci. Comput. Program., 72(1-2):3-21, 2008b.

Roberto Bagnara, Fred Mesnard, Andrea Pescetti, and Enea Zaffanella. A new look at the automatic synthesis of linear ranking functions. Inf. Comput., 215:47-67, 2012.

Amir M. Ben-Amram. Size-change termination with difference constraints. ACM Trans. Program. Lang. Syst., 30(3), 2008.

Amir M. Ben-Amram. Monotonicity constraints for termination in the integer domain. Logical Methods in Computer Science, 7(3), 2011.

Amir M. Ben-Amram and Samir Genaim. On the linear ranking problem for integer linear-constraint loops. In Proceedings of the 40th annual ACM SIGPLAN-SIGACT symposium on Principles of programming languages, POPL '13, pages 51-62, New York, NY, USA, 2013. ACM. doi: 10.1145/ 2429069.2429078 .

Amir M. Ben-Amram, Samir Genaim, and Abu Naser Masud. On the termination of integer loops. ACM Trans. Program. Lang. Syst., 34(4):16:1-16:24, December 2012. ISSN 0164-0925. doi: 10. $1145 / 2400676.2400679$.

Marius Bozga, Radu Iosif, and Filip Konecný. Deciding conditional termination. In Cormac Flanagan and Barbara König, editors, Tools and Algorithms for the Construction and Analysis of Systems, TACAS'12, volume 7214 of LNCS, pages 252-266. Springer, 2012.

Laura Bozzelli and Sophie Pinchinat. Verification of gap-order constraint abstractions of counter systems. In Viktor Kuncak and Andrey Rybalchenko, editors, Verification, Model Checking, and Abstract Interpretation, VMCAI'12, volume 7148 of LNCS, pages 88-103. Springer, 2012. 
Aaron R. Bradley, Zohar Manna, and Henny B. Sipma. Linear ranking with reachability. In Kousha Etessami and Sriram K. Rajamani, editors, Computer Aided Verification, CAV'05, volume 3576 of LNCS, pages 491-504. Springer, 2005a.

Aaron R. Bradley, Zohar Manna, and Henny B. Sipma. Termination analysis of integer linear loops. In Martín Abadi and Luca de Alfaro, editors, Concurrency Theory, CONCUR 2005, volume 3653 of $L N C S$, pages $488-502$. Springer, 2005b.

Aaron R. Bradley, Zohar Manna, and Henny B. Sipma. The polyranking principle. In Luís Caires, Giuseppe F. Italiano, Luís Monteiro, Catuscia Palamidessi, and Moti Yung, editors, International Colloquium on Automata, Languages and Programming, ICALP'05, volume 3580 of LNCS, pages 1349-1361. Springer, 2005c.

Aaron R. Bradley, Zohar Manna, and Henny B. Sipma. Termination of polynomial programs. In Radhia Cousot, editor, Verification, Model Checking, and Abstract Interpretation, VMCAI'05, volume 3385 of $L N C S$, pages 113-129. Springer, 2005d.

Mark Braverman. Termination of integer linear programs. In Thomas Ball and Robert B. Jones, editors, Computer Aided Verification, CAV'06, volume 4144 of LNCS, pages 372-385. Springer, 2006 .

Maurice Bruynooghe, Michael Codish, John P. Gallagher, Samir Genaim, and Wim Vanhoof. Termination analysis of logic programs through combination of type-based norms. ACM Trans. Program. Lang. Syst., 29(2), 2007.

Philip J. Charles, Jacob M. Howe, and Andy King. Integer polyhedra for program analysis. In Andrew V. Goldberg and Yunhong Zhou, editors, Algorithmic Aspects in Information and Management, AAIM'09, volume 5564 of LNCS, pages 85-99. Springer, 2009.

Michael Codish, Vitaly Lagoon, and Peter J. Stuckey. Testing for termination with monotonicity constraints. In Maurizio Gabbrielli and Gopal Gupta, editors, International Conference on Logic Programming, ICLP'05, volume 3668 of LNCS, pages 326-340. Springer, 2005.

Michael Colón and Henny Sipma. Synthesis of linear ranking functions. In Tiziana Margaria and Wang Yi, editors, Tools and Algorithms for the Construction and Analysis of Systems, TACAS'01, volume 2031 of $L N C S$, pages 67-81. Springer, 2001.

Michael Colón and Henny Sipma. Practical methods for proving program termination. In Ed Brinksma and Kim Guldstrand Larsen, editors, Computer Aided Verification, 14th International Conference, CAV'02, Copenhagen, Denmark, July 27-31, 2002, Proceedings, volume 2404 of LNCS, pages 442454. Springer, 2002.

Byron Cook, Andreas Podelski, and Andrey Rybalchenko. Termination proofs for systems code. In Michael I. Schwartzbach and Thomas Ball, editors, Programming Language Design and Implementation, PLDI'06, pages 415-426. ACM, 2006.

Byron Cook, Daniel Kroening, Philipp Rümmer, and Christoph M. Wintersteiger. Ranking function synthesis for bit-vector relations. In Javier Esparza and Rupak Majumdar, editors, Tools and Algorithms for the Construction and Analysis of Systems, 16th International Conference, TACAS'10, volume 6015 of $L N C S$, pages 236-250. Springer, 2010. 
Patrick Cousot. Proving program invariance and termination by parametric abstraction, lagrangian relaxation and semidefinite programming. In Radhia Cousot, editor, Verification, Model Checking, and Abstract Interpretation, VMCAI'05, volume 3385 of LNCS, pages 1-24, 2005.

Alain Darte. Understanding loops: The influence of the decomposition of Karp, Miller, and Winograd. In Formal Methods and Models for Codesign, MEMOCODE'10, pages 139-148. IEEE Computer Society, 2010.

Paul Feautrier. Some efficient solutions to the affine scheduling problem. I. one-dimensional time. International Journal of Parallel Programming, 21(5):313-347, 1992a.

Paul Feautrier. Some efficient solutions to the affine scheduling problem. II. multidimensional time. International Journal of Parallel Programming, 21(6):389-420, 1992b.

Komei Fukuda. Lecture: Polyhedral computation, spring 2013. Available at http://wwwoldurls.inf.ethz.ch/personal/fukudak/lect/pclect/notes2013, February 2013.

Michael R. Garey and David S. Johnson. Computers and Intractability. W.H. Freeman and Co., New York, 1979.

Jürgen Giesl, René Thiemann, Peter Schneider-Kamp, and Stephan Falke. Automated termination proofs with aprove. In Vincent van Oostrom, editor, Rewriting Techniques and Applications, RTA'04, volume 3091 of $L N C S$, pages 210-220. Springer, 2004.

William R Harris, Akash Lal, Aditya V Nori, and Sriram K Rajamani. Alternation for termination. In Static Analysis Symposium, SAS 2011, volume 6337 of LNCS, pages 304-319. Springer, 2011.

Mark E. Hartmann. Cutting Planes and the Complexity of the Integer Hull. PhD thesis, School of Operations Research and Industrial Engineering, Cornell University, 1988.

Warwick Harvey. Computing two-dimensional integer hulls. SIAM J. Comput., 28(6):2285-2299, 1999.

Warwick Harvey and Peter J. Stuckey. A unit two variable per inequality integer constraint solver for constraint logic programming. In Australasian Computer Science Conference, ACSC'97, pages 102-111, 1997.

R. M. Karp. Reducibility among combinatorial problems. In R. E. Miller and J. W. Thatcher, editors, Complexity of Computer Computations, pages 85-103. Plenum Press, New York, 1972.

Richard M. Karp and Christos H. Papadimitriou. On linear characterizations of combinatorial optimization problems. In Symp. on Foundations of Computer Science, FOCS'80, pages 1-9. IEEE Computer Society, 1980.

Daniel Kroening, Natasha Sharygina, Aliaksei Tsitovich, and Christoph Wintersteiger. Termination analysis with compositional transition invariants. In Computer Aided Verification, CAV 2010, volume 6174 of $L N C S$, pages 89-103. Springer, 2010.

Jean-Louis Lassez. Querying constraints. In Symposium on Principles of Database Systems, pages 288-298. ACM Press, 1990. 
Chin Soon Lee, Neil D. Jones, and Amir M. Ben-Amram. The size-change principle for program termination. In Chris Hankin and Dave Schmidt, editors, Symposium on Principles of Programming Languages, POPL'01, pages 81-92, 2001.

Naomi Lindenstrauss and Yehoshua Sagiv. Automatic termination analysis of Prolog programs. In Lee Naish, editor, International Conference on Logic Programming, ICLP'97, pages 64-77. MIT Press, 1997.

Frédéric Mesnard and Alexander Serebrenik. Recurrence with affine level mappings is p-time decidable for $\operatorname{clp}(\mathrm{r})$. TPLP, 8(1):111-119, 2008.

Antoine Miné. The octagon abstract domain. Higher-Order and Symbolic Computation, 19(1):31-100, March 2006.

Andreas Podelski and Andrey Rybalchenko. A complete method for the synthesis of linear ranking functions. In Bernhard Steffen and Giorgio Levi, editors, Verification, Model Checking, and Abstract Interpretation, VMCAI'04, volume 2937 of LNCS, pages 239-251. Springer, 2004.

Peter Z. Revesz. Tightened transitive closure of integer addition constraints. In Vadim Bulitko and J. Christopher Beck, editors, Symposium on Abstraction, Reformulation, and Approximation, SARA'09, 2009.

Andrey Rybalchenko. Temporal Verification with Transition Invariants. PhD thesis, Universität des Saarlandes, 2004.

Alexander Schrijver. Theory of Linear and Integer Programming. John Wiley and Sons, New York, 1986.

Kirack Sohn and Allen Van Gelder. Termination detection in logic programs using argument sizes. In Daniel J. Rosenkrantz, editor, Symposium on Principles of Database Systems, pages 216-226. ACM Press, 1991.

Fausto Spoto, Fred Mesnard, and Étienne Payet. A termination analyzer for java bytecode based on path-length. ACM Trans. Program. Lang. Syst., 32(3), 2010.

Éva Tardos. A strongly polynomial algorithm to solve combinatorial linear programs. Operations Research, 34:250-256, 1986.

Ashish Tiwari. Termination of linear programs. In Rajeev Alur and Doron Peled, editors, Computer Aided Verification, CAV'04, volume 3114 of LNCS, pages 387-390. Springer, 2004. 